\title{
Quantum collision theory with phase-space distributions
}

\author{
P. Carruthers
}

Theoretical Division, Los Alamos National Laboratory, Los Alamos, New Mexico 87545

\section{F. Zachariasen *}

California Institute of Technology, Pasadena, California 91125

Quantum-mechanical phase-space distributions, introduced by Wigner in 1932, provide an intuitive alternative to the usual wave-function approach to problems in scattering and reaction theory. The aim of the present work is to collect and extend previous efforts in a unified way, emphasizing the parallels among problems in ordinary quantum theory, nuclear physics, chemical physics, and quantum field theory. The method is especially useful in providing easy reductions to classical physics and kinetic regimes under suitable conditions. Section II, dealing in detail with potential scattering of a spinless nonrelativistic particle, provides the background for more complex problems. Following a brief description of the two-body problem, the authors address the $N$-body problem with special attention to hierarchy closures, BoltzmannVlasov equations, and hydrodynamic aspects. The final section sketches past and possibly future applications to a wide variety of problems.

\section{CONTENTS}

I. Introduction

II. Nonrelativistic Potential Scattering
A. Definitions
E. The eikonal approximation
F. The bound-state problem
G. Hydrodynamic aspects
H. Effect of an external potential
I. Gauge invariance
J. Spin variables

B. Equation of motion: relation to the classical limit

C. Integral form of the scattering equations

D. Exact formula for the scattering cross section

III. The Nonrelativistic Two-body Problem
A. Definitions
B. Equation of motion
C. Technical interlude: free $N$-particle retarded Green's functions
D. Equations of motion in the $(p, q)$ basis
E. Boundary conditions

IV. The $N$-body Problem: Hierarchies and the BoltzmannVlasov Equation

A. Definitions and equation of motion

B. Coupled hierarchy equations for reduced distribution functions

C. Truncation schemes and the Boltzmann-Vlasov equation

D. Hydrodynamic equations

E. Formulation in second quantization

F. Effect of spin and statistics

V. Possible Applications in Interactions of Composite Systems

A. Composite particle scattering in the Liouville formalism

B. Models of nucleus-nucleus collisions

C. Atomic and molecular collisions

D. Solid-state physics

E. Second-quantization approach to inclusive reactions

F. Relativistic formulation of the phase-space approach to scattering and production processes

"On leave of absence: Los Alamos National Laboratory, Los Alamos, New Mexico 87545.
Acknowledgments

References

283

283

\section{INTRODUCTION}

In recent years one can discern an increasing interest in the use of quantum-mechanical phase-space distributions for the formulation and solution of scattering and production problems. These distributions, which are quantum generalizations of Boltzmann's $N$-particle distribution $f_{N}\left(p_{1} R_{1}, \ldots, p_{N} R_{N}, t\right)$, provide an intuitive picture of complex collision processes.

As is well known (Tolman, 1938), the $6 N$ dimensional phase space of the position coordinates $q_{i}$ and momenta $p_{i}$ provides a useful arena for the description of classical mechanics. Quantum mechanics, which forbids states having simultaneously definite $p_{i}$ and $q_{i}$, requires the replacement of the phase-space distribution function by the density "matrix" (von Neumann, 1955). An attractive version of the density matrix discovered by Szilard and Wigner (Wigner, 1932) allows the expression of quantum dynamics in a form directly comparable with the classical analog. The authors' interest in this approach occurred when they noticed that the field-theoretic formulation of inclusive production process involved second-quantized analogs of the Wigner distribution function (Carruthers and Zachariasen, 1974, 1976). In this formulation the $N$ particle covariant distribution functions are directly connected with the inclusive differential cross sections. Because of the mathematical complexities of the relativistic field-theory problem, no useful predictions have yet come out of this formalism. In nuclear physics, Remler and collaborators initiated and developed a program in which nuclear reaction theory is formulated in the language of the Wigner distribution function (Remler, 1975, 1981; Remler and Sathe, 1975, 1978). Meanwhile, phase-space methods (sometimes classical) were being developed by quantum chemists in order to elucidate chemical reaction problems (Brown and Heller, 1982; Eu, 1971, 1975; Heller, 1976, 1977; Lee and Scully, 1980; Miles and Dahler, 1970). We hope that the present work will encourage 
communication among these disciplines and will lead to the recognition of the phase-space method as one of universal utility.

Over the years, several authors noted the possibility of expressing potential scattering cross sections in terms of Wigner functions (Irving and Zwanzig, 1951; Ross and Kirkwood, 1954; Mori, Oppenheim, and Ross, 1962; Huguenin, 1973; Prugovečki, 1978a). However, the principal interest in these years was the derivation of quantum-kinetic or Boltzmann equations for uniform or almost uniform extended systems. Some other works concerned with the Boltzmann equation are Uehling and Uhlenbeck (1933); Mori and Ross (1958); Snider (1960); Snider and Sanctuary (1971); Prugovečki (1978b). The generalization of this approach to nonrelativistic field theory and the modern formulation of the many-body problem is explained in the book by Kadanoff and Baym (1962). The most important qualitative consequence of the modern formulation is the natural manner in which "self-consistent field" theories appear in the simplest approximation. According to the nature of the system and the statistics of the particles, these approximations carry the names of Vlasov, time-dependent Hartree-Fock (TDHF), and random-phase approximation. Secondly, the transport equation can be obtained by suitable truncation of the coupled equations of motion. These techniques can be carried over to relativistic matter. One of the earliest such works, using Green's-function techniques, is that of Bezzerides and DuBois (1972). The reader is referred to the review of Hakim (1978) and the thesis of de Boer $(1979,1980)$ for further information and references on this subject.

The relation of transport equations to classical or quantum dynamics, as expressed through the coupled hierarchy of distribution functions, continues to be a problem of fundamental interest (Cohen, 1968). In this paper we shall indicate how such equations can (under suitable conditions) describe a portion of a collision process involving many particles.

Another fundamental line of investigation concerns the representation of quantum mechanics by phase-space distributions. Among the works on this topic, we mention the following: Groenewald (1946); Moyal (1949); Baker (1958); Barut (1957); Imre, Özizmir, Rosenbaum, and Zweifel (1967); Brittin (1971); Wigner (1971); Ali and Prugovečki (1977a, 1977b); Grossman and Huguenin (1978); Prugovečki (1978c); Balazs (1980); O'Connell and Wigner (1981a, 1981b). A review of the formalism of the Wigner function and its relation to the Weyl transformation (Weyl, 1927) has been given by de Groot and Suttorp (1972). A related topic in this area of research is the analysis of alternative definitions of distribution functions designed to satisfy special criteria (Baker, 1958; Glauber, 1963; Cohen, 1966; Agarwal and Wolf, 1968; Lax, 1968). For our purposes we do not need to worry about these fine points. In the description of inclusive scattering processes, the Wigner function arises naturally (especially in second quantization). Cross sections are directly related to it, and other issues are of secondary concern.
Recently the increased activity in heavy-ion physics has led to greater interest in phase-space distributions. Currently popular models (intranuclear cascade model, "hot spot" model, hydrodynamical models) which emphasize collective and transport behavior are especially suitable for examination in this framework. It was, in fact, our earlier interest in the Landau hydrodynamical model (Landau, 1953) of particle production which led to our search for an underlying kinetic formulation of that theory. We hope that the present work will lay the foundation for a proper description of nucleon-nucleus and nucleus-nucleus collisions. We also remark that pionnucleus scattering fits conveniently into the field-theoretic version of our formalism, with a proper (non-wavefunction) description of the pion field. References to these developments are given in Sec. VI.

The aim of the present review is to formulate in phasespace distribution language the nonrelativistic $N$-particle scattering of particles interacting via two-body potentials. Technical complications introduced by spin, statistics, and other degrees of freedom (e.g., isospin) will usually be suppressed. The work should be regarded as a personal essay. It is partly review and partly original, and is mostly taken from our notebooks and lecture notes dating back several years. Points of contact will be found with many of the references, however. Our main hope is to explain the lucid physical meaning of this approach to collision theory and to carry the development far enough to inspire realistic calculations. We always keep in sight the properties special to this formalism, namely, the description of collective modes and the transport and hydrodynamic behavior. Unfortunately, many of the applications will require extensive numerical work. However, that is the nature of the problems of principal interest.

\section{NONRELATIVISTIC POTENTIAL SCATTERING}

\section{A. Definitions}

We consider in some detail the scattering of a nonrelativistic, spinless, particle from a static potential well $V$. Since we are ultimately interested in the detailed spacetime evolution of complicated systems, we use wave packets throughout. The usual results can be obtained by taking suitable limits.

As is well known, one has to give up the concept of particle trajectories in quantum theory. The density of particles is expressed in terms of the wave function by

$$
n(\underline{R}, t)=|\psi(\underline{R}, t)|^{2} .
$$

Writing $\psi$ as

$$
\psi(\underline{R}, t)=\int \frac{d^{3} p}{(2 \pi)^{3 / 2}} C(\underline{p}, t) e^{i \underline{p} \cdot \underline{R}},
$$

we see that the momentum distribution implied by $\psi$ is

$$
n(\underline{p}, t)=|C(\underline{p}, t)|^{2} .
$$

One cannot specify simultaneously the position and 
momentum better than indicated by Heisenberg's uncertainty principle:

$$
\Delta x \Delta p \geq \frac{1}{2} .
$$

(Planck's constant $\hbar$ is set equal to unity except when necessary to clarify physical interpretations.)

The demise of the orbit also casts a pall on the traditional formulation of statistical mechanics in terms of distribution functions defined on phase space. The simplest of these, the one-particle function $f(p, R, t)$, immortalized by Boltzmann, is defined so that $f(p, R, t) d^{3} R d^{3} p$ is the probability of finding a particle of momentum $p$ and position $R$ in the phase-space volume $d^{3} R d^{3} p$. The coordinate and momentum densities are, correspondingly,

$$
\begin{aligned}
& n(\underline{R}, t)=\int d^{3} p f(\underline{p}, \underline{R}, t), \\
& n(\underline{p}, t)=\int d^{3} R f(\underline{p}, \underline{R}, t),
\end{aligned}
$$

with the overall normalization taken as unity. Note that the $f$ suitable to a single classical particle in a pure state,

$$
f(\underline{p}, \underline{R}, t)=\delta(\underline{R}-\underline{R}(t)) \delta(p-\underline{p}(t))
$$

where $R(t)$ defines the classical orbit and $p(t)=m \dot{R}(t)$, is in direct conflict with the uncertainty principle.

Wigner (1932) has proposed a construction which both respects the rules of quantum mechanics and recaptures most of the desired features of the Boltzmann function. Notice that the density matrix (for a pure state)

$$
\psi^{*}\left(x_{1}, t\right) \psi\left(\underline{x}_{2}, t\right)
$$

has six $c$-number variables besides $t$. Introducing relative and center-of-mass coordinates by

$$
\begin{aligned}
& \underline{r}=\left(\underline{x}_{2}-\underline{x}_{1}\right), \\
& \underline{R}=\left(\underline{x}_{1}+\underline{x}_{2}\right) / 2,
\end{aligned}
$$

we obtain the Wigner function by making a Fourier transform on the relative coordinate:

$$
f(\underline{p}, \underline{R}, t)=\int \frac{d^{3} r}{(2 \pi)^{3}} e^{-\underline{i p} \cdot \underline{r}} \psi^{*}\left(\underline{R}-\frac{1}{2} \underline{r}, t\right) \psi\left(\underline{R}+\frac{1}{2} \underline{r}, t\right) .
$$

The variable $p$ is not an operator, but is simply a judiciously chosen ordinary vector. It is also useful to write $f$ in terms of the momentum basis

$$
f(\underline{p}, \underline{R}, t)=\int \frac{d^{3} q}{(2 \pi)^{3}} e^{i \underline{q} \cdot \underline{R}} C^{*}\left(\underline{p}-\frac{1}{2} \underline{q}, t\right) C\left(\underline{p}+\frac{1}{2} \underline{q}, t\right) .
$$

From these definitions we see that

$$
\begin{aligned}
& \int d^{3} p f(\underline{p}, \underline{R}, t)=|\psi(\underline{R}, t)|^{2}, \\
& \int d^{3} R f(\underline{p}, \underline{R}, t)=|C(\underline{p}, t)|^{2},
\end{aligned}
$$

in analogy to Eqs. (2.5) and (2.6).

The function $f$ is real, but not necessarily positive definite. The lack of assured positivity will not concern us unduly since we are mainly concerned with computation of positive definite asymptotic quantities like (2.13), which give the differential cross sections.

Since Eq. (2.10) is nothing but a particular representation of the density matrix, what we have is essentially the density-matrix formulation of quantum mechanics. Our approach is also closely connected with the Green'sfunction treatment of the many-body problem, as will become clear in Sec. V.E describing the second-quantization formulation of the problem. (In that section we shall consider more general density matrices.) Although the density-matrix description of quantum dynamics has little to offer in simplicity for easy problems involving only a few particles, it really comes into its own for complex, many-particle dynamics. We shall argue that the Wigner version, suitably generalized, is the most natural way to formulate and solve such problems.

The simplest example is a free particle,

$C(\underline{p}, t)=C(\underline{p}) \exp \left(-i p^{2} t / 2 m\right)$,

$f_{0}(\underline{p}, \underline{R}, t)=\int \frac{d^{3} q}{(2 \pi)^{3}} e^{+i q \cdot\left(\underline{R}-\underline{v}_{p} t\right)} C^{*}\left(\underline{p}-\frac{1}{2} q\right) C\left(\underline{p}+\frac{1}{2} q\right)$,

where $\underline{v}_{p}=p / m$. For a wave packet made up of free particles, $f_{0}$ depends on $\underline{R}$ as $\underline{R}-\underline{v}$. The example of the Gaussian momentum packet (peaked at $p_{0}$ and $R_{0}$ ) is especially useful and interesting,

$$
\begin{aligned}
C(\underline{p})=\frac{\exp \left[-\left(p-p_{0}\right)^{2} / 4 \sigma\right]}{(2 \pi \sigma) 3 / 4} \exp \left(-i \underline{p} \cdot \underline{R}_{0}\right), \\
f_{0}(\underline{p}, \underline{R}, t)=\frac{\exp \left[-\left(p-p_{0}\right)^{2} / 2 \sigma\right]}{(2 \pi \sigma) 3 / 2} \\
\times \frac{\exp \left[-\left(R-R_{0} v_{p} t\right)^{2} / 2 L\right]}{(2 \pi L) 3 / 2},
\end{aligned}
$$

where $L$ is defined by $L=1 / 4 \sigma$. We point out some interesting features of Eq. (2.16):

(a) It does not spread with time for fixed $p$, even though the underlying wave function does. However, contours of constant $f$, described by a generalized ellipse, do spread in the phase plane.

(b) The momentum and position distributions obey the uncertainty relation $\Delta p_{x} \Delta R_{x} \geq \frac{1}{2}$. Hence an equation like (2.7) is out of reach, except as a special limit involving $\hbar \rightarrow 0$.

(c) As $L$ tends to infinity $f_{0}$ becomes spread uniformly over space, while the momentum factor is sharp in momentum.

The Gaussian packet (2.16) is an especially good prototype incident-free packet. Since we shall use these results for large $L$, the normalizations appropriate to the continuum counterparts are listed (as $L \rightarrow \infty$ ):

$$
\begin{aligned}
& (L)^{3 / 4} C(\underline{p}) \rightarrow \delta\left(\underline{p}-\underline{p}_{0}\right), \\
& (L / 2 \pi)^{3 / 4} \psi_{p_{0}}(\underline{R}) \rightarrow \exp \left[\underline{i} \underline{p}_{0}\left(\underline{R}-\underline{R}_{0}\right)\right], \\
& (L / 2 \pi)^{3 / 2} f_{0}(\underline{p}, \underline{R}, t) \rightarrow \delta\left(\underline{p}-\underline{p}_{0}\right) /(2 \pi)^{3} .
\end{aligned}
$$

The indicated continuum normalization is $1 /(2 \pi)^{3}$ particles per unit volume. 
Other examples of analytically calculable phase-space distributions will be given in Sec. II.F.

Before taking up the question of computing $f(p, R, t)$, we explain how to express the result of a scattering experiment in the present formalism. We imagine (Fig. 1) a nearly uniform wave packet with momentum components $p \approx p_{0}$ incident on a localized potential $V$. The extent of the packet $L^{1 / 2}$ needs to be much larger than the size of the potential (called $a$ ) if we use Eq. (2.16) as our prototype packet. (Figure 1 has been drawn for $R_{0}$ equal to zero; for $L \rightarrow \infty$, the memory of $R_{0}$ disappears from the problem.) After the incident packet scatters off $V$, the outgoing signal is detected in the solid angle $d \Omega$ (Fig. 2). As time tends to infinity all particles pass by the detector. We rewrite Eq. (2.1) in differential form, giving

$$
\frac{d N_{\text {scatt }}}{d^{3} p}=\int d^{3} R f\left(\underline{p}, \underline{R},\left.t\right|_{t \rightarrow \infty} .\right.
$$

To calculate the incident flux and consequently the cross section, we note that the probability current is

$$
\begin{aligned}
\underline{j}(\underline{R}, t) & =\frac{1}{2 m i}\left(\psi^{*} \nabla \psi-\nabla \psi^{*} \psi\right) \\
& =\int d^{3} p \underline{v}_{p} f(\underline{p}, \underline{R}, t)
\end{aligned}
$$

the latter following an easy calculation. Therefore, the incident integrated flux along the beam direction $\hat{p}_{0}$ is

$$
\frac{d N_{\mathrm{inc}}}{d A}=\int_{\infty}^{\infty} d t \underline{j}_{0} \cdot \hat{p}_{0} f_{0}(p, R, t) \text {. }
$$

Using Eq. (2.14) for $f_{0}$ and noting that in the limit of sharp wave packets $\underline{p} \approx \underline{p}_{0}, q \approx 0$, we can derive

$$
\begin{aligned}
\frac{d N_{\text {inc }}}{d A}=\int d^{3} p \int & d^{3} q \delta\left(\underline{q} \cdot \hat{v}_{0}\right) C^{*}\left(\underline{p}-\frac{1}{2} q\right) \\
& \times C\left(\underline{p}+\frac{1}{2} q\right) /(2 \pi)^{2} .
\end{aligned}
$$

The differential cross section is then

$$
\frac{d \sigma}{d^{3} p}=\frac{\int d^{3} R f(p, R, \infty)}{d N_{\mathrm{inc}} / d A} .
$$

In this form one can take the continuum limit. Note that

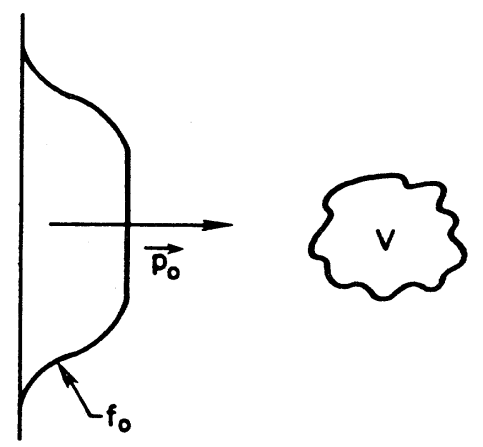

FIG. 1. A smooth free-particle Wigner distribution with momenta peaked near $\underline{p}_{0}$ approaching a localized potential $V$. since our $f$ includes the incident particles, the definition (2.22) does not agree with the traditional one in the forward direction $p_{0}$. This question will be clarified in Sec. II.E.

\section{B. Equation of motion: relation to the classical limit}

The equation of motion for $f$ follows directly from the Schrödinger equation,

$$
i \frac{\partial \psi(R, t)}{\partial t}=\left[\frac{-\nabla^{2}}{2 m}+V(\underline{R})\right] \psi(\underline{R}, t) .
$$

The result is more complicated formally, involving essentially the commutator of $H$ with the density matrix. But this form is directly related to the Poisson bracket form of classical mechanics, as shown below.

The kinetic term is most easily computed in momentum basis, giving

$$
\begin{aligned}
\int \frac{d^{3} q}{(2 \pi)^{3}} e^{i q \cdot R} & \left(\frac{\left(\underline{p}+\frac{1}{2} \underline{q}\right)^{2}}{2 m}-\frac{\left(\underline{p}-\frac{1}{2} \underline{q}\right)^{2}}{2 m}\right) C^{*}\left(\underline{p}-\frac{1}{2} \underline{q}, t\right) \\
& \times C\left(\underline{p}+\frac{1}{2} \underline{q}, t\right)=-\frac{i \underline{p}}{m} \nabla_{R} f(\underline{p}, R, t)
\end{aligned}
$$

The full equation of motion is then

$$
\begin{aligned}
\frac{D f}{D t} \equiv & \left(\frac{\partial}{\partial t}+\underline{v}_{p} \cdot \nabla\right) f(\underline{p}, \underline{R}, t) \\
= & \frac{1}{i \hbar} \int \frac{d^{3} r}{(2 \pi \hbar)^{3}} e^{-i \underline{p} \cdot \underline{r} / \hbar}\left[\underline{V}\left(\underline{R}+\frac{1}{2} \underline{r}\right)-V\left(\underline{R}-\frac{1}{2} r\right)\right] \\
& \times \psi^{*}\left(\underline{R}-\frac{1}{2} \underline{r}, t\right) \psi\left(\underline{R}+\frac{1}{2} \underline{r}, t\right) .
\end{aligned}
$$

We have temporarily reinstated the correct factors of $\hbar$ in order to facilitate our discussion of the classical limit. Equation (2.25) can be usefully rewritten as

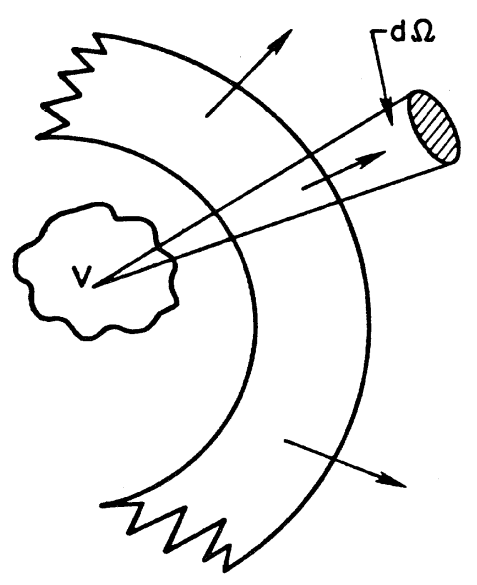

FIG. 2. The outgoing particle's density in phase space. This allows the evaluation of the cross section $d \sigma$ in the solid angle $d \Omega$. 


$$
\begin{aligned}
& \frac{D}{D t} f(p, R, t)=\frac{1}{i \hbar} \int \frac{d^{3} r d^{3} p^{\prime}}{(2 \pi \hbar)^{3}} e^{i\left(\underline{p^{\prime}}-\underline{p}\right) \cdot \underline{r}} \\
& \begin{aligned}
{\left[V\left(\underline{R}+\frac{1}{2} \underline{r}\right)-V\left(\underline{R}-\frac{1}{2} \underline{r}\right)\right] f\left(\underline{p}^{\prime}, \underline{R}, t\right) } \\
\quad \equiv \int d^{3} p^{\prime} K\left(\underline{p}^{\prime}-\underline{p}, \underline{R}\right) f\left(\underline{p^{\prime}}, \underline{R}, t\right),
\end{aligned}
\end{aligned}
$$

where the function $K$,

$$
\begin{aligned}
K\left(\underline{p^{\prime}}-\underline{p}, \underline{R}\right)=\frac{1}{i \hbar} \int \frac{d^{3} r}{(2 \pi \hbar)^{3}} e^{i\left(\underline{p}^{\prime}-\underline{p}\right) \underline{r} / \hbar}[ & V\left(\underline{R}+\frac{1}{2} \underline{r}\right) \\
& \left.-V\left(\underline{R}-\frac{1}{2} r\right)\right]
\end{aligned}
$$

occurs in many of our calculations. We shall also use the Fourier transform

$$
\begin{aligned}
i K\left(p^{\prime}-p, q\right)=V(q)[ & \delta\left(p^{\prime}-p+\frac{1}{2} q\right) \\
& \left.-\delta\left(p^{\prime}-p-\frac{1}{2} q\right)\right] .
\end{aligned}
$$

The difference of potentials occurring in Eq. (2.27) is important only for $r \lesssim a$, where $a$ is the range of the potential. Hence the significant momentum differences $\underline{p^{\prime}-p}$ occurring in $K$ are of order $\left|\underline{p}^{\prime}-\underline{p}\right| \lesssim \hbar / a$. Therefore, in the equation of motion (2.26), the potential has the effect of connecting $f$ 's with $p^{\prime}$ in the range $p^{\prime} \approx p \pm \hbar / a$. The finite contributions for $r= \pm 2 R \pm a$ oscillate if $R \gg a$ and do not contribute for short-range potentials.

In the classical limit, (2.26) becomes local in momentum space, too. In the limit $\hbar \rightarrow 0$, expanding

$$
V(R+1 / 2 r)-V(R-1 / 2 r) \cong \mathbf{r} \cdot \nabla V
$$

and writing $r=i \nabla_{p} / \hbar$ leads to the classical one-particle Liouville equation,

$$
\frac{\partial f}{\partial t}+\underline{v} \cdot \nabla f(\underline{p}, \underline{R}, t)-\nabla V(\underline{R}) \cdot \nabla_{p} f(\underline{p}, \underline{R}, t)=0 .
$$

Hence Eq. (2.26) may be regarded as a quantum Liouville equation. Information on classical scattering theory in the phase-space language can be found in Prigogine (1959) as well as in Miles and Dahler (1970) and $\mathrm{Eu}(1971,1973)$.

Equation (2.29) is equivalent to the constancy of the phase-space density $(d f / d t=0)$, provided the particles move on classical orbits specified by Newton's laws: $d \underline{r} / d t=\underline{p} / m, d \underline{p} / d t=-\nabla V . \quad$ The solution is then $f[\underline{p}(t), \underline{r}(t)]$.

If we keep all terms in the expansion of $V$, we easily derive

$$
\begin{aligned}
{\left[\frac{\partial}{\partial t}\right.} & +v \cdot \nabla] f(p, R, t) \\
& =\sum_{n \text { odd }} \frac{(i \hbar)^{n-1}}{2^{n-1} n !}\left(\nabla_{p} \cdot \nabla_{R}\right)^{n} V(R) f(p, R, t) \\
& =\frac{2}{\hbar} \sin \left[\frac{\hbar \nabla_{p} \cdot \nabla_{R}}{2}\right] V(R) f(p, R, t) .
\end{aligned}
$$

Here and subsequently it is understood that $\nabla_{R}$ acts only on $V$. The form (2.30) is most easily derived by writing

$$
V(\underline{R} \pm 1 / 2 \underline{r})=\exp ( \pm 1 / 2 \nabla) V
$$

in Eq. (2.27), which leads to

$$
K\left(p^{\prime}-p, R\right)=\frac{2}{\hbar} \sin \left(\frac{\hbar \nabla_{p} \cdot \nabla_{R}}{2}\right) V(R) \delta\left(p^{\prime}-p\right) .
$$

In either the quantum case [Eq. (2.26)] or the classical case [Eq. (2.29)] the motion is ballistic in the absence of a scattering potential. More precisely, the equation

$$
\frac{D}{D t} f=\left[\frac{\partial}{\partial t}+\underline{v}_{p} \cdot \nabla\right] f=0
$$

is solved by any function of $R-\underline{v}_{p} t$. The initial condition for the scattering problem is of this form.

The differential operator occurring in Eqs. (2.29) and (2.30) has the form

$\frac{2}{\hbar} \sin \left(\frac{\hbar \nabla_{p} \cdot \nabla_{R}}{2}\right)=D_{0}+\hbar^{2} D_{2}+\hbar^{4} D_{4}+\cdots$.

If we expand $f$ formally in a power series in $\hbar$,

$$
f=f_{0}+\hbar f_{1}+\hbar^{2} f_{2}+\cdots,
$$

we find the sequence of equations

$$
\begin{aligned}
& \left(\frac{D}{D t}-D_{0}\right) f_{0}=0, \\
& \left(\frac{D}{D t}-D_{0}\right) f_{1}=0, \\
& \left(\frac{D}{D t}-D_{0}\right) f_{2}=D_{2} f_{0}, \\
& \left(\frac{D}{D t}-D_{0}\right) f_{3}=D_{2} f_{1}, \\
& \left(\frac{D}{D t}-D_{0}\right) f_{4}=D_{2} f_{2}+D_{4} f_{0}, \\
& \left(\frac{D}{D t}-D_{0}\right) f_{5}=D_{2} f_{3}+D_{4} f_{1} .
\end{aligned}
$$

These equations are solved in sequence by simply integrating along the classical orbits, keeping in mind the initial distributions, whose $\hbar$ content will vary with the problem. For example, having found $f_{0}$, we calculate $f_{2}$ from the equation

$$
f_{2}=f_{2}^{(0)}+\int_{-\infty}^{t} d t^{\prime}\left(D_{2} f_{0}\right)\left[p\left(t^{\prime}\right), r\left(t^{\prime}\right)\right] .
$$

In this manner the entire quantum distribution is constructed in terms of the underlying classical orbits.

When the boundary condition is independent of $\hbar$, only even powers of $\hbar$ occur in the formal series (2.34). One of the first applications of the quantum phase-space distribution (Wigner, 1932) was the calculation of quantum corrections to the Boltzmann distribution. Although such results seem to make sense for thermally averaged quanti- 
ties, the formal expansion does not exist (Heller, 1976) in many important cases. The reason is that essential singularities spoil the expansion of Eqs. (2.34). A very simple example is the free Gaussian distribution, Eq. (2.16), in which the $\hbar$ dependence is of the form $h^{3} \exp \left(-A / \hbar^{2}\right)$. Hence every $\nabla_{p}$ brings in factors $\hbar^{-1}$ and $\hbar^{-2}$, canceling factors of $\hbar$ in the expansion (2.34). We refer the reader to the work of Heller (1976) for further information as well as for the description of a method to explicitly extract the singularities responsible for the failure of the naive approach outlined above. The essence of his method is to remove the essential singularities through a selective summation of the infinite series in $\hbar$. The problem is sufficiently important to deserve further study.

The phase-space distribution method has been used to illustrate various features of the (tricky) classical limit. The reader is referred to the following authors for further information and references: Heller (1977); Berry (1977); Berry and Balazs (1979); Berry, Balazs, Tabor, and Voros (1979). Particularly interesting is the semiclassical (WKB-derived) phase-space distribution derived and studied by Berry (1977). We expect that further development of these ideas for scattering theory will lead to important practical applications.

It is instructive to note that we can easily prove an "Ehrenfest theorem" using the Wigner distribution

$$
\frac{d\langle p\rangle}{d t}=\int d^{3} p d^{3} R \underline{p} \frac{\partial f(\underline{p}, \underline{R}, t)}{\partial t}=-\nabla V .
$$

A similar calculation for $\langle\underline{R}\rangle$ leads directly to

$$
\frac{d\langle\underline{R}\rangle}{d t}=\frac{\langle\underline{p}\rangle}{m} \text {. }
$$

For these simple moments, the classical procedure of computing expectation values coincides with the usual quantum-mechanical rules. For discussion of the generality of this, one should consult the references.

\section{Integral form of the scattering equations}

As is usual in scattering theory, it is useful to formally integrate the equation of motion to exhibit the incoming boundary condition explicitly. In anticipation of our later development, we pose the problem not only for $f(p, R, t)$, but for the corresponding space and time Fourier transforms.

Consider the equations

$$
\begin{aligned}
& \left(\frac{\partial}{\partial t}+\underline{v} \cdot \nabla\right] f(\underline{p}, \underline{R}, t)=S(\underline{p}, \underline{R}, t), \\
& {\left[i \frac{\partial}{\partial t}-\underline{q} \cdot \underline{v}\right] f(\underline{p}, \underline{q}, t)=i S(\underline{p}, \underline{q}, t),} \\
& (\omega-\underline{q} \cdot \underline{v}) f(\underline{p}, \underline{q}, \omega)=i S(\underline{p}, \underline{q}, \omega),
\end{aligned}
$$

$$
\begin{aligned}
\int_{-\infty}^{t} d t^{\prime} \int_{-\infty}^{t^{\prime}} d t^{\prime \prime} \int d^{3} p^{\prime} \int & d^{3} p^{\prime \prime} K\left[p^{\prime}-p, R-v_{p}\left(t-t^{\prime}\right)\right] K\left[p^{\prime \prime}-p^{\prime}, R-v_{p}\left(t-t^{\prime}\right)-v_{p}^{\prime}\left(t^{\prime}-t^{\prime \prime}\right)\right] \\
& \times f\left[p^{\prime \prime}, R-v_{p}\left(t-t^{\prime}\right)-v_{p}^{\prime}\left(t^{\prime}-t^{\prime \prime}\right), t^{\prime \prime}\right]
\end{aligned}
$$

$$
\begin{aligned}
& f(\underline{p}, \underline{q}, t) \equiv \int d^{3} R e^{-i \underline{q} \cdot \underline{R}} f(\underline{p}, \underline{R}, t), \\
& f(\underline{p}, \underline{q}, \omega) \equiv \int d^{3} R d t e^{i \omega t-i \underline{q} \cdot \underline{R}} f(\underline{p}, \underline{R}, t),
\end{aligned}
$$

with analogous expressions for the transforms of $S$.

The Green's functions corresponding to Eqs. (2.39) obey

$$
\begin{aligned}
& {\left[\frac{\partial}{\partial t}+\underline{v} \cdot \nabla\right] G(\underline{p}, \underline{R}, t)=\delta(\underline{R}) \delta(t),} \\
& {\left[i \frac{\partial}{\partial t}-\underline{q} \cdot \underline{v}\right] G(\underline{p}, \underline{q}, t)=i \delta(t),} \\
& (\omega-\underline{q} \cdot \underline{v}) G(\underline{p}, \underline{q}, \omega)=i .
\end{aligned}
$$

As usual, one has to define the pole suitable to the desired boundary condition (here retarded). The result is

$$
\begin{aligned}
& G_{\mathrm{ret}}(p, q, \omega)=\frac{i}{\omega+i \varepsilon-\underline{q} \cdot \underline{v}}, \\
& G_{\mathrm{ret}}(p, q, t)=\vartheta(t) e^{-i q \cdot v t}, \\
& G_{\mathrm{ret}}(\underline{p}, \underline{R}, t)=\vartheta(t) \delta(\underline{R}-\underline{v} t),
\end{aligned}
$$

when $\vartheta(t)$ is the usual step function: unity for $t>0$ and zero for $t<0$.

The integral forms of Eq. (2.36) are evidently

$$
\begin{aligned}
f(p, \underline{R}, t)= & f_{0}(\underline{p}, \underline{R}, t) \\
& +\int_{-\infty}^{t} d t^{\prime} S\left[\underline{p}, \underline{R}-\underline{v}_{p}\left(t-t^{\prime}\right), t^{\prime}\right], \\
f(\underline{p}, \underline{q}, t)= & f_{0}(\underline{p}, \underline{q}, t) \\
& +\int_{-\infty}^{t} d t^{\prime} e^{-i \underline{q} \cdot \underline{v}_{p}\left(t-t^{\prime}\right)} S\left(\underline{p}, \underline{q}, t^{\prime}\right), \\
f(\underline{p}, \underline{q}, \omega)= & f_{0}(\underline{p}, \underline{q}, \omega)+\frac{i S(\underline{p}, \underline{q}, \omega)}{\omega+i \varepsilon-\underline{q} \cdot \underline{v}} .
\end{aligned}
$$

Here $f_{0}$ solves the homogeneous equation suitable to the three descriptions: as $t \rightarrow-\infty, f \rightarrow f_{0}$. The retardation factors, which have an obvious physical meaning, find differing expression in the three forms of Eq. (2.39). Applying these results to the equation of motion (2.26) leads to the scattering integral equation

$$
\begin{aligned}
f(\underline{p}, \underline{R}, t)= & f_{0}(\underline{p}, \underline{R}, t) \\
& +\int_{-\infty}^{t} d t^{\prime} d^{3} p^{\prime} K\left[p^{\prime}-p, R-v_{p}\left(t-t^{\prime}\right)\right] \\
& \quad \times f\left[p^{\prime}, \underline{R}-\underline{v}_{p}\left(t-t^{\prime}\right), t^{\prime}\right] .
\end{aligned}
$$

Physically, it is clear that the values of $f$ at retarded points build up the whole function. The kinematicalkinetic content of Eq. (2.44) is exposed by iterating. To illustrate, the second-order term is 
Pictorially [Fig. 3(a)] the particle density begins at time $t^{\prime \prime}$, is deflected from $p^{\prime \prime}$ to $p^{\prime}$, propagating with velocity $v_{p}^{\prime}$ until $t^{\prime}$, when the potential changes the momentum to $p$. Then the density propagates between $t^{\prime}$ and $t$ with velocity $v_{p}$.

In $n$th order we have

$$
\int \prod_{i=i}\left(d t_{i} d^{3} p_{i}\right) K\left(p_{2}-p_{1}, R_{1}\right) \cdots K\left(p_{n}-p_{n-1}, R_{n}\right) f\left(p_{n}, R_{n}, t_{n}\right)
$$

where the variables are given by

$$
R_{1}=R-v_{p_{1}} \delta t_{1}, \delta t_{1}=t-t^{\prime}, \quad R_{2}=R_{1}-v_{p_{2}} \delta t_{2}, \delta t_{2}=t^{\prime}-t^{\prime \prime}, \quad R_{n}=R_{n-1}-v_{p n} \delta t_{n}, \delta t_{n+1}=t^{n}-t^{n+1} .
$$

The physical interpretation is again suggested by Fig. 3(b).

For many purposes the $(q, \omega)$ language is convenient. The scattering integral equation is

$$
f(p, q, \omega)=f_{0}(p, q, \omega)+\frac{1}{\omega+\underline{i} \varepsilon-\underline{q} \cdot \underline{v}_{p}} \int \frac{d^{3} q^{\prime}}{(2 \pi)^{3}} V\left(q^{\prime}\right)\left[f\left(p-\frac{1}{2} q^{\prime}, q-q^{\prime}, \omega\right)-f\left(p+\frac{1}{2} q^{\prime}, q-q^{\prime}, \omega\right)\right],
$$

where the incoming distribution function is

$$
f_{0}(p, q, \omega)=2 \pi \delta\left(\omega-\underline{q} \cdot \underline{v}_{p}\right) C^{*}\left(p-\frac{1}{2} q\right) C\left(p+\frac{1}{2} q\right) .
$$

It is easy to show that the differential cross section is the residue of a pole in $f(p, q, \omega)$ at $\omega=0$ for $\underline{q}=0$. For $\underline{p} \neq \underline{p_{0}}$ we have

$$
\left.\int d^{3} R f(\underline{p}, \underline{R}, t)\right|_{t=\infty}=f(\underline{p}, \underline{q}=0, t=\infty)=\int d t \frac{\partial f}{\partial t}(\underline{p}, \underline{q}=0, t)=-i[\omega f(\underline{p}, \underline{q}=0, \omega)]_{\omega=0} .
$$

It is instructive, though tedious, to solve Eq. (2.44) by iteration,

$$
f=f_{0}+f_{1}+f_{2}+\cdots,
$$

where

$$
f_{n+1}(\underline{p}, \underline{q}, \omega)=\frac{1}{\omega+i \varepsilon-\underline{q} \cdot \underline{v}_{p}} \int \frac{d^{3} q^{\prime}}{(2 \pi)^{3}} V\left(q^{\prime}\right)\left[f_{n}\left(p-\frac{1}{2} q^{\prime}, q-q^{\prime}, \omega\right)-f_{n}\left(p+\frac{1}{2} q^{\prime}, q-q^{\prime}, \omega\right)\right] .
$$

Note that since $f_{0}$ exhausts the normalization we have

$$
\int f_{n}(p, R, t) d^{3} p d^{3} R=0, \quad n \geq 1 .
$$

$f_{1}$ does not contribute to the cross section as anticipated (it is linear in $V$ ). Working through the details, one finds the flux factor (2.21) explicitly, giving in second order

$$
\frac{v d \sigma}{d^{3} p}=(2 \pi)^{-2}\left|V\left(\underline{p}-\underline{p}_{0}\right)\right|^{2} \delta\left(E-E_{0}\right) .
$$

Rather than derive this explicitly, we shall show how our formalism leads to the general formula wherein the Born cross section (2.52) is replaced by the identical formula with $V$ replaced by the transition matrix. Our calculation is somewhat similar to that of Gottfried (1966), who uses the density matrix.

\section{Exact formula for the scattering cross section}

In order to derive the generalization $(2.48)$, we begin by expressing the Fourier transform of (2.11) in Dirac's bracket notation,

$$
\begin{aligned}
f(\underline{p}, \underline{q}, t) & =C\left(\underline{p}+\frac{1}{2} \underline{q}, t\right) C^{*}\left(\underline{p}-\frac{1}{2} \underline{q}, t\right) \\
& =\left\langle\underline{p}+\frac{1}{2} \underline{q} \mid \psi(t)\right\rangle\left\langle\psi(t) \mid p-\frac{1}{2} \underline{q}\right\rangle .
\end{aligned}
$$

The wave function $\psi$ obeys the usual equation

$$
\psi(t)=\varphi(t)-i \int_{-\infty}^{t} d t^{\prime} e^{-i H_{0}\left(t-t^{\prime}\right)} V \psi\left(t^{\prime}\right),
$$

where $\psi \rightarrow \varphi$ as $t \rightarrow-\infty$ and the exponential oscillations are to be tamed by the adiabatic hypothesis. We can ex-

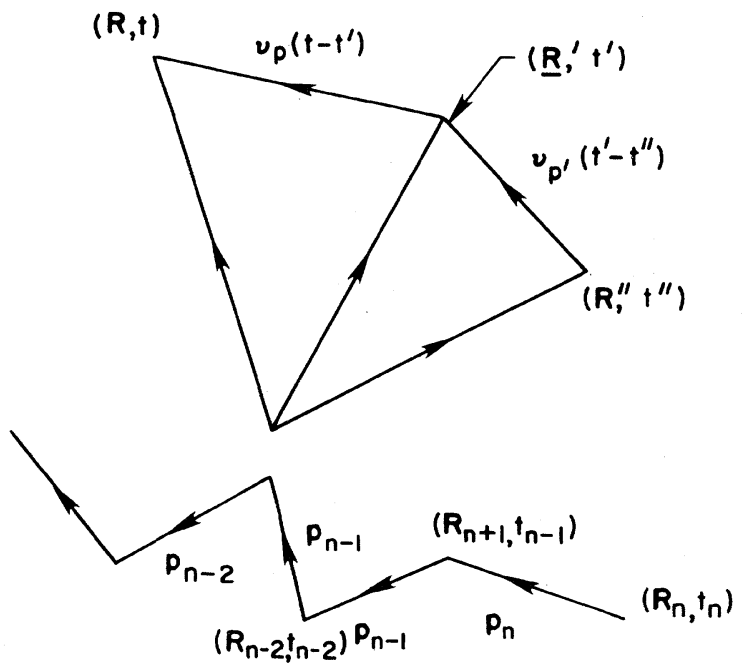

FIG. 3. Iterative contributions to the scattering integral equation. (a) The structure of the second-order iterate [Eq. (2.45)]. The retardation terms propagate the phase-space densities freely between interactions with the potential. (b) The structure of the $n$ th-order iterate. 
pand $\psi$ and $\varphi$ in terms of the stationary wave functions $\exp (i \underline{k} \cdot \underline{x})$ and $\psi_{k}^{+}(\underline{x})$, respectively, using the same expansion coefficient $\chi(p)$ :

$$
\begin{aligned}
& \varphi(\underline{x}, t)=\int \frac{d^{3} p}{(2 \pi)^{3 / 2}} \chi(\underline{p}) e^{i \underline{p} \cdot \underline{x}-i E} p^{t}, \\
& \psi(\underline{x}, t)=\int \frac{d^{3} p}{(2 \pi)^{3 / 2}} \chi(\underline{p}) \psi_{p}^{+}(\underline{x}) e^{-i E} p^{t},
\end{aligned}
$$

where $E_{p}=p^{2} / 2 m$. For a sharp $\chi(p)=(2 \pi)^{3 / 2} \delta(p-k)$, we find the usual result

$$
\psi_{k}^{+}(\underline{x})=e^{i \underline{k} \cdot \underline{x}}+\frac{1}{E_{k}+i \varepsilon-H_{0}} V \psi_{k}^{+}(x) .
$$

The usual transition matrix for scattering from momentum state $k$ to $k^{\prime}$ is

$$
T_{k^{\prime}, k} \equiv\left\langle k^{\prime}|V| \psi_{k}^{+}\right\rangle \text {. }
$$

In our calculations it will be useful to use the related amplitude

$$
\begin{aligned}
T_{p}(t) & =\langle p|V| \psi(t)\rangle \\
& =\int \frac{d^{3} k}{(2 \pi)^{3 / 2}} \chi_{p_{0}}(k) e^{-i E_{k} t}\left\langle p|V| \psi_{k}^{+}\right\rangle .
\end{aligned}
$$

Here we have labeled $\chi$ with a momentum $p_{0}$, about which we shall eventually make $\chi_{p_{0}}(k)$ peak. The equation of motion is

$$
\left[i \frac{\partial}{\partial t}-\underline{v}_{p} \cdot \underline{q}\right] f(\underline{p}, \underline{q}, t)=\left\langle p+\frac{1}{2} q|V| \psi(t)\right\rangle\left\langle\psi(t) \mid p-\frac{1}{2} q\right\rangle-\left\langle p+\frac{1}{2} q \mid \psi(t)\right\rangle\left\langle\psi(t)|V| p-\frac{1}{2} q\right\rangle .
$$

As it stands, Eq. (2.59) exhibits only one explicit $V$ on the right-hand side. But the desired structure, containing two $V$ 's and two $\psi$ 's, results immediately on substituting the integral equation (2.50), converting the right-hand side of Eq. (2.59) to:

$$
\begin{aligned}
& \left\langle p+\frac{1}{2} q|V| \psi\right\rangle\left\langle\varphi \mid p-\frac{1}{2} q\right\rangle-\left\langle p+\frac{1}{2} q \mid \varphi\right\rangle\left\langle\psi|V| p-\frac{1}{2} q\right\rangle \\
& \quad+i\left\langle p+\frac{1}{2} q|V| \psi(t)\right\rangle \int_{-\infty}^{t} d t^{\prime} \exp \left[i E_{p-q / 2}\left(t-t^{\prime}\right)\right]\left\langle\psi\left(t^{\prime}|V| p-\frac{1}{2} q\right\rangle\right. \\
& \quad+i \int_{-\infty}^{t} d t^{\prime} \exp \left[-i E_{p+q / 2}\left(t-t^{\prime}\right)\right]\left\langle p+\frac{1}{2} q|V| \psi\left(t^{\prime}\right)\right\rangle\left\langle\psi(t)|V| p-\frac{1}{2} q\right\rangle .
\end{aligned}
$$

The first two terms are nonzero only in the forward direction $p_{0}$ for $q=0$ and will be dropped here. The time integrals in the third and fourth terms are evaluated using Eq. (2.55). Further performing the time Fourier transform gives

$$
\begin{aligned}
2 \pi \int & \frac{d^{3} k d^{3} k^{\prime}}{(2 \pi)^{3}} \chi_{p_{0}}(k) \chi_{p_{0}}^{*}\left(k^{\prime}\right) \delta\left(\omega+E_{k^{\prime}}-E_{k}\right) \\
& \times\left(\frac{-1}{E_{k}-E_{p+q / 2}-i \varepsilon}+\frac{1}{E_{k^{\prime}}-E_{p-q / 2}+i \varepsilon}\right) \\
& \times\left\langle p+\frac{1}{2} q|V| \psi_{k}^{+}\right\rangle\left\langle\psi_{k^{\prime}}^{+}|V| p-\frac{1}{2} q\right\rangle
\end{aligned}
$$

Making the substitutions $k=P+Q / 2, k^{\prime}=P-Q / 2$, we can express Eq. (2.59) in the form

$$
\begin{aligned}
& \int \frac{d^{3} P d^{3} Q}{(2 \pi)^{3}} \chi^{*}\left(\underline{P}-\frac{1}{2} \underline{Q}\right) \chi\left(\underline{P}+\frac{1}{2} \underline{Q}\right) \delta(\omega+\underline{P} \cdot \underline{Q} / m) \\
& \quad \times\left(\frac{1}{E_{P-Q / 2}-E_{p-q / 2}+i \varepsilon}-\frac{1}{E_{\underline{P}+Q / 2}-E_{p-q / 2}-i \varepsilon}\right) \\
& \quad \times\left\langle p+\frac{1}{2} q|V| \psi_{P+Q / 2}^{+}\right\rangle\left\langle\psi_{P}^{+}-Q / 2|V| p-\frac{1}{2} q\right\rangle .
\end{aligned}
$$

We are interested in the limit of this expression for $\underline{q}=\omega=0$ and for the wave-packet coefficients $\chi_{p_{0}}$ sharply peaked around $p_{0}$. This allows us to set $Q=0, p=\underline{p}_{0}$ in slowly varying functions. Hence for $\underline{p} \neq \underline{p}_{0}$ we find

$$
\begin{aligned}
-i[\omega f(\underline{p}, \underline{0}, \omega)]_{\omega=0}= & 2 \pi \delta\left(E_{p_{0}}-E_{p}\right)\left|\left\langle p|V| \psi_{p_{0}}^{+}\right\rangle\right|^{2} \\
& \times \int \frac{d^{3} P d^{3} Q}{(2 \pi)^{3}} \chi *\left(P-\frac{1}{2} Q\right) \\
& \times \chi\left(P+\frac{1}{2} Q\right) \delta(\mathbf{P} \cdot \mathbf{Q} / m) .
\end{aligned}
$$

Recognizing the flux factor from Eq. (2.21) and using (2.49) leads directly to

$$
\frac{v d \sigma}{d^{3} p}=(2 \pi)^{-2} \delta\left(E-E_{0}\right)\left|\left\langle p|V| \psi_{p_{0}}^{+}\right\rangle\right|^{2},
$$

where $v=p_{0} / m$ is the incident velocity.

We now clarify the special features of the wave interference behavior in the forward direction and in so doing show how the optical theorem arises in the present formalism. Our previous definition of the cross section (2.22) was unorthodox in counting all final particles, including the incident beam. This approach is too tied to the classical approach and obscures the important phenomenon of diffraction scattering.

To clarify this question, we note that corresponding to the decomposition

$$
\psi=\varphi+\psi_{\mathrm{sc}},
$$

to which (2.54) tends as $t \rightarrow \infty$, we have for the Wigner distribution 


$$
f=f_{0}+f_{\mathrm{int}}+f_{\mathrm{sc}},
$$

where $f_{0}, f_{\text {int }}$, and $f_{\text {sc }}$ are, respectively, the incident, interference, and scattered components. In the equation of motion $f_{0}$ drops out. In Eq. (2.60) the first two terms dropped are exactly the interference terms which arise from the overlap of the incident and scattered wave. Therefore, the necessary correction to Eq. (2.22) is the subtraction not only of the $f_{0}$ contribution (exactly as in classical mechanics), but also of the interference term. From Eq. (2.60) we see that this interference term is just the imaginary part of the $T$ matrix, as one should expect, in the limit $q \rightarrow 0$.

The continuum limit of Eq. (2.60) is easy to take. In that limit the various states become energy-momentum eigenstates and the left-hand side vanishes as $\underline{q} \rightarrow 0$. The vanishing of the right-hand side then produces the optical theorem as a constraint. From Eq. (2.56) we see that a suitable normalization for continuum states is $(2 \pi)^{3} \delta\left(\underline{p}^{\prime}-\underline{p}\right)$. Performing the trivial time integrations gives

$$
\begin{aligned}
(2 \pi)^{3} \delta\left(\underline{p}-\underline{p}_{0}\right)\left[\left\langle p|T| p_{0}\right\rangle-\left\langle p|T| p_{0}\right\rangle^{*}\right] & \\
& +2 \pi i \delta\left(E-E_{0}\right)\left|T_{p p_{0}}\right|^{2}=0 .
\end{aligned}
$$

Integration over $d^{3} p$ gives the usual form,

$$
\operatorname{Im} T_{p_{0} p_{0}}=-\pi \int \frac{d^{3} p}{(2 \pi)^{3}} \delta\left(E-E_{0}\right)\left|T_{p, p_{0}}\right|^{2} .
$$

\section{E. The eikonal approximation}

High-energy, small-angle potential scattering is well described by the eikonal approximation (Glauber, 1959). This result can be easily derived in the present formalism by one simple approximation, i.e., replacing the velocity in the drift term $v \cdot \nabla$ by the incoming velocity $v_{0}=p_{0} / m$.

We write the equation of motion in the form (2.26)

$$
i\left(\frac{\partial}{\partial t}+v_{0} \cdot \nabla\right) f(p, R, t)=\int \frac{d^{3} r d^{3} p^{\prime}}{(2 \pi)^{3}} e^{i\left(\underline{p^{\prime}}-\underline{p}\right) \cdot r}\left[V\left(\underline{R}+\frac{1}{2} r\right)-V\left(\underline{R}-\frac{1}{2} r\right)\right] f\left(p^{\prime}, R, t\right) .
$$

Noting the convolution structure in momentum, we can rewrite this in terms of

as

$$
f(y, R, t)=\int \frac{d^{3} p}{(2 \pi)^{3}} e^{i \underline{p} \cdot \underline{y}} f(\underline{p}, \underline{R}, t)
$$

$$
i\left(\frac{\partial}{\partial t}+v_{0} \cdot \nabla\right) f(y, R, t)=\left[V\left(R+\frac{1}{2} y\right)-V\left(R-\frac{1}{2} y\right)\right] f(y, R, t) .
$$

[Note that the exact equation corresponds to replacing $v_{0}$ by $-i \nabla_{y}$ in Eq. (2.71).]

Equation (2.71) is solved by integration,

$$
f(y, R, t)=f_{0}(y, R, t) \exp \left[-i \int_{-\infty}^{t} d t^{\prime}\left\{V\left[R+\frac{1}{2} y-v_{0}\left(t-t^{\prime}\right)\right]-V\left[R-\frac{1}{2} y-v_{0}\left(t-t^{\prime}\right)\right]\right\}\right],
$$

where $f_{0}$ is as usual the incoming density function, transformed as in Eq. (2.66). Therefore, the desired phase-space density is

$$
f(p, R, t)=\int d^{3} y e^{-i p \cdot y} f_{0}(y, R, t) \exp \left[-i \int_{-\infty}^{t} d t^{\prime}\left\{V\left[R+\frac{1}{2} y-v_{0}\left(t-t^{\prime}\right)\right]-V\left[R-\frac{1}{2} y-v_{0}\left(t-t^{\prime}\right)\right]\right\}\right] .
$$

Defining variables parallel and perpendicular to $v_{0}$ in the obvious way and writing $z=R_{\|}-v_{0}\left(t-t^{\prime}\right)$ allows us to rewrite Eq. (2.73) as

$$
f(p, R, t)=\int d^{3} y e^{-i p \cdot y} f_{0}(y, R, t) \exp \left\{\frac{-i}{v_{0}} \int_{-\infty}^{R_{\|}} d z\left[V\left[z+\frac{y_{\|}}{2}, R_{\perp}+\frac{y_{\perp}}{2}\right)-V\left(z-\frac{y_{\|}}{2}, R_{\perp}-\frac{y_{\perp}}{2}\right)\right]\right\} .
$$

Now all the time dependence has been placed in an especially simple, controllable function, namely, the free motion described by $f_{0}$.

We now use the Gaussian $f_{0}$ of Eq. (2.16) to simplify (2.74). We find, setting $R_{0}=0$,

$$
f(y, R, t)=\frac{1}{(2 \pi)^{3}} e^{i p_{0} \cdot y} e^{-\sigma y^{2} / 2} \frac{e^{-\left(R-v_{0} t\right)^{2} / 2 L}}{(2 \pi L)^{3 / 2}},
$$

where eventually we shall want to take the limits $\sigma \rightarrow 0$, $L \rightarrow \infty$. To obtain the cross section, we need to integrate Eq. (2.74) over $R$ and study the behavior as to $t \rightarrow \infty$. To accommodate the structure of the potential term, it is use- ful to change variables as follows:

$$
\begin{aligned}
& y_{1}=R+\frac{1}{2} y, \\
& y_{2}=R-\frac{1}{2} y .
\end{aligned}
$$

Further writing

$$
\begin{aligned}
& y_{1_{||}}=z_{1}, y_{\perp}=\underline{b}_{1}, \\
& y_{2_{||}}=z_{2}, y_{2_{1}}=\underline{b}_{2}
\end{aligned}
$$

(the $b_{i}$ will be impact parameters), we obtain after a little arithmetic 


$$
\begin{aligned}
& \int d^{3} R f(p, R, t)=\int d^{2} b_{1} d^{2} b_{2} \exp \left[-i\left(p-p_{0}\right)_{\perp} \cdot\left(b_{1}-b_{2}\right)\right] \exp \left[-\left(\frac{b_{1}+b_{2}}{2}\right)^{2} / 2 L\right] \\
& \times \exp \left[-\sigma\left(b_{1}-b_{2}\right)^{2} / 2\right] \int d z_{1} d z_{2} \exp \left[-i\left(p-p_{0}\right)_{\|}\left(z_{1}-z_{2}\right)\right] \exp \left[-\left(\frac{z_{1}+z_{2}}{2}-v_{0} t\right]^{2} / 2 L\right] \\
& \times \exp \left[-\sigma\left(z_{1}-z_{2}\right)^{2} / 2\right] \Phi\left(z_{1}, b_{1}\right) \Phi^{*}\left(z_{2}, b_{2}\right) /(2 \pi)^{3}(2 \pi L)^{3 / 2},
\end{aligned}
$$

where the function $\Phi$ is defined by

$$
\Phi(z, b) \equiv \exp \left[-\frac{i}{v_{0}} \int_{-\infty}^{z_{1}} d z V(z, b)\right] .
$$

The complex expression (2.79) simplifies greatly in the limit $t \rightarrow \infty$, since then the only contributions come from $z_{1}+z_{2} \rightarrow \infty$, too. Actually, $z_{1}$ and $z_{2}$ tend to $+\infty$ together for finite $\sigma$ because of the factor $\exp \left[-\sigma\left(z_{1}-z_{2}\right)^{2} / 2\right]$. For finite-range potentials $\Phi(z, b)=\Phi(\infty, b)$ as soon as $z$ exceeds the potential range. Hence the $z_{1}, z_{2}$ dependence of the potential functions $\Phi$ disappears as $t \rightarrow \infty$. Going over to coordinates $z=z_{1}-z_{2}, Z=\left(z_{1}+z_{2}\right) / 2$, we can do the two integrations explicitly,

$$
\begin{aligned}
\int d Z \frac{e^{-\left(Z-v_{0} t\right)^{2} / 2 L}}{(2 \pi L)^{1 / 2}} \int d z e^{-(\sigma / 2) z^{2}} e^{-\left(p-p_{0}\right) \|^{z}} \\
\approx 2 \pi \delta\left(p_{\|}-p_{0}\right) \approx 2 \pi v_{0} \delta\left(E-E_{0}\right),
\end{aligned}
$$

reinterpreting the $\sigma \rightarrow 0$ integral as an energy-conserving delta function.

Letting $\sigma \rightarrow 0, L \rightarrow \infty$ inside the $\underline{b}$ integrations, we find

$$
\begin{aligned}
\int d^{3} R f(p, R, \infty)= & \frac{1}{2 \pi L} \frac{1}{(2 \pi)^{3}} 2 \pi v_{0} \delta\left(E-E_{0}\right) \\
& \times\left|\int d^{2} b e^{-i \underline{\Delta} \cdot \underline{b}} \Phi(\infty, \underline{b})\right|^{2},
\end{aligned}
$$

where $\Delta \equiv p-p_{0}$ is the momentum transfer.

The coefficient $1 /(2 \pi L)$ is nothing but the flux factor (2.20) evaluated for the Gaussian as $L \rightarrow \infty$ :

$$
\left\lfloor\frac{d N}{d A}\right)=\exp \left(-R_{\perp}^{2} / 2 L\right) / 2 \pi L \rightarrow \frac{1}{2 \pi L} .
$$

Finally, writing

$$
d^{3} p=p^{2} \frac{d p}{d E} d E d \Omega
$$

and integrating over $E$ gives

$$
\left.\frac{d \sigma}{d \Omega}=\left|\frac{p}{2 \pi} \int d^{2} b e^{-i \Delta \cdot b} \exp \right|-\frac{i}{v_{0}} \int_{-\infty}^{\infty} V(Z, b) d Z\right)\left.\right|^{2} .
$$

This formula differs from the usual one by the absence of a -1 accompanying the exponential. The -1 comes from subtracting the incident wave to define a scattered amplitude. Our formalism, which involves the full $f$, includes everything, and therefore the definition (2.22) does not coincide with the traditional definition of the cross section in exactly the forward direction. That is, of course, where the discrepancy lies, in that the -1 term produces a $\delta(\Delta)$ on performing the $b$ integration. Equation (2.80) involves the full $f$, while squaring $\Phi-1$ leads to an immediate identification with the terms in Eq. (2.66).

\section{F. The bound-state problem}

In the preceding sections we have formulated the quantum scattering problem in terms of the time-dependent solutions to the Liouville equation

$$
L f=i \frac{\partial f}{\partial t},
$$

where the quantum Liouville operator $L$ is defined by

$$
\begin{aligned}
L(p, R) f(p, R, t)= & -i v_{p} \cdot \nabla_{R} f(p, R, t) \\
& +i \int d^{3} p^{\prime} K\left(p^{\prime}-p, R\right) f\left(p^{\prime}, R, t\right) .
\end{aligned}
$$

For energy eigenstates, $\psi_{n} \sim e^{-i E_{n} t} f$ is time independent, so that $f_{n n}$ constructed from such wave functions must satisfy

$$
L f_{n n}=0 \text {. }
$$

It might be imagined that Eq. (2.84) is a combined eigenfunction-eigenvalue problem for $E_{n}$ and the associated phase-space distributions. It turns out that, in contrast to the scattering problem (for which the boundary conditions are different), the Liouville equation does not determine the Wigner function in this case.

A first indication of this comes from a closer inspection of the equation of motion (2.45) in the case when $f$ has no explicit time dependence. For bound states there is, in addition, no incident wave packet. Shifting variables to $\tau=t^{\prime}-t$ gives the bound-state equation

$$
\begin{aligned}
f_{B}(p, R)=\int_{-\infty}^{0} d \tau \int & d^{3} p^{\prime} K\left(\underline{p}^{\prime}-\underline{p}, \underline{R}+\underline{v}_{p} \tau\right) \\
& \times f\left(\underline{p^{\prime}}, \underline{R}-\underline{v}_{p} \tau\right) .
\end{aligned}
$$

In Fourier transform language this becomes

$$
\begin{aligned}
& \underline{q} \cdot \underline{v}_{p} f_{B}(p, q)=\int \frac{d^{3} q^{\prime}}{(2 \pi)^{3}} V\left(q^{\prime}\right)[ f_{B}\left(p+\frac{1}{2} q^{\prime}, q-q^{\prime}\right) \\
&\left.-f_{B}\left(p-\frac{1}{2} q^{\prime}, q-q^{\prime}\right)\right]
\end{aligned}
$$


in agreement with Eq. (2.47) adapted to bound states. To illuminate this equation further, we rederive Eq. (2.85) from the bound-state Schrödinger equation in momentum space:

$$
\left[E-\frac{q^{2}}{2 m}\right] \chi(q)=\int \frac{d q^{\prime}}{(2 \pi)^{3}} V\left(q^{\prime}\right) \chi\left(q-q^{\prime}\right),
$$

where the $\chi$ 's are defined (and called $C$ ) in Eq. (2.2). From Eq. (2.11), we find for $f(p, q)$

$$
f(p, q)=\chi *\left(p-\frac{1}{2} q\right) \chi\left(p+\frac{1}{2} q\right) \text {. }
$$

We now construct $f(p, q)$ in two ways using Eq. (2.86):

$$
\begin{aligned}
\left(E-E_{p-q / 2}\right) \chi *\left(p-\frac{1}{2} q\right) \chi\left(p+\frac{1}{2} q\right) & \\
= & \int \frac{d q^{\prime}}{(2 \pi)^{3}} V\left(q^{\prime}\right) \chi^{*}\left(p-\frac{1}{2} q+q^{\prime}\right) \chi\left(p+\frac{1}{2} q\right), \\
\left(E-E_{p+q / 2}\right) \chi & *\left(p-\frac{1}{2} q\right) \chi\left(p+\frac{1}{2} q\right) \\
& =\int \frac{d q^{\prime}}{(2 \pi)^{3}} V\left(q^{\prime}\right) \chi^{*}\left(p-\frac{1}{2} q\right) \chi\left(p+\frac{1}{2} q-q^{\prime}\right) .
\end{aligned}
$$

Identifying the integrands as $f\left(p+\frac{1}{2} q^{\prime}, q-q^{\prime}\right)$ and $f\left(p-\frac{1}{2} q^{\prime}, q-q^{\prime}\right)$ and subtracting gives Eq. (2.85), since

$$
E_{p+q / 2}-E_{p-q / 2}=p \cdot q / m \text {. }
$$

The cancellation of the energy eigenvalue in going from Eq. (2.88) to Eq. (2.85) leads to the suspicion that the latter may not, in general, determine the energies or eigenfunctions of the bound states. This is in contrast to Eq. (2.86), which poses a standard eigenvalue-eigenfunction problem.

In order to clarify this situation, we note that the basic equation of motion (2.81) is a particular realization of the density-matrix equation of motion

$$
i \frac{\partial \rho}{\partial t}=[H, \rho]
$$

As a consequence, the solution to the more general eigenvalue problem

$$
L f_{\lambda}=\lambda f_{\lambda}
$$

( $\lambda$ real but not necessarily positive) is easily surmised by inspection of Eq. (2.91). Writing $\rho_{m n}=|m\rangle\langle n|$, where $|n\rangle,|m\rangle$ represent energy eigenstates, we see that

$$
i \frac{\partial \rho_{m n}}{\partial t}=\left(E_{m}-E_{n}\right) \rho_{m n} \text {. }
$$

Recalling the relation between the density matrix and the Wigner distribution [Eq. (2.10)], we see that the eigenfunctions of $L$ corresponding to Eq. (2.91) are the offdiagonal generalization of $f$ :

$$
\begin{aligned}
& f_{m n}(p, R, t) \equiv \int \frac{d^{3} r}{(2 \pi)^{3}} e^{-i \underline{p} \cdot r} \psi_{n}^{*}\left(R-\frac{1}{2} r, t\right) \\
& \times \psi_{m}\left(R+\frac{1}{2} r, t\right), \\
& L f_{m n}=\left(E_{m}-E_{n}\right) f_{m n}, \\
& f_{m n}(t)=e^{-i\left(E_{m}-E_{n}\right) t} f_{m n}(0),
\end{aligned}
$$

where the phase-space integral of $f_{m n}$ vanishes. We now see that the energy eigenvalues are to be constructed from the eigenvalue spectrum of $L$.

Since the $\rho_{m n}$ form a complete set of matrices by which any operator can be represented, so do the $f_{m n}$ of Eq. (2.92) form a complete set. In particular, a general $f$ can be represented as

$$
f(p, R, t)=\sum_{m n} C_{m n} f_{m n}(p, R, t) .
$$

It is useful to state here the completeness and orthogonality conditions obeyed by the $f_{m n}$. (For this purpose, we remove the time dependence of the energy eigenstates.) Straightforward calculations using the completeness and orthogonality of the wave functions lead to

$$
\begin{aligned}
& \int d^{3} p d^{3} R f_{m n}(p, R) f_{\mathrm{st}}^{*}(p, R)=\delta_{n t} \delta_{s m} /(2 \pi)^{3} \\
& \sum_{m n} f_{m n}(p, R) f_{m n}^{*}\left(p^{\prime}, R^{\prime}\right)=\delta\left(R-R^{\prime}\right) \delta\left(p-p^{\prime}\right) /(2 \pi)^{3} .
\end{aligned}
$$

In the particularly interesting case when the system is subjected to an external potential $V_{\mathrm{ex}}(\underline{R}, t)$, the $C_{m n}$ 's become time dependent and induce transitions among the various "stationary" solutions $f_{m n}$ (cf. Sec. II.H).

The construction (2.92) provides the desired solution to Eq. (2.90). However, an attempt to solve (2.90) does not lead uniquely to Eq. (2.92). A symptom of this difficulty is easily seen from the form of the density-matrix analog to Eq. (2.90):

$$
\left[H, \rho_{\lambda}\right]=\lambda \rho_{\lambda}
$$

Clearly any solution to this equation can be multiplied by an arbitrary function of the constants of the motion without changing the eigenvalues.

The problem evidently is that the single equation (2.90) is not completely equivalent to the Schrödinger equation. The proper resolution of this situation has been clearly laid out in a recent paper by Dahl (1981). In addition to the commutator structure $L f \leftrightarrow[H, \rho]$, we need the anticommutator analog $\widetilde{L} \widetilde{f} \leftrightarrow \frac{1}{2}[H, \rho]_{+}$, with the associated eigenvalue problem

$$
\tilde{L} \widetilde{f}_{\tilde{\lambda}}=\tilde{\lambda} \widetilde{f}_{\tilde{\lambda}}
$$

Clearly we can reconstruct the usual Schrödinger eigenvalue problem from $L$ and $\widetilde{L}$. The $(p, R)$ form for $\widetilde{L}$ is easily obtained by suitably changing signs in Eqs. (2.24) and (2.25). Noting that $f_{m n}$ also solves Eq. (2.96),

$$
\widetilde{L} f_{m n}=\frac{1}{2}\left(E_{m}+E_{n}\right) f_{m n},
$$

we find for the kinetic energy term

$$
\frac{1}{2}[T, \rho]_{+} \rightarrow \frac{p^{2}}{2 m} f_{m n}-\frac{1}{8 m} \nabla_{R}^{2} f_{m n} .
$$

Combining this with the potential energy term, we find 


$$
\begin{aligned}
\frac{1}{2}\left(E_{m}+E_{n}\right) f_{m n}= & {\left[\frac{p^{2}}{2 m}-\frac{1}{8 m} \nabla_{R}^{2}\right] f_{m n} } \\
& +\int d^{3} p^{\prime} \widetilde{K}\left(p^{\prime}-p, R\right) f_{m n}\left(p^{\prime}, R\right),
\end{aligned}
$$

where the kernel $\widetilde{K}$ is given by

$$
\begin{aligned}
\widetilde{K}\left(p^{\prime}-p, R\right)=\frac{1}{2} \int & \frac{d^{3} r}{(2 \pi)^{3}} e^{i\left(p^{\prime}-p\right) \cdot r} \\
& \times\left[V\left(R+\frac{1}{2} r\right)+V\left(R-\frac{1}{2} r\right)\right] .
\end{aligned}
$$

Note that the time dependence of $f_{m n}$ cancels out in Eq. (2.99).

Equations (2.90) and (2.99) provide the required constraints to solve the eigenvalue-eigenfunction (matrix) problem. Note that $f_{m n}$ will have to vanish for $r \rightarrow \infty$, $p \rightarrow \infty$ if $m$ and $n$ are both bound states. In the continuum, of course, the eigenvalue is arbitrary and Eq. (2.91) suffices to determine the solution to the scattering problem.

If we expand in $\underline{r}$, we find

$\widetilde{K}=V(R) \delta\left(p^{\prime}-p\right)-\frac{1}{8} V_{i j}^{\prime \prime}(R) \frac{\partial}{\partial p_{i}} \frac{\partial}{\partial p_{j}} \delta\left(p^{\prime}-p\right)+\cdots$

Note that the leading term is just

$H_{\text {class }}(p, R) f_{m n}=\left(\frac{p^{2}}{2 m}+V(R)\right) f_{m n}=\frac{1}{2}\left(E_{m}+E_{n}\right) f_{m n}$,

the classical equation of motion, whose energy is the average of the two quantum energies.

In order to demonstrate this and other points, we examine the one-dimensional harmonic oscillator. In coordinate space, the stationary Liouville equation is

$$
\frac{p}{m} \frac{\partial}{\partial x} f(p, x)=i \int d p^{\prime} K\left(p^{\prime}-p, x\right) f\left(p^{\prime}, x\right)
$$

where for $V=\frac{1}{2} m \omega^{2} x^{2}$, the kernel simplifies to

$$
i K\left(p^{\prime}-p, x\right)=m \omega^{2} x \frac{\partial}{\partial p} \delta\left(p^{\prime}-p\right)
$$

$f$ therefore must satisfy

$$
\frac{\partial f}{\partial X^{2}}=(m \omega)^{2} \frac{\partial f}{\partial p^{2}} \text {. }
$$

For our discussion of the oscillator, it is useful to express $x$ and $p$ in units of the zero-point motion values $x_{0}$ and $p_{0} . \quad x_{0}^{2}$ is $(2 m \omega)^{-1}$, while $x_{0} p_{0}=\frac{1}{2}$ defines $p_{0}$. These values correspond to the minimum uncertainty character of the ground-state wave function

$$
\psi_{0}(x)=\frac{1}{(2 \pi)^{1 / 4} x_{0}^{1 / 2}} \exp \left(-x^{2} / 4 x_{0}^{2}\right)
$$

From this wave function, we easily compute the Wigner function

$$
f(p, x)=\frac{1}{2 \pi x_{0} p_{0}} \exp \left(-x^{2} / 2 x_{0}^{2}-p^{2} / 2 p_{0}^{2}\right),
$$

which is (not surprisingly) a joint Gaussian in $p, x$. Noting that $(m \omega)^{2}=p_{0}^{2} / x_{0}^{2}$, we write Eq. (2.96) in the form

$$
\frac{\partial f}{\partial\left(x / x_{0}\right)^{2}}=\frac{\partial f}{\partial\left(p / p_{0}^{2}\right)} \text {. }
$$

Clearly the ground-state result $(2.108)$ satisfies this condition, but so does any Gaussian $\exp \left[-A\left(x^{\prime 2}+p^{\prime 2}\right)\right]$, where the prime denotes the natural dimensionless variables $x / x, p / p_{0}$.

Writing the energy in these units,

$$
H(p, x)=\frac{1}{4} \omega\left(p^{\prime 2}+x^{\prime 2}\right) \text {, }
$$

we can easily confirm from Eq. (2.107) that

$$
E_{0}=\int d p d x H(p, x) f(p, x)=\frac{1}{2} \omega,
$$

but the differential equation (2.108) does not determine $f(p, x)$, and so the ground-state energy is correspondingly undetermined.

Next we see that the more general equation (2.83) does give the energy eigenvalues, even though the $f_{m n}$ are not uniquely determined.

Using Eq. (2.104) for the oscillator kernel, we can write the Liouville operator in the form

$$
L=i \omega\left[x^{\prime} \frac{\partial}{\partial p^{\prime}}-p^{\prime} \frac{\partial}{\partial x^{\prime}}\right]
$$

As in the classical case, the Liouville operator acts as a rotation in the phase plane. Naturally, $H$ (or the action, classically) commutes with $L$. Introducing action angle variables in the phase plane as in Fig. 4,

$$
\begin{aligned}
& x=J \cos \varphi, \\
& p=J \sin \varphi,
\end{aligned}
$$

where $J^{2}$ is proportional to $H$, we find

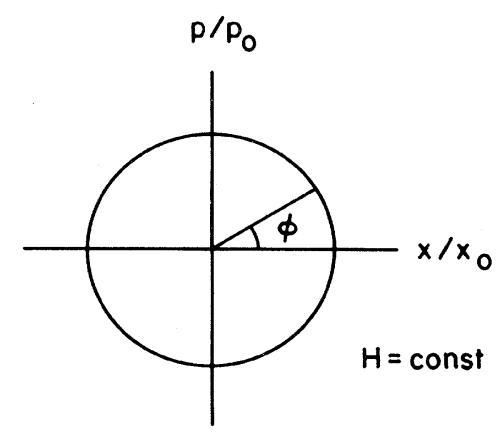

FIG. 4. The harmonic oscillator phase plane. The condition $H=$ const defines a circle in the phase plane, when $p$ and $x$ are normalized to their zero-point values. 


$$
L=i \omega \frac{\partial}{\partial \varphi} .
$$

Hence the single-valued eigenfunctions and eigenvalues are

$$
\begin{aligned}
& f_{n}=e^{-i n \varphi}, \\
& \lambda_{n}=n \omega, \quad n=0, \pm 1, \pm 2, \ldots .
\end{aligned}
$$

These $f_{n}$ may, of course, be multiplied by an arbitrary function of the action $J$, without changing $\lambda_{n}$.

To determine the $f_{m n}$ we may supplement the foregoing with Eq. (2.99). For the oscillator the expansion (2.101) terminates after the second term, giving

$$
\begin{aligned}
{\left[\frac{p^{2}}{2 m}+\frac{m \omega^{2}}{2}-\frac{1}{8 m}\left[\frac{\partial^{2}}{\partial x^{2}}\right.\right.} & \left.\left.+m^{2} \omega^{2} \nabla_{p}^{2}\right]\right] f_{m n} \\
& =\frac{1}{2}\left(E_{m}+E_{n}\right) f_{m n} .
\end{aligned}
$$

Dahl (1981) points out that the equation is identical to the two-dimensional oscillator Schrödinger problem. The solutions are in terms of the generalized Laguerre polynomials $L_{n}^{m}$,

$$
\begin{aligned}
f_{n_{j} n_{i}}= & N_{m_{i} n_{j}} \exp \left(-\frac{1}{2} S\right) \cdot S^{(1 / 2)\left|n_{i}-n_{j}\right|} \cdot L_{n}^{\left|n_{i}-n_{j}\right|}(S) \\
& \times \exp \left[i\left(n_{i}-n_{j}\right) \omega(t-\tau)\right],
\end{aligned}
$$

where $n$ is the greater of $n_{i}, n_{j}$ and the other variables are defined by

$$
\begin{aligned}
& S=\frac{4}{\omega} H_{\text {class }}=\left(p^{\prime 2}+x^{\prime 2}\right), \\
& \tau=-\frac{1}{\omega} \tan ^{-1} \frac{p}{m \omega x}=-\frac{1}{\omega} \tan ^{-1} \frac{p^{\prime}}{x^{\prime}}, \\
& N_{i j}=\frac{1}{\pi} \frac{\left[\frac{n_{j} !}{\left(n_{i} !\right)^{3}}\right]^{1 / 2}}{2} e^{i \delta_{i j}},
\end{aligned}
$$

with $\delta_{i j}$ an arbitrary phase. $x^{\prime}, p^{\prime}$ are the dimensionless variables defined following Eq. (2.100). For the usual choice of phases $e^{i \delta_{i j}}=(-1)^{n_{i}}$. The result (2.116) generalizes earlier results found by Groenewald (1946), Uhlhorn (1956), Heller (1976), and Takabayashi (1954). (Dahl has 2 in place of $1 / \pi$ in $N_{i j}$; we have changed to conform to our convention that $\hbar=1$.)

The ease of obtaining explicit $f_{m n}$ for the onedimensional oscillator allows us to illustrate several further points. First of all, from our previous analysis we know that the functions of $f_{n 0}$ computed from the correct wave functions must be proportional to the eigenfunctions $f_{n} \sim e^{-i n \varphi}$ of the Liouville operator. For example, explicit calculation gives

$$
f_{10}=\left(x^{\prime}-i p^{\prime}\right) f_{00}\left(p^{\prime}, x^{\prime}\right) \propto e^{-i \varphi},
$$

in agreement with Eq. (2.117).

As mentioned earlier, the Wigner distribution for bound states typically becomes negative in some regions of phase space. Let us examine the definition (2.10) for bound states to understand better where negativity will occur. First of all, the variable $R$ has to be in the potential well for $\psi$ to be large; $r$ cannot exceed the range $a$ in the integration. The more highly excited the state, the more nodes in the wave function and the greater the probability of negative values. For $r \sim a$, we can expect significant negative contributions so long as $p<1 / a$, i.e., wavelengths of the order of or larger than the potential range. For sufficiently large $p$, the exponential oscillation restricts $r$ to small values so that the overlap becomes positive. Hence, for $R$ in the potential, we can expect negative values of $f$ whenever $p<1 / a$.

A nice example of these qualitative remarks is given by the first excited state of the oscillator, $\psi_{1} \propto x \psi_{0}(x)$. An elementary calculation yields

$$
f_{11}(p, x)=\frac{1}{2 \pi p_{0} x_{0}}\left(x^{\prime 2}+p^{\prime 2}-1\right) \exp \left(-\frac{1}{2} x^{\prime 2}-\frac{1}{2} p^{\prime 2}\right) .
$$

The circle $x^{\prime 2}+p^{\prime 2}=1$ divides phase space into domains of positivity and negativity. As claimed, we need $x$ in the well $\left(x^{\prime}<1\right)$ and sufficiently small $p$ to reach the negative, nonclassical domain for $f_{11}$. For general $n$, Eq. (2.116) specializes to the simple form

$$
f_{n n}=(-1)^{n} L_{n}\left(p^{\prime 2}+x^{\prime 2}\right) f_{00},
$$

where $L_{n}$ is the ordinary Laguerre polynomial and $f_{00}$ is defined in Eq. (2.107). The domains of negativity are clearly annuli (Fig. 5) in the $x^{\prime} p^{\prime}$ phase plane, since the $L_{n}$

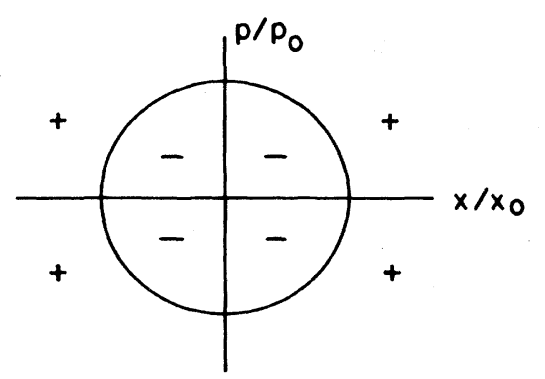

(a)

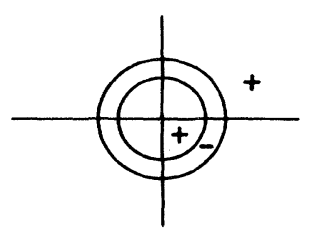

(b)

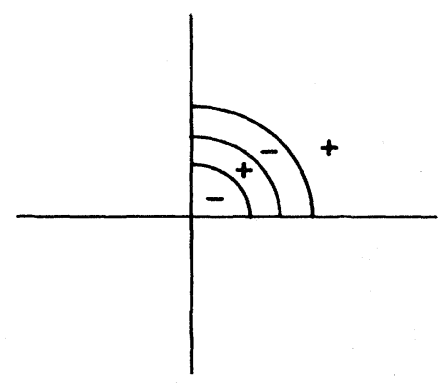

(c)
FIG. 5. Domains of positivity of the Wigner distribution for the first three excited states of the one-dimensional harmonic oscillator. For the first excited state, $f_{11}$ is negative within the unit circle. For the second state, the inner circle is positive, followed by an annulus where $f_{22}$ is negative, and finally a positive region. For large enough action $f_{n n}$ is always positive. The annulus structure reflects the simple properties of the Laguerre polynomial of Eq. (2.120). 
have $n$ real zeros. The signs are such that $f_{n n}$ is positive for large values of the argument.

Finally, we note two especially useful Wigner distributions involving the harmonic oscillator. The first corresponds to the density matrix for thermal equilibrium,

$$
\rho=\exp (-\beta H) / \operatorname{Tr} \exp (-\beta H) \text {. }
$$

The phase-space distribution for this has been computed by Davies and Davies (1975) to be

$$
\begin{aligned}
f(p, x)= & \frac{1}{\pi} \tan h\left(\frac{1}{2} \beta \omega\right) \\
& \times \exp \left[-\frac{1}{2}\left(p^{\prime 2}+x^{\prime 2}\right) \tan h\left(\frac{1}{2} \beta \omega\right)\right] .
\end{aligned}
$$

The second example concerns the coherent state $|\alpha\rangle$ (Glauber, 1963); Carruthers and Nieto, 1965, 1968)

$$
\begin{aligned}
\rho=|\alpha\rangle\langle\alpha|, & \\
|\alpha(t)\rangle & =e^{-(1 / 2)|\alpha|^{2}} \sum_{n=0}^{\infty} \frac{\alpha^{n}(t)}{\sqrt{n} !}|n\rangle, \\
\langle x \mid \alpha\rangle & =\psi_{\alpha}(x) \\
& =\frac{1}{\left(2 \pi x_{0}^{2}\right)^{1 / 4}} \exp \left[-\left(x-\langle x\rangle^{2} / 2 x_{0}^{2}+i\langle p\rangle x\right],\right.
\end{aligned}
$$

where $\alpha(t)=\alpha e^{-i \omega t}$. The phase-space distribution is

$$
\begin{aligned}
& f(p, x, t)=\frac{1}{2 \pi x_{0} p_{0}} \exp \{-[x-x(t)]^{2} / 2 x_{0}^{2} \\
&\left.-[p-p(t)]^{2} / 2 p_{0}^{2}\right\} .
\end{aligned}
$$

Hence $f$ is distributed in a Gaussian manner about the classical motion, in faithful correspondence to the intuitive interpretation of the coherent state.

In each of these cases, which involve summation over the excited oscillator states, $f$ is positive throughout phase space. The negative-valued regions, which are uninterpretable classically, correspond to bound-state problems. The motion of the center of mass, however, is described by an essentially smooth positive phase-space distribution.

Phase-space plots of $f(p, x)$ for the first four energy levels of the one-dimensional square-well potential may be found in the work of Baker, McCarthy, and Porter (1960). In the case of a linear potential, one gets an Airy function, as discussed by Heller (1977) and Dahl (1981).

From these examples and the general discussion, it is clear that bound-state wave functions typically (except for the ground state) give regions of negativity. This is in contrast to the scattering problem, where sufficiently smooth packets give rise to positive joint distributions like the Gaussian packet, Eq. (2.16). In any case, the cross section arises from the positive quantity (2.13). Similarly, the cross section for the production of a many-body bound state is given by an expression like Eq. (2.13), with the internal variables (and negative phase-space regions) integrated out.

\section{G. Hydrodynamic aspects}

It was found very early (Madelung, 1926) that Schrödinger's equation could be recast as a hydrodynamic pair of equations (continuity and an Euler equation for irrotational flow). Writing $\psi=\alpha \exp (i \beta),(\alpha, \beta$ real $)$, we note that the probability current is

$$
\underline{j}=\alpha^{2} \nabla \beta / m \text {, }
$$

so that we can identify the velocity as

$$
\underline{u}=\nabla \beta / m \text {. }
$$

This suggests that $\beta / m$ plays the role of a velocity potential $\varphi$.

The same current, obeying the usual conservation law,

$$
\frac{\partial n}{\partial t}+\nabla \cdot \underline{j}=0
$$

$\left(n=\alpha^{2}\right.$ ), is obtained as the imaginary part of the Schrödinger equation in the $(\alpha, \beta)$ variables. The real part can be identified with the (irrotational) Euler equation.

We now explore the hydrodynamical connection in the context of the Wigner distribution, beginning with the equation of motion (2.26).

Integration over $d^{3} p$ gives immediately

$$
\frac{\partial n}{\partial t}+\nabla \cdot(n u)=0,
$$

where local average velocity $\underline{u}(R, t)$ is defined by

$$
\underline{u}(R, t)=\frac{\left.\int d^{3} p \underline{v}_{p} f(\underline{p}, \underline{R}), t\right)}{\int d^{3} p f(\underline{p}, \underline{R}, t)}
$$

$\left(v_{p}=\underline{p} / m\right)$. The potential term disappears, since $\int d^{3} p$ produces a $\delta(\underline{r})$ under the $d^{3} r$ integral.

The analog of Newton's law follows on taking the first moment

$$
n u_{i}=\int d^{3} p v_{p_{i}} f(\underline{p}, \underline{R}, t)
$$

in the equation of motion,

$$
\begin{aligned}
& \frac{\partial}{\partial t}\left(n u_{i}\right)+\frac{\partial}{\partial x_{j}} \int d^{3} p v_{p_{i}} v_{p_{j}} f(p, R, t) \\
& =\int d^{3} p d^{3} p^{\prime} K\left(p^{\prime}-p, R\right) v_{p_{i}} f\left(p^{\prime}, R, t\right) \\
& \quad=-\frac{1}{m}\left(\nabla_{i} V\right) n,
\end{aligned}
$$

the latter form following on using the explicit form for $K$.

We compare this with the usual momentum equation of continuum hydrodynamics,

$$
\begin{aligned}
& \frac{\partial}{\partial t}\left(\rho u_{i}\right)+\frac{\partial}{\partial x_{k}} T_{i k}=0, \\
& T_{i k}=\rho u_{i} u_{k}+\delta_{i k} p,
\end{aligned}
$$

where $\rho$ is the mass density and $p$ the pressure. We now see that the stress tensor for the Schrödinger equation is

$$
\begin{aligned}
& T_{i k}^{s}=\rho\left\langle v_{i} v_{k}\right\rangle+\delta_{i j} p^{s}, \\
& \nabla p^{s} \equiv n \nabla V, \\
& \left\langle v_{i} v_{k}\right\rangle \equiv \frac{\int d^{3} p v_{p_{j}} f(p, R, t)}{\int d^{3} p f(p, R, t)} .
\end{aligned}
$$


Equation (2.136) involves the correlation $\left\langle v_{i} v_{k}\right\rangle$, in contrast to the uncorrelated velocities $u_{i} u_{k}$ in Eq. (2.133). By reexpressing (2.136) in terms of uncorrelated velocities, we make contact with the usual Euler equation.

Making use of the identity

$$
\left\langle v_{i} v_{k}\right\rangle \equiv\left\langle\left(v_{i}-u_{i}\right)\left(v_{k}-u_{k}\right)\right\rangle+u_{i} u_{k}
$$

we rewrite (2.131) as

$$
\frac{\partial}{\partial t}\left(\rho u_{i}\right)+\frac{\partial}{\partial x_{k}}\left(\rho u_{i} u_{k}\right)=-n \nabla V-\frac{\partial}{\partial x_{k}} p_{i j},
$$

where the kinetic pressure tensor is given by

$$
p_{i k} \equiv \rho \frac{\int d^{3} p\left(v_{i}-u_{i}\right)\left(v_{k}-u_{k}\right) f(p, R, t)}{\int d^{3} p f(p, R, t)} .
$$

Expanding derivatives on the left-hand side and using current conservation reduces Eq. (2.138) to Euler's equation

$$
\rho\left(\frac{\partial u_{i}}{\partial t}+\underline{u} \cdot \nabla u_{i}\right)=-\rho \frac{\nabla_{i} V}{m}-\frac{\partial}{\partial x_{j}} p_{i j},
$$

when $-\rho \nabla_{i} V / m$ is the external force density. Similar considerations have been discussed by Kan and Griffin (1977).

\section{H. Effect of an external potential}

Our discussion so far has dealt with pure scattering or bound-state problems. In the presence of a timedependent external potential, we can excite bound states (either to other bound states or to the continuum) or can induce "capture" of the free particle into a bound state. In principle, these processes are symmetrical, but the usual experimental arrangement leads to a somewhat different treatment.

Corresponding to the choice of potential energy,

$$
V(R)+V_{\mathrm{ex}}(R, t),
$$

we write the basic equation of motion as

$$
\begin{gathered}
{\left[\frac{\partial}{\partial t}+v \cdot \nabla\right] f(p, R, t)-\int d^{3} p^{\prime} K\left(p^{\prime}-p, R\right) f\left(p^{\prime}, R, t\right)} \\
=\int d^{3} p^{\prime} K_{\mathrm{ex}}\left(p^{\prime}-p, R, t\right) f\left(p^{\prime}, R, t\right) .
\end{gathered}
$$

For an initial bound state, Eq. (2.44) no longer holds, since $f_{B}$ does not obey the homogeneous equation, but instead

$$
\frac{D}{D t} f_{B}\left(p^{\prime}, R, t\right)-\int d^{3} p^{\prime} K\left(p^{\prime}-p, R\right) f_{B}\left(p^{\prime}, R, t\right)=0 .
$$

Of course, the time dependence actually goes away, as discussed in Sec. II.F. In addition, Eq. (2.143) does not determine $f_{B}$.

In order to solve Eq. (2.142), we clearly need the interacting Green's function

$$
\begin{array}{r}
i \frac{D}{D t} G(p, R, t)-i \int d^{3} p^{\prime} K\left(p^{\prime}-p, R\right) G\left(p^{\prime}, R, t\right) \\
=\delta(\underline{p}) \delta(\underline{R}) \delta(t)
\end{array}
$$

subject to suitable boundary conditions. For example, the Wigner function for an initial bound state satisfies

$$
f(p, R, t)=f_{B}+\int_{-\infty}^{t} d t^{\prime} \int d^{3} p^{\prime} \int d^{3} R^{\prime} G_{\mathrm{ret}}\left(p^{\prime}-p, R^{\prime}, t^{\prime}\right) K_{\mathrm{ex}}\left(p^{\prime}-p, R^{\prime}, t^{\prime}\right) f\left(p^{\prime}, R^{\prime}, t^{\prime}\right) .
$$

If the initial particle is free, we can still use Eq. (2.54) with $K+K_{\text {ex }}$ replacing $K$. In this case, the final state will contain both bound-state and continuum components. Since the wave function can be written as an expansion in energy eigenfunctions as

$$
\psi=\sum_{B} \alpha_{B} \psi_{B}+\int d^{3} k C(k) \psi_{k}^{+},
$$

only energy-degenerate cross terms survive in the timeaveraged Wigner function. In particular, the contribution of the final bound state $B$ (assuming no accidental degeneracy) is $\left|\alpha_{B}\right|^{2} f_{B B}$, as expected.

The explicit calculation of the retarded Green's function obeying Eq. (2.144) is usually quite complicated. We content ourselves with expressing $G$ in terms of the "eigenmatrices" $f_{m n}$ of the Liouville operator [of Eq. (2.93)]. Introducing the Fourier transform $G(p, R, \omega)$ obeying

$$
(L-\omega) G(p, R, \omega)=\delta(\underline{p}) \delta(\underline{R}),
$$

and expanding $G=\sum G_{m n} f_{m n}$ gives

$$
\sum_{m n} C_{m n}\left(\lambda_{m n}-\omega\right) f_{m n}(p, R)=\delta(p) \delta(R) \text {. }
$$

The orthogonality relation (2.93) is now used to evaluate $C_{m n}$, giving

$$
G_{R}(\underline{p}, \underline{R}, \omega)=\sum_{m n} \frac{f_{m n}^{*}(0,0) f_{m n}(p, R)}{\lambda_{m n}-\omega+i \varepsilon}
$$

when the $i \varepsilon$ is added to enforce the right boundary condition in the continuum.

Many commonly occurring problems, e.g., excitation through interaction with an external system, are conveniently modeled by the introduction of an external potential energy $V_{\mathrm{ex}}(\underline{x}, t)$. The total Hamiltonian is then $H_{T}=T+V+V_{\text {ex }}$, and it is natural to express the evolution of the phase-space distribution in terms of the $f_{m n}$ referred to the Hamiltonian $T+V$ of the system being perturbed. We therefore write the expansion

$$
f(t)=\sum_{m n} C_{m n}(t) f_{m n}(t),
$$

where the $C_{m n}$ depend on time by virtue of $V_{\mathrm{ex}}$. The $f_{m n}$ form a complete set, though the orthonormality relations are not very simple.

The expansion coefficients are exactly those of the density matrix. For a pure state, we have 


$$
\begin{aligned}
& \rho=|\psi\rangle\langle\psi| \\
& =\sum_{m, n} a_{m} a_{n}^{*} \rho_{m n} \equiv \sum_{m n} C_{m n} \rho_{m n}, \\
& |\psi\rangle=\sum_{m} \alpha_{m}|m\rangle,
\end{aligned}
$$

where $\rho_{m n} \equiv|m\rangle\langle n|$ is the same as occurred in Eq. (2.56).

Beginning with this result, one can do time-dependent perturbation theory in the standard manner by writing

$$
C_{m n}=C_{m n}^{(0)}+\lambda C_{m n}^{(1)}+\lambda^{2} C_{m n}^{(2)}+\cdots
$$

(where $V_{\text {ex }}$ is of order $\lambda$ ). We then obtain the set of coupled iterative equations

$$
\begin{aligned}
& i \dot{C}^{(0)}=0, \\
& i \dot{C}_{m n}^{(1)}=\sum_{k}\left(\widetilde{V}_{m k} C_{k n}^{(0)}-\widetilde{V}_{k n} C_{m k}^{(0)}\right), \\
& i \dot{C}_{m n}^{(2)}=\sum_{k}\left(\widetilde{V}_{m k} C_{k m}^{(1)}-\widetilde{V}_{k m} C_{m k}^{(1)}\right) .
\end{aligned}
$$

By normalization the diagonal C's must obey the constraint

$$
\sum_{m} C_{m m}=1
$$

These results can be applied to nondegenerate groundstate excitation in the standard way. For $\lambda=0$, $C_{m n}^{(0)}=\delta_{m 0} \delta_{n 0}$. If $t<0, V_{\mathrm{ex}}=0$ and $C_{m n}^{(i)}(t<0)=0$. The $C_{m n}^{(0)}$ remain constant in time.

\section{Gauge invariance}

In the presence of external electromagnetic potentials, it is necessary to modify the definition (2.10) in order to obtain a gauge-invariant phase-space distribution function. Under a gauge transformation the potentials transform like

$$
A^{\prime}=A+\nabla \chi, \varphi^{\prime}=\varphi-\frac{1}{c} \frac{\partial \chi}{\partial t} .
$$

The form of the Schrödinger equation is unchanged when the wave function transforms as

$$
\psi^{\prime}=\psi e^{i e \chi / \hbar}
$$

From this it is clear that the integrand of Eq. (2.10) requires modification by a path-dependent phase factor

$$
\begin{aligned}
f_{P}(p, R, t)=\int & \frac{d^{3} r}{(2 \pi)^{3}} e^{-i \underline{p} \cdot r} \\
& \times \psi^{*}\left(R-\frac{1}{2} r, t\right) \psi\left(R+\frac{1}{2} r, t\right) \\
& \times \exp \left[\int_{P} \mathbf{A} \cdot \mathrm{d} l\right],
\end{aligned}
$$

where the line integral runs along a path $P$ beginning at $\underline{R}-\frac{1}{2} \underline{r}$ and ending at $\underline{R}+\frac{1}{2} \underline{r}$.

In a simply connected region, the loop integral of $\mathbf{A}$ will vanish and the particular path $P$ makes no difference.
For multiply connected domains, such as are encountered in the Aharonov-Bohm experiment, the phase factor becomes crucial.

The distribution (2.157) has an equation of motion whose classical limit is the one-particle Liouville equation (2.28) when the force term $-\nabla V$ is replaced by the usual Lorentz force (de Groot and Suttorp, 1972; BialynickiBirula, 1977).

\section{J. Spin variables}

We note briefly the extensions required to describe the spin variable in the most important case of spin $\frac{1}{2}$. Letting $a, b$ denote the $z$ component of the spin, we define a $2 \times 2$ matrix Wigner function $f$ with elements

$$
\begin{aligned}
f_{a b}(p, R, t)=\int & \frac{d^{3} r}{(2 \pi)^{3}} e^{-i \underline{p} \cdot r} \psi_{b}^{*}\left(R-\frac{1}{2} r, t\right) \\
& \times \psi_{a}\left(R+\frac{1}{2} r, t\right) .
\end{aligned}
$$

The ordinary density is then

$$
\begin{aligned}
f(p, R, t) & =\operatorname{Trf} \\
& =\int \frac{d^{3} r}{(2 \pi)^{3}} e^{-i \underline{p} \cdot r} \psi^{*}\left(R-\frac{1}{2} r, t\right) \psi\left(R+\frac{1}{2} r, t\right) .
\end{aligned}
$$

The reality of $f$ for the spinless case is now replaced by Hermiticity $\mathbf{f}^{+}=\mathbf{f}$, i.e.,

$$
f_{a b}^{*}=f_{b a} \text {. }
$$

Such an $f$ can be represented by two independent functions $f_{0}$ and $f_{1}$ and a real unit vector $\hat{n}$ as follows:

$$
\mathbf{f}=f_{0}+\underline{\sigma} \cdot \hat{n} f_{1} \text {. }
$$

The polarization density can be computed from the expectation value of the spin operator as follows:

$$
\begin{aligned}
\underline{\underline{\sigma}}(\underline{p}, \underline{R}, t) & =\operatorname{Tr}(\underline{\sigma} \mathbf{f}) \\
& =\int \frac{d^{3} r}{(2 \pi)^{3}} e^{-i \underline{p} \cdot r} \psi^{\dagger}\left(R-\frac{1}{2} r, t\right) \underline{\sigma} \psi\left(R+\frac{1}{2} r, t\right) .
\end{aligned}
$$

We can express the ordinary and spin densities in terms of $f_{0}$ and $f_{1}$ as follows:

$$
\begin{aligned}
& f(\underline{p}, \underline{R}, t)=2 f_{0}(p, R, t), \\
& \underline{\sigma}(\underline{p}, \underline{R}, t)=2 \hat{n} f_{1}(\underline{p}, \underline{R}, t) .
\end{aligned}
$$

\section{THE NONRELATIVISTIC TWO-BODY PROBLEM}

\section{A. Definitions}

The two-body problem exhibits in simplified form some of the kinematical features of the $N$-body system. Of 
course, one recovers the one-body problem in the absence of external potentials when the two-body potential depends on the distance between the particles. Until the final parts of this section, we treat the problem of two spinless, distinguishable particles of the same mass, with Hamiltonian

$$
H=\frac{p_{1}^{2}}{2 m}+\frac{p_{2}^{2}}{2 m}+v\left(\underline{x}_{1}-\underline{x}_{2}\right)+V_{1}\left(\underline{x}_{1}, t\right)+V_{2}\left(\underline{x}_{2}, t\right) .
$$

The external potentials $V_{i}$ act on the $i$ th particle. We have in mind two classes of problems:

(1) scattering of particles 1 and 2,

(2) breakup of a bound state by external potentials.

The $V_{i}$ are imagined to have a finite extent in space. An example of case (2) is the photodisintegration of the deuteron, where particle 1 is a proton, particle 2 a deuteron, while $V_{1}$ represents an external electric potential and $V_{2}$ vanishes.

In order to generalize Eq. (2.10), we again take the Fourier transform on the relative coordinates $x_{1}^{\prime}-x_{1}$, $x_{2}^{\prime}-x_{2}$ in the density matrix $\Psi^{*}\left(x_{1}, x_{2}\right) \Psi\left(x_{1}^{\prime}, x_{2}^{\prime}\right)$. Making the definitions

$$
\begin{aligned}
& \underline{x}_{1} \equiv \underline{R}_{1}-\frac{1}{2} r_{1}, \quad \underline{x}_{1}^{\prime}=\underline{R}_{1}+\frac{1}{2} \underline{r}_{1}, \\
& \underline{x}_{2} \equiv \underline{R}_{2}-\frac{1}{2} \underline{r}_{2}, \quad \underline{x}_{2}^{\prime}=\underline{R}_{2}+\frac{1}{2} \underline{r}_{2},
\end{aligned}
$$

we define the two-particle distribution to be

$$
f_{2}\left(\underline{p}_{1}, \underline{R}_{1}, \underline{p}_{2}, \underline{R}_{2}, t\right)
$$

$$
\begin{aligned}
=\int & \frac{d^{3} r_{1} d^{3} r_{2}}{(2 \pi)^{6}} e^{-i \underline{p_{1}} \underline{r}_{1}-i \underline{p}_{2} \cdot \underline{\underline{r}}_{2}} \Psi^{*}\left(\underline{R}_{1}-\frac{1}{2} \underline{\boldsymbol{r}}_{1}, \underline{R}_{2}-\frac{1}{2} \underline{r}_{2}, t\right) \\
& \times \Psi\left(\underline{R}_{1}+\frac{1}{2} \underline{r}_{1}, \underline{R}_{2}+\frac{1}{2} \underline{\underline{r}}_{2}, t\right) .
\end{aligned}
$$

Expanding in momentum basis, we have the alternative form

$$
\begin{aligned}
& f_{2}\left(\underline{p}_{1}, \underline{R}_{1}, \underline{p}_{2}, \underline{R}_{2}, t\right) \\
&=\int \frac{d^{3} q_{1} d^{3} q_{2}}{(2 \pi)^{6}} e^{i \underline{q}_{1} \cdot \underline{R}_{1}+i \underline{q}_{2} \cdot \underline{R}_{2}} C^{*}\left(\underline{p}_{1}-\frac{1}{2} \underline{q}_{1}, \underline{p}_{2}-\frac{1}{2} \underline{q}_{2}, t\right) \\
& \quad \times C\left(\underline{p}_{1}+\frac{1}{2} \underline{q}_{1}, \underline{p}_{2}+\frac{1}{2} \underline{q}_{2}, t\right) .
\end{aligned}
$$

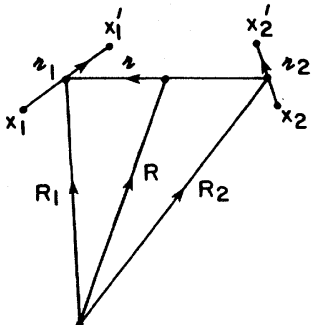

FIG. 6. Kinematical space variables suitable for the twoparticle distribution [see Eq. (3.3)]. The $x_{i}, x_{i}^{\prime}$ label the split points of the wave function. $R_{1}, R_{2}$ turn out to be the effective locations of particles 1 and 2, while $R=\frac{1}{2}\left(R_{1}+R_{2}\right)$ and $r=R_{1}-R_{2}$ have the usual significance of total and relative coordinates.

Although $f_{2}$ itself need not be positive definite, the joint momentum and coordinate distributions are, as expected,

$$
\begin{aligned}
& \int d^{3} p_{1} d^{3} p_{2} f_{2}\left(\underline{p}_{1} \underline{R}_{1}, \underline{P}_{2} \underline{p}_{2} t\right)=\left|\Psi\left(\underline{R}_{1}, \underline{R}_{2} t\right)\right|^{2}, \\
& \int d^{3} R_{1} d^{3} R_{2} f_{2}\left(\underline{p}_{1} \underline{R}_{1}, \underline{p}_{2} \underline{R}_{2} t\right)=\left|C\left(\underline{p}_{1}, \underline{p}_{2}, t\right)\right|^{2} .
\end{aligned}
$$

As suggested by the notation, $\left(p_{i}, R_{i}\right)$ have the significance of the position and momentum variables in the kinematical phase space.

Our analysis will require additional position variables defined by Fig. 6:

$$
\begin{aligned}
& \underline{R}=\frac{1}{2}\left(\underline{R}_{1}+\underline{R}_{2}\right), \quad \underline{r}=\underline{R}_{1}-\underline{R}_{2}, \\
& \underline{\rho}=\underline{r}_{1}-\underline{r}_{2}, \tilde{R}=\frac{1}{2}\left(\underline{r}_{1}+\underline{r}_{2}\right), \\
& \underline{x}_{1}-\underline{x}_{2}=\underline{r}-\frac{1}{2} \rho, \underline{x}_{1}^{\prime}-\underline{x}_{2}^{\prime}=\underline{r}+\frac{1}{2} \underline{\rho} .
\end{aligned}
$$

As suggested by the notation, $(R, r)$ are the $\mathrm{cm}$ and relative coordinates. In addition, we shall require total and relative momenta,

$$
\begin{aligned}
& \underline{p}=\underline{p}_{1}+\underline{p}_{2}, \quad \underline{p}=\frac{1}{2}\left(\underline{p}_{1}-\underline{p}_{2}\right), \\
& \frac{p_{1}^{2}}{2 m}+\frac{p_{2}^{2}}{2 m}=\frac{p^{2}}{2 \mu} \frac{+P^{2}}{2 M} \quad\left(\mu=\frac{1}{2} m, M=2 m\right) .
\end{aligned}
$$

The two-particle Boltzmann drift term can then be expressed as

$$
\begin{aligned}
& \underline{v}_{1} \cdot \nabla_{R_{1}}+\underline{v}_{2} \cdot \nabla_{R_{2}}=V \cdot \nabla_{R}+v \cdot \nabla_{r}, \\
& v=\left(p_{1}-p_{2}\right) / m, \quad V=\underline{P} / M .
\end{aligned}
$$

A similar decomposition can be made for particles of unequal mass.

\section{B. Equation of motion}

The equation of motion for $f_{2}$ is derived in direct analogy to the one-particle case. We find

$$
\begin{aligned}
\left(\frac{\partial}{\partial t}+\underline{v}_{1} \cdot \nabla_{1}+\underline{v}_{2} \cdot \nabla_{2}\right) f_{2}\left(\underline{p}_{1}, \underline{R}_{1} \underline{p}_{2} \underline{R}_{2} t\right)= & \int d^{3} p_{1}^{\prime} d^{3} p_{2}^{\prime} K_{2}\left(\underline{p}_{1}^{\prime}-\underline{p}_{1} \underline{p}_{2}^{\prime}-\underline{p}_{2}, \underline{R}_{1} \underline{R}_{2}\right) f_{2}\left(p_{1}^{\prime} \underline{R}_{1} p_{2}^{\prime} \underline{R}_{2} t\right) \\
& +\int d^{3} p_{1}^{\prime} K_{1}\left(p_{1}^{\prime}-\underline{p}_{1}, \underline{R}_{1}, t\right) f_{2}\left(\underline{p}_{1}^{\prime} \underline{R}_{1} \underline{p}_{2} \underline{R}_{2} t\right) \\
& +\int d^{3} p_{2}^{\prime} K_{1}\left(\underline{p}_{2}^{\prime}-\underline{p}_{2}, \underline{R}_{2} t\right) f_{2}\left(\underline{p}_{1} \underline{R}_{1} \underline{p}_{2}^{\prime} \underline{R}_{2} t\right),
\end{aligned}
$$


where $v_{1}$ is $p_{1} / m, \nabla_{1}$ is $\partial / \partial \mathbf{R}_{1}$, etc. The kernels $K_{1}$ are defined exactly as in the one-body problem except that the potentials $V_{1,2}$ can be time dependent. The kernel $K_{2}$ comes from the interparticle potential

$$
i K_{2}\left(\underline{p}_{1}^{\prime}-\underline{p}_{1}, \underline{p}_{2}^{\prime}-\underline{p}_{2}, \underline{R}_{1}, \underline{R}_{2}\right) \equiv \int \prod_{j=1}^{2} \frac{d^{3} r_{j}}{(2 \pi)^{3}} e^{i\left(\underline{p}_{j}^{\prime}-\underline{p}_{j}\right) \cdot r_{j}}\left[v\left(\underline{R}_{1}+\frac{1}{2} \underline{r}_{1}, \underline{R}_{2}+\frac{1}{2} \underline{r}_{2}\right)-v\left(\underline{R}_{1}-\frac{1}{2} \underline{r}_{2} \underline{R}_{2}-\frac{1}{2} \underline{r}_{2}\right)\right] .
$$
find

$K_{2}$ simplifies greatly by virtue of $v$ depending only on the relative coordinate. After a suitable change of variables, we

$$
\begin{aligned}
i K_{2}\left(p_{1}^{\prime}-p_{1}, p_{2}^{\prime}-p_{2}, R_{1}, R_{2}\right) & =\int \frac{d^{3} \widetilde{R} d^{3} \rho}{(2 \pi)^{6}} \exp \left[i\left(p_{1}^{\prime}-p_{1}\right) \cdot\left(\widetilde{R}+\frac{1}{2} \rho\right)+i\left(p_{2}^{\prime}-p_{2}\right) \cdot\left(\widetilde{R}-\frac{1}{2} \rho\right)\right]\left[v\left(r+\frac{1}{2} \rho\right)-v\left(r-\frac{1}{2} \rho\right)\right] \\
& =\delta\left(p_{1}^{\prime}+p_{2}^{\prime}-p_{1}-p_{2}\right) \int \frac{d^{3} \rho}{(2 \pi)^{3}} \exp \left[i\left(p^{\prime}-p\right) \cdot \rho\right]\left[v\left(r+\frac{1}{2} \rho\right)-v\left(r-\frac{1}{2} \rho\right)\right] \\
& =\delta\left(p_{1}^{\prime}+p_{2}^{\prime}-p_{1}-p_{2}\right) i K_{1}\left(p^{\prime}-p, r\right)
\end{aligned}
$$

which is independent of $R$. The kernel conserves overall momentum (an expression of translation invariance), while the action of the potential is determined by the relative variables. In the presence of external potentials, the overall space-time translation invariance is lost, and energy and momentum can be exchanged with the two-body system.

We now demonstrate in detail the separation of the center-of-mass and relative motion starting from Eq. (3.9). The assumed wave function is

$$
\Psi=\chi\left[\frac{\underline{x}_{1}+\underline{x}_{2}}{2}, t\right] \Phi\left(\underline{x}_{1}-\underline{x}_{2}, t\right) .
$$

$\chi$ is a wave packet representing the center-of-mass motion of the form of Eq. (2.2) with $C$ as in Eq. (2.14), and with $P$ the total $\mathrm{cm}$ momentum peaked around $P_{0}$. An elementary calculations leads to the phase-space distribution

$f_{2}\left(\underline{p}_{1} \underline{R}_{1}, \underline{P}_{2} \underline{R}_{2} t\right)$

$$
=f_{0}\left[\underline{p}_{1}+\underline{p}_{2} \frac{\underline{R}_{1}+\underline{R}_{2}}{2}, t\right] f\left(\frac{\underline{p}_{1}-\underline{p}_{2}}{2}, R_{1}-R_{2}, t\right] \text {, }
$$

where $f_{0}$ is the free-packet function of Eq. (2.14) and $f$, given by

$$
f(\underline{p}, \underline{r}, t)=\int \frac{d^{3} \rho}{(2 \pi)^{3}} e^{-i \underline{p} \cdot} \cdot \underline{\Phi} *\left(\underline{r}-\frac{1}{2} \rho, t\right) \Phi\left(\underline{r}+\frac{1}{2} \underline{\rho}, t\right),
$$

is time independent for the case of an energy eigenstate. Note that $f_{0}$ is a function of $\underline{R}-\underline{V} t$, as expected. Therefore, $\partial / \partial t+V \cdot \nabla_{R}$ gives zero when acting on $f_{0}$, so that the left-hand side of Eq. (3.9) becomes

$$
f_{0}(\underline{P}, \underline{R}, t)\left[\frac{\partial}{\partial t}+\underline{v} \cdot \nabla_{r}\right] f(\underline{p}, \underline{r}, t) .
$$

To see that $f_{0}$ also factors out of the right-hand side, we use Eq. (3.10) to simplify. Noting that the Jacobian is unity on going from $\left(p_{1}, p_{2}\right) \rightarrow(\underline{P}, p)$, we find

$$
\begin{aligned}
\int \frac{d^{3} P^{\prime} d^{3} p^{\prime}}{(2 \pi)^{3}} \delta & \left(P^{\prime}-P\right) K_{1}\left(p^{\prime}-p, r\right) f_{0}\left(P^{\prime}, R, t\right) f\left(p^{\prime}, r, t\right) \\
& =f_{0}(P, R, t) \int \frac{d^{3} p^{\prime}}{(2 \pi)^{3}} K_{1}\left(p^{\prime}-p, r\right) f\left(p^{\prime}, r, t\right) .
\end{aligned}
$$

Hence the relative motion is described by the one-body equation of motion with $K$, and calculated from the potential $v(r)$. Note that the factorization occurs independently of the shape of the $\mathrm{cm}$ wave packet.

\section{Technical interlude: free $\mathbf{N}$-particle retarded Green's functions}

In order to cast the equation of motion in integral form suitable for scattering problems, we need the generalization of the retarded Green's function to two and more particles.

Consider the defining equation for $N$ particles,

$$
\frac{D G}{D t}\left(\underline{p}_{1} \underline{R}_{1} \cdots \underline{p}_{N} \underline{R}_{N} t\right)=\delta\left(\underline{R}_{1}\right) \delta\left(\underline{R}_{2}\right) \cdots \delta\left(\underline{R}_{N}\right) \delta(t),
$$

$$
\frac{D}{D t}=\frac{\partial}{\partial t}+\sum_{i=1}^{N} \underline{v}_{i} \cdot \nabla_{i}
$$

Writing $\boldsymbol{G}$ as a Fourier transform,

$$
\begin{aligned}
G\left(\underline{p}_{1} \underline{R}_{1} \cdots \underline{p}_{N} \underline{R}_{N} t\right)=\int \prod_{j=1}^{N} \frac{d^{3} \underline{q}_{i}}{(2 \pi)^{3}} \\
\times e^{i \underline{q}_{j} \cdot \underline{R}_{j}} G\left(\underline{p}_{1} \underline{q}_{1} \cdots \underline{p}_{N} \underline{q}_{N} t\right),
\end{aligned}
$$

gives the equation

$$
\left(i \frac{\partial}{\partial t}-\sum_{j=1}^{N} \underline{q}_{j} \cdot \underline{v}_{j}\right] G\left(\underline{p}_{1} \underline{q}_{1} \cdots \underline{p}_{N} \underline{q}_{N} t\right)=i \delta(t) .
$$

Finally, transforming in time gives 


$$
\boldsymbol{G}\left(\underline{p}_{1} \underline{q}_{1} \cdots \underline{p}_{N} \underline{q}_{N} \omega\right)=\frac{i}{\omega+i \epsilon-\sum_{j} \underline{q}_{j} \cdot \underline{v}_{j}},
$$

where the $i \varepsilon$ has been added to enforce a retarded boundary condition.

In $R, t$ variables, we find easily

$$
\begin{aligned}
G_{R}\left(\underline{p}_{1} \underline{R}_{1} \cdots\right. & \left.\underline{p}_{N} \underline{R}_{N} t\right) \\
& =\vartheta(t) \delta\left(\underline{R}_{1}-\underline{v}_{1} t\right) \delta\left(\underline{R}_{2}-\underline{v}_{2} t\right) \cdots \delta\left(\underline{R}_{N}-\underline{v}_{N} t\right) .
\end{aligned}
$$

an intuitive result whose correctness can be verified directly.

In passing, we note another intuitive but important result,

$$
\frac{D}{D t} F\left(\underline{R}_{i}-\underline{v}_{i} t\right)=0
$$

$$
\begin{aligned}
& i K_{1}\left(p^{\prime}-p, q, t\right)=V(q, t)\left[\delta\left(p-p^{\prime}-\frac{1}{2} q\right)-\delta\left(p-p^{\prime}+\frac{1}{2} q\right)\right] \\
& i K_{2}\left(p_{1}^{\prime}-p_{1}, p_{2}^{\prime}-p_{2}, q_{1}, q_{2}\right)=v\left(q_{1}, q_{2}\right)\left[\delta\left(p_{1}-p_{1}^{\prime}-\frac{1}{2} q_{1}\right) \delta\left(p_{2}-p_{2}^{\prime}-\frac{1}{2} q_{2}\right)-\delta\left(p_{1}-p_{1}^{\prime}+\frac{1}{2} q_{1}\right) \delta\left(p_{2}-p_{2}^{\prime}+\frac{1}{2} q_{2}\right)\right] .
\end{aligned}
$$

In the usual case where $v$ depends on the relative coordinate, we find

$$
v\left(\underline{q}_{1}, \underline{q}_{2}\right)=(2 \pi)^{3} \delta\left(\underline{q}_{1}+\underline{q}_{2}\right) v\left(\frac{\underline{q}_{1}-\underline{q}_{2}}{2}\right) .
$$

From now on, we write our equations for this case only.

The equation of motion can be written as

$$
\begin{aligned}
\int i \frac{\partial}{\partial t}- & \left.q_{1} \cdot v_{1}-q_{2} \cdot v_{2}\right] f_{2}\left(p_{1} q_{1} p_{2} q_{2} t\right) \\
& =\int \frac{d^{3} q_{1}^{\prime}}{(2 \pi)^{3}} v\left(q_{1}^{\prime}\right)\left[f_{2}\left(p_{1}-\frac{1}{2} q_{1}^{\prime}, q_{1}-q_{1}^{\prime}, p_{2}+\frac{1}{2} q_{1}^{\prime}, q_{2}-q_{1}^{\prime}, t\right)-f_{2}\left(p_{1}+\frac{1}{2} q_{1}^{\prime}, q_{1}-q_{1}^{\prime}, p_{2}-\frac{1}{2} q_{1}^{\prime}, q_{2}+q_{1}^{\prime}, t\right)\right] \\
& +\int \frac{d^{3} q_{1}^{\prime}}{(2 \pi)^{3}} V\left(q_{1}^{\prime}, t\right)\left[f_{2}\left(p_{1}-\frac{1}{2} q_{1}^{\prime}, q_{1}-q_{1}^{\prime}, p_{2} q_{2} t\right)-f_{2}\left(p_{1}+\frac{1}{2} q_{1}^{\prime}, q_{1}-q_{1}^{\prime}, p_{2} q_{2} t\right)\right] \\
& +\int \frac{d^{3} q_{2}^{\prime}}{(2 \pi)^{3}} V\left(q_{2}^{\prime}, t\right)\left[f_{2}\left(p_{1} q_{1}, p_{2}-\frac{1}{2} q_{2}, q_{2}-q_{2}^{\prime} t\right)-f_{2}\left(p_{1} q_{1}, p_{2}+\frac{1}{2} q_{2}, q_{2}-q_{2}^{\prime} t\right)\right] .
\end{aligned}
$$

It is also of occasional interest to know the equation for the reduced distribution function for (say) particle 1 , in which one does not care what particle 2 is doing. The definition is

$$
f^{(1)}\left(p_{1} R_{1} t\right) \equiv \int d^{3} p_{2} d^{3} R_{2} f_{2}\left(p_{1} R_{1} p_{2} R_{2} t\right)
$$

Note that the $\int d^{3} R_{2}$ integration simply amounts to going to the limit $q_{2} \rightarrow 0$. The equation of motion for $f^{(1)}$ is

$$
\begin{aligned}
{\left[i \frac{\partial}{\partial t}-v_{1} \cdot q_{1}\right] f^{(1)}\left(p_{1} q_{1} t\right)=} & \int \frac{d^{3} q_{1}^{\prime}}{(2 \pi)^{3}} v\left(q_{1}^{\prime} \int d^{3} p_{2}\left[f_{2}\left(p_{1}-\frac{1}{2} q_{1}^{\prime}, q_{1}-q_{1}^{\prime}, p_{2}, q_{1}^{\prime} t\right)-f_{2}\left(p_{1}+\frac{1}{2} q_{1}^{\prime}, q_{1}-q_{1}^{\prime}, p_{2}, q_{1}^{\prime}, t\right)\right]\right. \\
& +\int \frac{d^{3} q_{1}^{\prime}}{(2 \pi)^{3}} V\left(q_{1}^{\prime}, t\right)\left[f^{(1)}\left(p_{1}-\frac{1}{2} q_{1}^{\prime}, q_{1}-q_{1}^{\prime}, t\right)-f^{(1)}\left(p_{1}+\frac{1}{2} q_{1}^{\prime}, q_{1}-q_{1}^{\prime}, t\right)\right]
\end{aligned}
$$

A similar equation exists for particle 2. In deriving this equation, we have shifted variables in the $p_{2}$ integration to remove the apparent $q_{1}^{\prime}$ dependence in the third argument. This leads to cancellation of the external potential term acting on particle 2 . $f^{(1)}$ changes by virtue of the influence of the external potential and through the interpar- ticle potential. The problem of computing $f_{2}$ is not evaded, however, by writing this equation.

\section{E. Boundary conditions}

In order to give the equation of motion in integral form, we need to specify the boundary condition. The in- 
coming density function for two free particles has to satisfy the homogeneous equation

$$
\left[\frac{\partial}{\partial t}+\underline{v}_{1} \cdot \nabla_{1}+\underline{v}_{2} \cdot \nabla_{2}\right) f_{\text {in }} \equiv \frac{D}{D t} f_{\text {in }}=0 .
$$

This equation is satisfied by any function of $R_{1}-V_{1} t$, $R_{2}-V_{2} t$. However, for two noninteracting incoming particles, we have more specifically as $t \rightarrow-\infty$,

$$
\begin{aligned}
f_{2} \rightarrow f_{2 \text { in }} & =f_{0}\left(\underline{p}_{1} \underline{R}_{1} t\right) f_{0}\left(\underline{p}_{2} \underline{R}_{2} t\right) \\
& =f_{0}\left(\underline{R}_{1}-\underline{v}_{1} t\right) f_{0}\left(\underline{R}_{2}-\underline{v}_{2} t\right) .
\end{aligned}
$$

Recall that each $f_{0}$ contains a reference position and a length parameter. In $(p, q, \omega)$ language, the incoming distribution is

$f_{2 \text { in }}=2 \pi f_{0}\left(\underline{p}_{1}, \underline{q}_{1}\right) f_{0}\left(\underline{p}_{2}, \underline{q}_{2}\right) \delta\left(\omega-\underline{q}_{1} \cdot \underline{v}_{1}-\underline{q}_{2} \cdot \underline{v}_{2}\right)$,

where, in terms of wave-packet coefficients

$$
f_{0}(\underline{p}, \underline{q}) \equiv C^{*}\left(\underline{p}-\frac{1}{2} \underline{q}\right) C\left(\underline{p}+\frac{1}{2} \underline{q}\right) \text {. }
$$

In the absence of external potentials, one generally extracts the $\mathrm{cm}$ motion and concentrates on the equivalent one-body problem. In this case, the change of variables $\left(x_{1}, x_{2}\right) \rightarrow(R, r)$ converts the homogeneous equation to

$$
\left[\frac{\partial}{\partial t}+V \cdot \nabla_{R}+v_{r} \cdot \nabla_{r}\right] f_{\text {in }}=0 \text {. }
$$

Writing $f_{\text {in }}$ in the special factorized form [Eq. (3.12)] then gives

$$
f_{\text {in }}=f_{0}(\underline{R}-\underline{V} t) f_{0}\left(\underline{r}-\underline{v}_{r} t\right) .
$$

Since the cm motion factors out [Eqs. (3.14) and (3.15)], the relative motion for the scattering problem is described by Eq. (2.44) with relative coordinates and $K$ computed from the potential $v$. In the case of Gaussian packets as in Eq. (2.16), Eqs. (3.29) and (3.30) are identical. Setting $R_{0}=0$ and taking the same width parameter $L$ for particles 1 and 2, we obtain by explicit calculation

$$
\left(\underline{p}_{1}-\underline{p}_{10}\right)^{2}+\left(\underline{p}_{2}-\underline{p}_{20}\right)^{2}=\frac{1}{2}\left(\underline{P}-\underline{P}_{0}\right)^{2}+2\left(\underline{p}-\underline{p}_{0}\right)^{2},
$$

where $P_{0}=p_{10}+p_{20}$ and $p_{0}=\frac{1}{2}\left(p_{10}-p_{20}\right)$.

Similarly for the space-time part,

$$
\left(\underline{R}_{1}-\underline{v}_{1} t\right)^{2}+\left(\underline{R}_{2}-\underline{v}_{2} t\right)^{2}=2(\underline{R}-\underline{V} t)^{2}+\frac{1}{2}(\underline{r}-\underline{v} t)^{2} .
$$

Hence we can write

$$
\begin{aligned}
& f_{0}\left(\underline{p}_{1} \underline{R}_{1} t\right) f_{0}\left(\underline{p}_{2} \underline{R}_{2} t\right) \\
& \quad=f_{0}\left[\underline{p}_{1}+\underline{p}_{2} \frac{\underline{R}_{1}+\underline{R}_{2}}{2}, t\right] f_{0}\left(\frac{\underline{p_{1}-\underline{p}_{2}}}{2}, \underline{R}_{1}-\underline{R}_{2}, t\right],
\end{aligned}
$$

where the length parameters occurring on the right-hand side are $L \rightarrow \frac{1}{2}$ in the first factor and $L \rightarrow 2 L$ in the second factor. It is a matter of convenience which approach to use, though the form (3.29) is more general and will generally be adhered to.

The integral form of the equation of motion following from Eqs. (3.18) and (3.26) is

$$
\begin{aligned}
f_{2}\left(\underline{p}_{1} \underline{R}_{2}, \underline{p}_{1} \underline{R}_{2} t\right)= & f_{2 \text { in }}\left(\underline{p}_{1} \underline{R}_{2}, \underline{p}_{1} \underline{R}_{2} t\right)+\int_{-\infty}^{t} d t^{\prime} \int d^{3} p_{1}^{\prime} \int d^{3} p_{2}^{\prime} K_{2}\left(\underline{p}_{1}^{\prime}-\underline{p}_{1}, \underline{p}_{2}^{\prime}-\underline{p}_{2}, \underline{R}_{1}^{\text {ret }}, \underline{R}_{2}^{\text {ret }}\right) f_{2}\left(\underline{p}_{1}^{\prime} \underline{R}_{1}^{\text {ret }} \underline{p}_{2}^{\prime} \underline{R}_{2}^{\text {ret }} t\right) \\
& +\int_{-\infty}^{t} d t^{\prime} \int d^{3} p_{1}^{\prime} K_{1}\left(\underline{p}_{1}^{\prime}-\underline{p}_{1}, \underline{R}_{1}^{\text {ret }}, t^{\prime}\right) f_{2}\left(\underline{p}_{1}^{\prime} \underline{R}_{1}^{\text {ret }}, \underline{p}_{2} \underline{R}_{2}^{\text {ret }} t^{\prime}\right) \\
& +\int_{-\infty}^{t} d t^{\prime} \int d^{3} p_{2}^{\prime} K_{1}\left(\underline{p}_{2}^{\prime}-\underline{p}_{2}, \underline{R}_{2}^{\text {ret }}, t^{\prime}\right) f_{2}\left(\underline{p}_{1} \underline{R}_{1}^{\text {ret }}, \underline{p}_{2}^{\prime} \underline{R}_{2}^{\text {ret }} t^{\prime}\right) .
\end{aligned}
$$

The retarded variables are defined as in the one-particle case [Eq. (2.44)]

$$
\begin{aligned}
& R_{1}^{\text {ret }}=R_{1}-v_{p_{1}}\left(t-t^{\prime}\right), \\
& R_{2}^{\text {ret }}=R_{2}-v_{p_{2}}\left(t-t^{\prime}\right) .
\end{aligned}
$$

The $K_{2}$ term can be simplified by using Eq. (3.10).

If the two-particle system exhibits bound states, then Eq. (3.34) can induce "capture" if the external potentials can supply the requisite energy and momentum. Such problems are handled as in Sec. II.H. As tacitly understood in the one-body problem, there is no translation invariance, and $V_{\text {ex }}$ must be referred to a particular coordinate system.

Note that Eq. (3.34) contains as special cases two distinct one-body problems. As already seen, this equation reduces to the one-body problem for relative motion when the external potentials are zero. We can also arrange the incident packets so that particle 1 scatters off $V_{1}$ but misses particle 2 completely. In the general case, when the two particles scatter off each other in the presence of external potentials, the problem is much more complex. Although this is the prototype of the important practical problem of scattering in a medium, we shall not pursue it here.

\section{THE N-BODY PROBLEM: HIERARCHIES AND THE BOLTZMANN-VLASOV EQUATION}

\section{A. Definitions and equation of motion}

We extend the previous development to an $N$-body system with Hamiltonian

$$
H=\sum_{i=1}^{N} \frac{p_{i}^{2}}{2 m}+\sum_{i<j} v\left(x_{i}-x_{j}\right)+\sum_{i} V_{i}\left(x_{i}, t\right) .
$$

For simplicity we assume that the particles are formally distinguishable but have identical properties. From the $N$-particle wave function $\psi\left(x_{1} \cdots x_{N}, t\right)$ we form the distribution function 


$$
\begin{aligned}
f_{N}\left(p_{1} R_{1}, p_{2} R_{2} \cdots p_{N} R_{N} t\right) & \\
=\int_{j=1}^{N} \prod & \frac{d^{3} r_{j}}{(2 \pi)^{3}} e^{-p_{j} \cdot r_{j}} \\
& \times \psi^{*}\left(R_{1}-\frac{1}{2} r_{1}, \ldots, R_{N}-\frac{1}{2} r_{N}, t\right) \\
& \times \psi\left(R_{1}+\frac{1}{2} r_{1}, \ldots, R_{N} \frac{1}{2} r_{N}, t\right) .
\end{aligned}
$$

As in the two-body problem, it is useful to define an array of coordinates, mainly dealing with pairs of particles:

$$
\begin{aligned}
& x_{i}=R_{i}-\frac{1}{2} r_{i}, \quad x_{i}^{\prime}=R_{i}+\frac{1}{2} r_{i}, \\
& R_{i j}=\frac{1}{2}\left(R_{i}+R_{j}\right), \quad r_{i j}=R_{i}-R_{j}, \\
& \rho_{i j}=r_{i}-r_{j}, \quad \widetilde{R}_{i j}=\frac{1}{2}\left(r_{i}+r_{j}\right), \\
& P=\sum_{i} p_{i}, \quad R=\sum_{i} R_{i} / N .
\end{aligned}
$$

$f_{N}$ can also be expressed in terms of the momentum basis expansion coefficients as in Eq. (3.4). An equation of motion for $f_{N}$ is derived from the Schrödinger equation as before $\left(v_{i}\right.$ is $p_{i} / m$ and $\nabla_{i}$ is $\left.\partial / \partial \mathbf{R}_{i}\right)$ :

$$
\begin{aligned}
\left(\frac{\partial}{\partial t}+\sum v_{i} \cdot \nabla_{i}\right] f_{N}\left(p_{1} R_{1} \cdots p_{N} R_{N} t\right)= & \int \prod_{i} d^{3} p_{i}^{\prime} K\left(p_{1}^{\prime}-p_{1} \cdots p_{N}^{\prime}-p_{N}, R_{1} \cdots R_{N}\right) f_{N}\left(p_{1}^{\prime} R_{1} p_{2}^{\prime} R_{2} \cdots p_{N}^{\prime} R_{N} t\right) \\
& +\sum_{j=1}^{N} \int d^{3} p_{j}^{\prime} K_{1}\left(p_{j}^{\prime}-p_{j}, R_{j}, t\right) f_{N}\left(p_{1} \cdots p_{j}^{\prime} R_{j} \cdots t\right)
\end{aligned}
$$

In the absence of external potentials, one can factor out the $\mathrm{cm}$ motion in analogy to Eq. (3.11). In the following we drop the external potential terms and concentrate on the two-body potential terms. The kernel $K$,

$$
K\left\{p_{i}^{\prime}-p_{i}, R_{i}\right\} \equiv \frac{1}{i} \int \prod_{j}\left[\frac{d^{3} r_{j}}{(2 \pi)^{3}} e^{i\left(p_{j}^{\prime}-p_{j}\right) \cdot r_{j}}\right] \sum_{i<j}\left[v\left(x_{i}^{\prime}-x_{j}^{\prime}\right)-v\left(x_{i}-x_{j}\right)\right],
$$

actually changes only two momenta at a time, since $v$ is a pair potential. Explicitly

$$
\begin{aligned}
i K\left\{p_{i}^{\prime}-p_{i}, R_{i}\right\}=\sum_{i<j} \int \frac{d^{3} q_{i} d^{3} q_{j}}{(2 \pi)^{3}} v\left(q_{i}, q_{j}\right) e^{i q_{i} \cdot R_{i}+i q_{j} \cdot R_{j}} \\
\quad \times\left[\begin{array}{l}
\delta\left(p_{1}^{\prime}-p_{1}\right) \cdots \delta\left(p_{i}^{\prime}-p_{i}+\frac{1}{2} q_{i}\right) \cdots \delta\left(p_{j}^{\prime}-p_{j}+\frac{1}{2} q_{j}\right) \cdots \delta\left(p_{N}^{\prime}-p_{N}\right) \\
\left.\quad-\delta\left(p_{1}^{\prime}-p_{1}\right) \cdots \delta\left(p_{i}^{\prime}-p_{i}-\frac{1}{2} q_{i}\right) \cdots \delta\left(p_{j}^{\prime}-p_{j}-\frac{1}{2} q_{j}\right) \cdots \delta\left(p_{N}^{\prime}-p_{N}\right)\right] .
\end{array}\right.
\end{aligned}
$$

Since $v$ depends on coordinate differences, we have further

$$
v\left(q_{i}, q_{j}\right)=(2 \pi)^{3} \delta\left(q_{i}+q_{j}\right) v\left(\frac{q_{i}-q_{j}}{2}\right)
$$

Again the resultant equation of motion can be written in several forms. In $p, q, \omega$ variables we have (neglecting external potentials)

$$
\begin{aligned}
& {\left[\omega-\sum_{i} q_{i} v_{i}\right] f_{N}\left(p_{1} q_{1} \cdots p_{N} q_{N} \omega\right)} \\
& =\sum_{i<j} \int \frac{d^{3} q_{i}^{\prime}}{(2 \pi)^{3}} v\left(q_{i}^{\prime}\right)\left[f_{N}\left(p_{1} q_{1} \cdots p_{i}-\frac{1}{2} q_{i}^{\prime}, q_{i}-q_{i}^{\prime}, \ldots, p_{j}+\frac{1}{2} q_{i}^{\prime}, q_{j}+q_{i}^{\prime} \cdots \omega\right)\right. \\
& \left.\quad-f_{N}\left(p_{1} q_{1} \cdots p_{i}+\frac{1}{2} q_{i}^{\prime}, q_{i}-q_{i}^{\prime}, \ldots, p_{j}-\frac{1}{2} q_{i}^{\prime}, q_{j}+q_{i}^{\prime} \cdots \omega\right)\right],
\end{aligned}
$$

using Eq. (4.7) to eliminate $q_{j}^{\prime}$.

\section{B. Coupled hierarchy equations for reduced distribution functions}

As in statistical mechanics, one can usefully define reduced distribution functions by integrating out various degrees of freedom. These reduced distributions then obey a coupled hierarchy of equations: 


$$
\begin{aligned}
& f_{N}^{(1)}\left(p_{1} R_{1} t\right) \equiv \int \prod_{i=2}^{N} d^{3} p_{j} d^{3} R_{j} f_{N}\left(p_{1} R_{1} \cdots p_{N} R_{N} t\right) \\
& f_{N}^{(2)}\left(p_{1} R_{1} p_{2} R_{2} t\right) \equiv \int \prod_{i=3}^{N} d^{3} p_{j} d^{3} R_{j} f_{N}\left(p_{1} R_{1} \cdots p_{N} R_{N} t\right) \\
& f_{N}^{(n)}\left(p_{1} R_{1} \ldots p_{n} R_{n} t\right) \equiv \int \prod_{i=n+1}^{N} d^{3} p_{j} d^{3} R_{j} f_{N}\left(p_{1} R_{1} \cdots p_{N} R_{N} t\right)
\end{aligned}
$$

Note that in $p, q, \omega$ language the space integrations amount to setting $q=0$,

$$
f_{N}^{(n)}\left(p_{1} q_{1} \ldots p_{n} q_{n} \omega\right)=\int \prod_{j=n+1}^{N} d^{3} p_{j} f_{N}\left(p_{1} q_{1} \cdots p_{n} q_{n} p_{n+1} \mathbf{0} \cdots p_{N} \mathbf{0}, \omega\right) .
$$

Applying this rule to the equation of motion to get $f_{N}^{(1)}$, we find

$$
\begin{aligned}
\left(\omega-\underline{q}_{1} \cdot \underline{v}_{1}\right) f_{N}^{(1)}\left(p_{1} q_{1} \omega\right)=\sum_{i<j} \int & d^{3} p_{2} \cdots d^{3} p_{N} \frac{d^{3} q_{i}^{\prime}}{(2 \pi)^{3}} v\left(q_{i}^{\prime}\right) \\
& \times\left[f_{N}\left(p_{1}, q_{1}, p_{2} 0, \ldots, p_{i}-\frac{1}{2} q_{i}^{\prime}, q_{i}-q_{i}^{\prime} \cdots p_{j}+\frac{1}{2} q_{j}^{\prime}, q_{j}+q_{j}^{\prime}, \ldots, \omega\right)-\cdots\right] .
\end{aligned}
$$

Note that $q_{j}$ is always zero, since $j>i \geq+1$. Now consider separately the $i=1$ and $i>1$ terms on the right-hand side. For $i=1$ we find

$$
\begin{gathered}
\sum_{j>1} \int \frac{d^{3} q_{1}^{\prime}}{(2 \pi)^{3}} v\left(q_{1}^{\prime}\right) \int d^{3} p_{2} \cdots d^{3} p_{N}\left[f_{N}\left(p_{1}-\frac{1}{2} q_{1}^{\prime}, q_{1}-q_{1}^{\prime} p_{2} 0, \ldots, p_{j}+\frac{1}{2} q_{1}^{\prime}, q_{1}^{\prime}, \ldots, p_{N} 0 \omega\right)\right. \\
\left.-f_{N}\left(p_{1}+\frac{1}{2} q_{1}^{\prime}, q_{1}-q_{1}^{\prime}, p_{2} 0, \ldots, p_{j}-\frac{1}{2} q_{1}^{\prime}, q_{1}^{\prime} \ldots p_{N} 0 \omega\right)\right] \\
=\sum_{j>1} \int d^{3} p_{j} \int \frac{d^{3} q_{1}^{\prime}}{(2 \pi)^{3}} v\left(q_{1}^{\prime}\right)\left[f_{N}^{(2)}\left(p_{1}-\frac{1}{2} q_{1}^{\prime}, q_{1}-q_{1}^{\prime}, p_{j}+\frac{1}{2} q_{1}^{\prime}, q_{1}^{\prime}, \ldots, p_{N} \omega\right)\right. \\
\left.-f_{N}^{(2)}\left(p_{1}+\frac{1}{2} q_{1}^{\prime}, q_{1}-q_{1}^{\prime}, p_{j}-\frac{1}{2} q_{1}^{\prime}, q_{1}^{\prime}, \ldots, p_{N} \omega\right)\right],
\end{gathered}
$$

recalling the definition of $f^{(2)}$. This equation has $N-1$ equal contributions to the sum.

All terms having $i \geq 2$ cancel, as shown by the following expression:

$$
\begin{aligned}
\sum_{j>2} \int \frac{d^{3} q_{2}^{\prime}}{(2 \pi)^{3}} \int v\left(q_{2}^{\prime}\right) d^{3} p_{2} \cdots d^{3} p_{N}[ & f_{N}\left(p_{1} q_{1}, p_{2}-\frac{1}{2} q_{2}^{\prime},-q_{2}^{\prime}, \ldots, p_{j}+\frac{1}{2} q_{2}^{\prime}, q_{2}^{\prime}, \ldots, p_{N} 0 \omega\right) \\
& \left.-f_{N}\left(p_{1} q_{1}, p_{2}+\frac{1}{2} q_{2}^{\prime}-q_{2}^{\prime} \cdots p_{j}-\frac{1}{2} q_{2}^{\prime}, q_{2}^{\prime} \cdots p_{N} 0 \omega\right)\right]
\end{aligned}
$$

Now the apparent $\pm q_{2}^{\prime} / 2$ dependence of the $p_{2}$ and $p_{j}$ arguments can be removed by shifting integration variables and canceling. We are left with

$$
\begin{array}{r}
\left(\omega-\underline{q}_{1} \cdot \underline{v}_{1}\right) f_{N}^{(1)}\left(p_{1} q_{1} \omega\right)=(N-1) \int \frac{d^{3} q_{1}^{\prime}}{(2 \pi)^{3}} v\left(q_{1}^{\prime}\right) \int d^{3} p_{2}\left[f_{N}^{(2)}\left(p_{1}-\frac{1}{2} q_{1}^{\prime}, q_{1}-q_{1}^{\prime}, p_{2}+\frac{1}{2} q_{1}^{\prime} q_{1}^{\prime}, \omega\right)\right. \\
\left.-f_{N}^{(2)}\left(p_{1}+\frac{1}{2} q_{1}^{\prime}, q_{1}-q_{1}^{\prime}, p_{2}-\frac{1}{2} q_{1}^{\prime}, q_{1}^{\prime} \omega\right)\right]
\end{array}
$$

For $N=2$ this reduces to the equation formed before [Eq. (3.27)] in the two-body problem. Note that, if desired, we may remove the $q_{1}^{\prime}$ variable accompanying the $p_{2}$ integration.

For $N=2$ we encounter a new feature which extends to all higher-order distribution functions. Proceeding as before, we find

$$
\begin{aligned}
& \left(\omega-q_{1} \cdot v_{1}-q_{2} \cdot v_{2}\right) f_{N}^{(2)}\left(p_{1} q_{1} p_{2} q_{2} \omega\right) \\
& =\sum_{i<j} \int \frac{d^{3} q_{1}^{\prime}}{(2 \pi)^{3}} v\left(q_{1}^{\prime}\right) \int d^{3} p_{3} \cdots d^{3} p_{N}\left[f_{N}\left(p_{1} q_{1} p_{2} q_{2} p_{3} 0, \ldots, p_{i}-\frac{1}{2} q_{i}^{\prime}, q_{i}-q_{i}^{\prime}, \ldots, p_{j}+\frac{1}{2} q_{i}^{\prime}, q_{j}+q_{i}^{\prime}, \ldots, p_{N} 0 \omega\right)\right. \\
& \left.\quad-f_{N}\left(p_{1} q_{1} p_{2} q_{2} p_{3} 0, \ldots, p_{i}+\frac{1}{2} q_{i}^{\prime}, q_{i}-q_{i}^{\prime}, \ldots, p_{j}-\frac{1}{2} q_{i}^{\prime}, q_{j}+q_{i}^{\prime}, \ldots, p_{N} 0 \omega\right)\right] .
\end{aligned}
$$


First note that if $i \geq 3$, we can again shift integration variables so that the apparent $f^{(4)}$ terms cancel, etc. The $i=1,2$ contributions remain to be studied.

For $i=1, j=2$, we find

$$
\int \frac{d^{3} q_{1}^{\prime}}{(2 \pi)^{3}} v\left(q_{1}^{\prime}\right)\left[f_{N}^{(2)}\left(p_{1}-\frac{1}{2} q_{1}^{\prime}, q_{1}-q_{1}^{\prime}, p_{2}+\frac{1}{2} q_{1}^{\prime}, q_{2}+q_{1}^{\prime}, \omega\right)-f_{N}^{(2)}\left(p_{1}+\frac{1}{2} q_{1}^{\prime}, q_{1}-q_{1}^{\prime}, p_{2}-\frac{1}{2} q_{1}^{\prime}, q_{2}+q_{1}^{\prime}, \omega\right)\right] .
$$

For $i=1, j \geq 3$, we find

$$
\sum_{j \geq 3} \int d^{3} p_{j} \int \frac{d^{3} q_{1}^{\prime}}{(2 \pi)^{3}} v\left(q_{1}^{\prime}\right)\left[f_{3}\left(p_{1}-\frac{1}{2} q_{1}^{\prime}, q_{1}-q_{1}^{\prime}, p_{2} q_{2}, p_{j}+\frac{1}{2} q_{1}^{\prime}, q_{1}^{\prime} \omega\right)-f_{3}\left(p_{1}+\frac{1}{2} q_{1}^{\prime}, q_{1}-q_{1}^{\prime}, p_{2} q_{2} p_{j}-\frac{1}{2} q_{1}^{\prime}, q_{1}^{\prime}, \omega\right)\right],
$$

which has $N-2$ identical terms. For $i=2, j \geq 3$, the result is the same except that particle 1 stands by while particles 2 and 3 scatter.

Combining these results gives the second equation of the hierarchy,

$$
\begin{aligned}
& \left(\omega-\underline{v}_{1} \underline{v}_{1}-q_{2} \cdot v_{2}\right) f_{N}^{(2)}\left(p_{1}, q_{1}, p_{2} q_{2} \omega\right) \\
& =(N-1) \int \frac{d^{3} q_{1}^{\prime}}{(2 \pi)^{3}} v\left(q_{1}^{\prime}\right)\left[f_{N}^{(2)}\left(p_{1}-\frac{1}{2} q_{1}^{\prime}, q_{1}-q_{1}^{\prime}, p_{2}+\frac{1}{2} q_{1}^{\prime}, q_{2}+q_{1}^{\prime} \omega\right)-f_{N}^{(2)}\left(p_{1}+\frac{1}{2} q_{1}^{\prime}, q_{1}-q_{1}^{\prime}, p_{2}-\frac{1}{2} q_{1}^{\prime}, q_{2}+q_{1}^{\prime}, \omega\right)\right] \\
& \quad+(N-2) \int d^{3} p^{\prime} \int \frac{d^{3} q_{1}^{\prime}}{(2 \pi)^{3}} v\left(q_{1}^{\prime}\right)\left[f_{N}^{(3)}\left(p_{1}-\frac{1}{2} q_{1}^{\prime}, q_{1}-q_{1}^{\prime}, p_{2} q_{2}, p^{\prime}+\frac{1}{2} q_{1}^{\prime}, q_{1}^{\prime} \omega\right)\right. \\
& \left.\quad-f_{N}^{(3)}\left(p_{1}+\frac{1}{2} q_{1}^{\prime}, q_{1}-q_{1}^{\prime}, p_{2} q_{2}, p^{\prime}-\frac{1}{2} q_{1}^{\prime}, q_{1}^{\prime} \omega\right)\right] \\
& +(N-2) \int d^{3} p^{\prime} \int \frac{d^{3} q_{2}^{\prime}}{(2 \pi)^{3}} v\left(q_{2}^{\prime}\right)\left[f_{N}^{(3)}\left(p_{1} q_{1}, p_{2}-\frac{1}{2} q_{2}^{\prime}, q_{2}-q_{2}, p^{\prime}+\frac{1}{2} q_{2}^{\prime}, q_{2}^{\prime} \omega\right)\right. \\
& \left.\quad-f_{N}^{(3)}\left(p_{1} q_{, 1} p_{2}+\frac{1}{2} q_{2}^{\prime}, q_{2}-q_{2}^{\prime}, p^{\prime}-\frac{1}{2} q_{2}^{\prime}, q_{2}^{\prime} \omega\right)\right] .
\end{aligned}
$$

For $N=2$ the surviving terms are just as found before in Sec. II.

The physical significance of this result is quite clear: $f_{N}^{(2)}$ changes by (a) collisions between the selected particles 1 and 2 or by (b) collisions between 1 and $n \geq 3$ or by (c) collisions between 2 and $n>3$ (Fig. 7).

The $n$ th-order equation is given by

$$
\begin{aligned}
&\left(\omega-\sum_{j=1}^{n} q_{j} \cdot v_{j}\right] f_{N}^{(n)}\left(p_{1} q_{1} \cdots p_{n} q_{n} \omega\right) \\
&=(N-n+1) \int \frac{d^{3} q_{1}^{\prime}}{(2 \pi)^{3}} v\left(q_{1}^{\prime}\right)\left[f_{N}^{(n)}\left(p_{1}-\frac{1}{2} q_{1}^{\prime}, q_{1}-q_{1}^{\prime}, p_{2}+\frac{1}{2} q_{1}^{\prime}, q_{1}^{\prime}, p_{3} q_{3} \cdots p_{n} q_{n} \omega\right)\right. \\
&\left.\quad-f_{N}^{(n)}\left(p_{1}+\frac{1}{2} q_{1}^{\prime}, q_{1}-q_{1}^{\prime}, p_{2}-\frac{1}{2} q_{1}^{\prime}, q_{1}^{\prime} p_{3} q_{3} \cdots p_{n} q_{n} \omega\right)\right]
\end{aligned}
$$

$+\sum$ (permutations on all pairs)

$$
\begin{aligned}
+(N-n) \int d^{3} p^{\prime} \int \frac{d^{3} q_{1}^{\prime}}{(2 \pi)^{3}} v\left(q_{1}^{\prime}\right) \\
\quad \times\left[f_{N}^{(n+1)}\left(p_{1}-\frac{1}{2} q_{1}^{\prime}, q_{1}-q_{1}^{\prime}, p_{2} q_{2} \cdots p_{n} q_{n}, p^{\prime}+\frac{1}{2} q_{1}^{\prime}, q_{1}^{\prime} \omega\right)\right. \\
\left.\quad-f_{N}^{(n+1)}\left(p_{1}+\frac{1}{2} q_{1}^{\prime}, q_{1}-q_{1}^{\prime}, p_{2} q_{2} \cdots p_{n} q_{n}, p^{\prime}-\frac{1}{2} q_{1}^{\prime}, q_{1}^{\prime}, \omega\right)\right]
\end{aligned}
$$

$+\sum$ (permutations of 1 with $2,3, \ldots n$ ).

A simple figure (Fig. 8) illustrates the physical content of the hierarchy. We select $n$ particles and ask how $f^{(n)}\left(p_{1} R_{1} \cdots p_{n} R_{n} t\right)$ changes with time. This change is due to two-body collisions of particles among the first $n$, or collisions between one of the $n$ and one of the $N-n$. Collisions among the $N-n$ "other" particles do not directly affect $f^{(n)}$.

The development of this and the preceding section is 

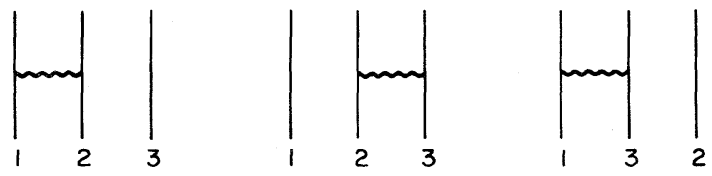

FIG. 7. Three kinds of contributions to the equation of motion for $f_{2}$ [see Eq. (4.18)]. In (a) the two chosen particles scatter, while in (b) and (c) one of the chosen particles scatters off a particle in the medium while the other chosen particle is unaffected.

completely general insofar as it is only a restatement of the $N$-body equations of motion in phase-space language. However, as soon as we postulate boundary conditions or make an approximation to the hierarchy (typically factorization, leading to truncation), careful attention has to be given to physical requirements. For example, in a collision of composite systems, the particles in a bound state are always correlated initially, and one cannot factorize a distribution function in the variables of the bound state.

In the scattering of composites having $N_{1}, N_{2}$ particles ( $N_{i}$ assumed large), there can be a region of space-time in which the dynamics can be described by kinetic equations of the traditional type. As a preliminary to the discussion of scattering of composites, we review in our formalism some traditional simplifications possible for large (not necessarily uniform) systems.

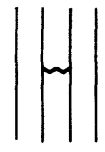

n

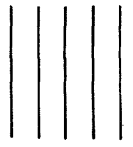

$N-n$

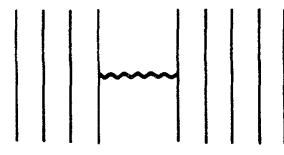

$N-n$

FIG. 8. Contributions to the $n$-particle reduced distribution function. These comprise a simple generalization of Fig. 7.

\section{Truncation schemes \\ and the Boltzmann-Vlasov equation}

Although there is no way to legitimately avoid the $\mathrm{N}$ body problem, many physical quantities depend on highly averaged objects like the one- and the two-body distribution functions. In many cases, physically motivated (but mathematically dubious) approximations, such as truncation, neglect of correlations, etc., lead to good results with highly intuitive content. In traditional subjects these approximations are associated with the names of Hartree, Vlasov, and Boltzmann. The validity of these approximation schemes in complicated scattering and reaction processes remains to be studied. As a preliminary, we show how the Vlasov and Boltzmann equations can be extracted from our formalism.

The equation of motion for the one-particle distribution functions including a time-dependent external potential is

$$
\begin{aligned}
\left\lfloor i \frac{\partial}{\partial t}-q_{1} \cdot v_{1}\right] f_{N}^{(1)}\left(p_{1} q_{1} t\right) \\
=(N-1) \int \frac{d^{3} q_{1}^{\prime}}{(2 \pi)^{3}} v\left(q_{1}^{\prime}\right) \int d^{3} p_{2}\left[f_{N}^{(2)}\left(p_{1}-\frac{1}{2} q_{1}^{\prime}, q_{1}-q_{1}^{\prime}, p_{2} q_{1}^{\prime} t\right)-f_{N}^{(2)}\left(p_{1}+\frac{1}{2} q_{1}^{\prime}, q_{1}-q_{1}^{\prime}, p_{2}, q_{1}^{\prime}, t\right)\right] \\
\quad+\int \frac{d^{3} q_{1}^{\prime}}{(2 \pi)^{3}} V\left(q_{1}^{\prime}, t\right)\left[f_{N}^{(1)}\left(p_{1}-\frac{1}{2} q_{1}^{\prime}, q_{1}-q_{1}^{\prime}, t\right)-f_{N}^{(1)}\left(p_{1}+\frac{1}{2} q_{1}^{\prime}, q_{1}-q_{1}^{\prime}, t\right)\right] .
\end{aligned}
$$

Assuming that particles 1 and 2 are uncorrelated, i.e.,

$$
f^{(2)}\left(p_{1} R_{1}, p_{2} R_{2} t\right)=f^{(1)}\left(p_{1} R_{1} t\right) f^{(1)}\left(p_{2} R_{2} t\right),
$$

we obtain a nonlinear equation for $f^{(1)}$,

$$
\left\{i \frac{\partial}{\partial t}-q_{1} \cdot v_{1}\right\rfloor f^{(1)}(p, q, t)=\int \frac{d^{3} q_{1}^{\prime}}{(2 \pi)^{3}} V_{\mathrm{eff}}\left(q^{\prime}, t\right)\left[f^{(1)}\left(p_{1}-\frac{1}{2} q_{1}^{\prime}, q_{1}-q_{1}^{\prime}, t\right)-f^{(1)}\left(p_{1}+\frac{1}{2} q_{1}^{\prime}, q_{1}-q_{1}^{\prime}, t\right)\right] .
$$

This is the usual mean-field theory expressed in phasespace language, where the effective potential energy of a particle is

$$
\begin{aligned}
V_{\text {eff }}\left(q_{1}^{\prime}, t\right)= & V\left(q_{1}^{\prime}, t\right) \\
& +(N-1) v\left(q_{1}^{\prime}\right) \int d^{3} p_{2} f^{(1)}\left(p_{2}, q_{1}^{\prime}, t\right) .
\end{aligned}
$$

Equation (4.22) is the time-dependent Hartree-Vlasov equation in which the distribution $f^{(1)}$ may be imagined to be found self-consistently. When linearized, it gives a random-phase approximation which exhibits under suitable circumstances collective modes such as plasmons and zero sound in uniform systems.

For a system of electrons (neutralized by a constant positive background) the resultant equation is the Vlasov equation. We review the case of small oscillations about a spatially uniform Maxwellian distribution normalized to one particle per unit volume:

$$
\begin{aligned}
& f^{(1)}(p, q, t)=f_{0}(p)(2 \pi)^{3} \delta(q)+f^{\prime}(p, q, t) \\
& f_{0}(p, R, t)=\frac{\exp \left(-p^{2} / 2 m k T\right)}{(2 \pi m k T)^{3 / 2}} .
\end{aligned}
$$

If we assume $f^{\prime}$ to be of order $V\left(q_{1}^{\prime} t\right)$, linearization gives 


$$
\begin{aligned}
f^{\prime}(p, q, \omega)= & X(p, q, \omega) \\
& \times\left[V(q, \omega)+(N-1) v(q) \int d^{3} p^{\prime} f^{\prime}\left(p^{\prime}, q, \omega\right)\right], \\
X(p, q, \omega)= & \frac{f_{0}\left(p-\frac{1}{2} q\right)-f_{0}\left(p+\frac{1}{2} q\right)}{\omega+i \varepsilon-\underline{q} \cdot \underline{v}_{p}} .
\end{aligned}
$$

The solution is

$$
f^{\prime}(p, q, \omega)=\frac{V(q, \omega) X(p, q, \omega)}{1-(N-1) v(q) \int d^{3} p X(p, q, \omega)} .
$$

If collective modes exist for frequency $\omega(q)$, then $f^{\prime}$ can be finite for arbitrarily small $V(q, \omega)$. The eigenvalue equation is

$$
1=(N-1) v(q) P \int d^{3} p \frac{f_{0}\left(p-\frac{1}{2} q\right)-f_{0}\left(p+\frac{1}{2} q\right)}{\omega-\underline{q} \cdot \underline{v}_{p}},
$$

where $P$ stands for the principle value. In the Coulomb gas, we can find the plasmon root for $q \rightarrow 0$ by expanding the integrand,

$$
\begin{aligned}
& 1=(N-1) v(q) \int d^{3} p\left[-\underline{q} \cdot \nabla_{p} f_{0}(p)\right. \\
& \left.\times\left[\frac{1}{\omega}+\frac{q \cdot p / m}{\omega^{2}}+\cdots\right]\right], \\
& 1=(N-1) \frac{v(q) q^{2}}{m \omega^{2}} \int d^{3} p f_{0}(p), \\
& \omega_{\mathrm{pl}}^{2}=\frac{4 \pi N e^{2}}{V m} \text {. }
\end{aligned}
$$

A more detailed discussion is given by Brout and Carruthers (1968). Here we have converted $N$ to a density $N / V$ to conform to our normalization.

All these results are well known. To proceed, we want to place the above in a more ambitious context, that of improving the above approximation to obtain the Boltzmann collision terms. Suppose we integrate formally the equation for $f^{(2)}$ to include collisions between 1 and 2 , but ignore correlations in $f^{(3)}$. This approximation leads to the usual Boltzmann equation with the cross section evaluated in lowest Born approximation, but with a particular factorization approximation for $f^{(3)}$.

For our approximate $f^{(2)}$, we make the ansatz

$$
\begin{aligned}
\widetilde{f}^{(2)}\left(p_{1} q_{1} p_{2} q_{2} t\right)= & \widetilde{f}^{(1)}\left(p_{1} q_{1} t\right) \widetilde{f}^{(1)}\left(p_{2} q_{2} t\right) \\
& +f_{-\infty}^{t} d t^{\prime} e^{-i\left(q_{1} v_{1}+q_{2}, v_{2}\right)\left(t-t^{\prime}\right)} \int \frac{d^{3} q_{1}^{\prime}}{(2 \pi)^{3}} v\left(q_{1}^{\prime}\right)\left[\widetilde{f}^{(2)}\left(p_{1}-\frac{1}{2} q_{1}^{\prime}, q_{1}-q_{1}^{\prime}, p_{2}+\frac{1}{2} q_{1}^{\prime}, q_{1}^{\prime} t^{\prime}\right)\right. \\
& \left.-\widetilde{f}^{(2)}\left(p_{1}+\frac{1}{2} q_{1}^{\prime} q_{1}-q_{1}^{\prime}, p_{2}-\frac{1}{2} q_{1}^{\prime} q_{1}^{\prime} t^{\prime}\right)\right] .
\end{aligned}
$$

$\widetilde{f}_{1}$ is then the solution to Eq. (4.20) with $f_{2} \rightarrow \widetilde{f}_{2}$. Formally this is the two-body scattering problem in the absence of the rest of the system, without, however, the $\mathrm{cm}$ momentum constraint, i.e., $p_{1}+p_{2}$ is not some fixed constant. To see in what sense this is an approximate solution, we apply $D / D t$ to this expression using the equation of motion [Eq. (4.20)] for $f^{(1)}$,

$$
\begin{aligned}
&\left.\int i \frac{\partial}{\partial t}-q_{1} \cdot v_{1}-q_{2} \cdot v_{2}\right) \widetilde{f}^{(2)}=\int \frac{d^{3} q_{1}^{\prime}}{(2 \pi)^{3}} v\left(q_{1}^{\prime}\right)\left[\widetilde{f}^{(2)}\left(p_{1}-\frac{1}{2} q_{1}^{\prime}, q_{1}-q_{1}^{\prime}, p_{2}+\frac{1}{2} q_{1}, q_{1}^{\prime} t\right)\right. \\
&\left.-\widetilde{f}^{(2)}\left(p_{1}+\frac{1}{2} q_{1}^{\prime}, q_{1}-q_{1}^{\prime}, p_{2}-\frac{1}{2} q_{1}^{\prime}, q_{1}^{\prime} t\right)\right] \\
&+(N-1)\left(\int \frac { d ^ { 3 } q _ { 1 } ^ { \prime } } { ( 2 \pi ) ^ { 3 } } v ( q _ { 1 } ^ { \prime } ) \int d ^ { 3 } p _ { 2 } ^ { \prime } \left[\widetilde{f}^{(2)}\left(p_{1}-\frac{1}{2} q_{1}^{\prime}, q_{1}-q_{1}^{\prime}, p_{2}^{\prime}+\frac{1}{2} q_{1}^{\prime}, q_{1}^{\prime} t\right)\right.\right. \\
&\left.-\widetilde{f}^{(2)}\left(p_{1}+\frac{1}{2} q_{1}^{\prime}, q_{1}-q_{1}^{\prime}, p_{2}-\frac{1}{2} q_{1}^{\prime}, q_{1}^{\prime}, t\right)\right] \\
&\left.\times f^{(1)}\left(p_{2} q_{2} t\right)+(1 \leftrightarrow 2)\right) .
\end{aligned}
$$

Comparing with the exact equation of motion for $f^{(2)}$, we see that the approximation $f^{(2)}=\widetilde{f}^{(2)}$ corresponds to the following factorization:

$$
f^{(3)}\left(p_{1}-\frac{1}{2} q_{1}^{\prime}, q_{1}-q_{1}^{\prime}, p_{2} q_{2}, p^{\prime}+\frac{1}{2} q_{1}^{\prime}, q_{1}^{\prime} t\right)=f^{(2)}\left(p_{1}-\frac{1}{2} q_{1}^{\prime} q_{1}-q_{1}^{\prime}, p^{\prime}+\frac{1}{2} q_{1}^{\prime}, q_{1}^{\prime} t\right) f^{(1)}\left(p_{2}, q_{2}, t\right),
$$

etc., i.e., factorization of the one-particle distribution for the unscattered particle, and secondly $N-1 \approx N-2$, requiring $N \gg 1$ for validity. 
Clearly, a similar procedure can be adopted in any order: factor the $\boldsymbol{n}+1$-particle distribution suitably and solve for $f^{(n)}$ each $n$ in turn. "Suitable" factorization is a subject for further study, and the question of convergence is completely open.

For the moment, our ambition is only to show how both the Boltzmann and the Vlasov contributions to the oneparticle transport equation follow systematically from our ansatz for $f^{(2)}$. Iterating once by substituting $f^{(2)}$ into the right-hand side of Eq. (4.20), we find

$$
\begin{aligned}
& {\left[i \frac{\partial}{\partial t}-q v\right] f^{(1)}(p, q, t)-\int \frac{d^{3} q^{\prime}}{(2 \pi)^{3}} V_{\mathrm{eff}}\left(q^{\prime}, t\right)\left[f^{(1)}\left(p-\frac{1}{2} q^{\prime}, q-q^{\prime}, t\right)-f^{(1)}\left(p+\frac{1}{2} q^{\prime}, q-q^{\prime}, t\right)\right]} \\
& =(N-1) \int \frac{d^{3} q^{\prime}}{(2 \pi)^{3}} \int \frac{d^{3} q^{\prime \prime}}{(2 \pi)^{3}} v\left(q^{\prime}\right) v\left(q^{\prime \prime}\right) \\
& \times \int d^{3} p^{\prime}\left\{\int_{-\infty}^{t} d t^{\prime} \exp \left[-i\left[\left(q-q^{\prime}\right) \frac{p-\frac{1}{2} q^{\prime}}{m}+q^{\prime}\left(p^{\prime}+\frac{1}{2} q^{\prime}\right)\right]\left(t-t^{\prime}\right)\right]\right. \\
& \times\left[f ^ { ( 2 ) } \left(p-\frac{1}{2} q^{\prime}-\frac{1}{2} q^{\prime \prime}, q-q^{\prime}-q^{\prime \prime}, p^{\prime}+\frac{1}{2} q^{\prime}\right.\right. \\
& \left.+\frac{1}{2} q^{\prime \prime}, q^{\prime}+q^{\prime \prime}, t^{\prime}\right)-f^{(2)}\left(p-\frac{1}{2} q^{\prime}+\frac{1}{2} q^{\prime \prime}, q-q^{\prime}-q^{\prime \prime}, p^{\prime}\right. \\
& \left.\left.+\frac{1}{2} q^{\prime}-\frac{1}{2} q^{\prime \prime}, q^{\prime}+q^{\prime \prime}, t^{\prime}\right)\right] \\
& +\int_{-\infty}^{t} d t^{\prime} \exp \left[-i\left[\left(q-q^{\prime}\right) \frac{p+\frac{1}{2} q^{\prime}}{m}+q^{\prime} \frac{p^{\prime}-\frac{1}{2} q^{\prime}}{m}\right]\left(t-t^{\prime}\right)\right] \\
& \times\left[f ^ { ( 2 ) } \left(p+\frac{1}{2} q^{\prime}-\frac{1}{2} q^{\prime \prime}, q-q^{\prime}-q^{\prime \prime}, p^{\prime}-\frac{1}{2} q^{\prime}\right.\right. \\
& \left.+\frac{1}{2} q^{\prime \prime}, q^{\prime}+q^{\prime \prime}, t^{\prime}\right)-f^{(2)}\left(p+\frac{1}{2} q^{\prime}+\frac{1}{2} q^{\prime \prime}, q-q^{\prime}-q^{\prime \prime}, p^{\prime}\right. \\
& \left.\left.\left.-\frac{1}{2} q^{\prime}-\frac{1}{2} q^{\prime \prime}, q^{\prime}+q^{\prime \prime}, t^{\prime}\right)\right]\right\}
\end{aligned}
$$

where $V_{\text {eff }}$ (a functional of $f^{(1)}$ ) is defined in Eq. (4.23).

The expression involving $V_{\text {eff }}$ is easily identified with the "force" term $-\nabla V(\underline{R}, t) \cdot \nabla_{P} f^{(1)}(P, R, t)$ appearing in the usual Boltzmann equation. To see this, expand $f^{(1)}\left(p_{1} \pm \frac{1}{2} q_{1}\right)$ about $p_{1}$, getting

$$
\int \frac{d^{3} q^{\prime}}{(2 \pi)^{3}}\left[-q^{\prime} V_{\mathrm{eff}}\left(q^{\prime}, t\right)\right] \cdot \nabla_{p} f^{(1)}\left(\underline{p}, q-q^{\prime}, t\right)=-i \int d^{3} R e^{-i q \cdot \underline{R}}\left[\nabla V_{\mathrm{eff}}(R, t) \cdot \nabla_{p} f^{(1)}(p, R, t)\right],
$$

where in this case $V_{\text {eff }}$ is given by the second term of Eq. (4.23). Thus in $p R t$ variables the left-hand side has the familiar form

$$
i\left[\frac{\partial}{\partial t}+v \cdot \nabla_{R}-\nabla V_{\mathrm{eff}} \cdot \nabla_{p}\right] f^{(1)}(\underline{p}, R, t) .
$$

In order to manipulate the right-hand side of Eq. (4.33) to recognizable form, we work in $\omega$ space, where the time exponentials appear as energy denominators. We obtain

$$
\begin{aligned}
(N-1) \int \frac{d^{3} q^{\prime}}{(2 \pi)^{3}} \int \frac{d^{3} q^{\prime \prime}}{(2 \pi)^{3}} \int d^{3} p^{\prime} v\left(q^{\prime}\right) v\left(q^{\prime \prime}\right)\left(I_{1}+I_{2}\right), \\
I_{1}=\frac{1}{\omega-\left(q-q^{\prime}\right) \cdot \frac{\left(p-\frac{1}{2} q^{\prime}\right)}{m}-q^{\prime} \cdot \frac{\left(p+\frac{1}{2} q^{\prime}\right)}{m}} \\
\quad \times\left[f^{(2)}\left(p-\frac{1}{2} q^{\prime}-\frac{1}{2} q^{\prime \prime}, q-q^{\prime}-q^{\prime \prime}, p^{\prime}+\frac{1}{2} q^{\prime}+\frac{1}{2} q^{\prime \prime}, q^{\prime}+q^{\prime \prime}, \omega\right)\right. \\
\left.\quad-f^{(2)}\left(p-\frac{1}{2} q^{\prime}+\frac{1}{2} q^{\prime \prime}, q-q^{\prime}-q^{\prime \prime}, p^{\prime}+\frac{1}{2} q^{\prime}-\frac{1}{2} q^{\prime \prime}, q^{\prime}+q^{\prime \prime}, \omega\right)\right],
\end{aligned}
$$




$$
\begin{aligned}
I_{2}= & \frac{1}{\omega-\left(q-q^{\prime}\right) \cdot \frac{\left(p+\frac{1}{2} q^{\prime}\right)}{m}-q^{\prime} \cdot \frac{\left(p^{\prime}-\frac{1}{2} q^{\prime}\right)}{m}} \\
& \times\left[f^{(2)}\left(p+\frac{1}{2} q^{\prime}-\frac{1}{2} q^{\prime \prime}, q-q^{\prime}-q^{\prime \prime}, p^{\prime}-\frac{1}{2} q^{\prime}+\frac{1}{2} q^{\prime \prime}, q^{\prime}+q^{\prime \prime}, \omega\right)\right. \\
& \left.-f^{(2)}\left(p+\frac{1}{2} q^{\prime}+\frac{1}{2} q^{\prime \prime}, q-q^{\prime}-q^{\prime \prime}, p^{\prime}-\frac{1}{2} q^{\prime}-\frac{1}{2} q^{\prime \prime}, q^{\prime}+q^{\prime \prime}, \omega\right)\right] .
\end{aligned}
$$

At this state, it is not necessary to factorize $f^{(2)}$. However, it will be very helpful to first study the spatially homogeneous case in which

$$
\begin{aligned}
& f^{(1)}(p, q, \omega)=(2 \pi)^{3} \delta(q) f^{(1)}(p, \omega), \\
& f^{(2)}\left(p_{1} q_{1} p_{2} q_{2} \omega\right)=(2 \pi)^{3} \delta\left(q_{1}\right) \delta\left(q_{2}\right) f^{(2)}\left(p_{1} p_{2} \omega\right) .
\end{aligned}
$$

The integrand contains the common factor $\delta\left(q^{\prime}+q^{\prime \prime}\right) \delta(q)$. Canceling and simplifying leads to (dropping the Vlasov term here)

$$
\begin{aligned}
& \omega f^{(1)}(p, \omega)=(N-1) \int \frac{d^{3} q^{\prime}}{(2 \pi)^{3}}\left|v\left(q^{\prime}\right)\right|^{2} \int d^{3} p^{\prime}\left(I_{1}^{\prime}+I_{2}^{\prime}\right), \\
& I_{1}^{\prime}=\frac{1}{\omega+q^{\prime} \cdot \frac{\left(p-p^{\prime}\right)}{m}-\frac{q^{\prime 2}}{m}+i \varepsilon}\left[f^{(2)}\left(p, p^{\prime}, \omega\right)-f^{(2)}\left(p-q^{\prime}, p+q^{\prime}, \omega\right)\right], \\
& I_{2}^{\prime}=\frac{1}{\omega+q^{\prime} \cdot \frac{\left(p-p^{\prime}\right)}{m}+\frac{q^{\prime 2}}{m}+i \varepsilon}\left[f^{(2)}\left(p+q^{\prime}, p-q^{\prime}, \omega\right)-f^{(2)}\left(p, p^{\prime}, \omega\right)\right] .
\end{aligned}
$$

We now combine terms by setting $q^{\prime} \rightarrow-q^{\prime}$ in $I_{2}^{\prime}$. Noting the identity

$$
\Delta E \equiv E_{p}+E_{p^{\prime}}-E_{p-q^{\prime}}-E_{p^{\prime}+q^{\prime}}=\frac{q^{\prime} \cdot\left(p-p^{\prime}\right)}{m}-\frac{q^{\prime 2}}{m},
$$

we find

$$
\omega f^{(1)}(p, \omega)=(N-1) \int \frac{d^{3} q^{\prime}}{(2 \pi)^{3}}\left|v\left(q^{\prime}\right)\right|^{2} \int d^{3} p^{\prime}\left[\frac{1}{\Delta E+\omega+i \varepsilon}-\frac{1}{\Delta E-\omega-i \varepsilon}\right]\left[f^{(2)}\left(p, p^{\prime}, \omega\right)-f^{(2)}\left(p-q^{\prime}, p^{\prime}+q^{\prime}, \omega\right)\right]
$$

The energy difference in [Eq. (4.39)] has a clear pictorial significance in scattering theory. For a given $p, p^{\prime}, q^{\prime}$, this $\Delta E$ defines a collision time $\tau_{\text {coll }} \sim \hbar / \Delta E$. If $\tau_{\text {coll }}$ is much less than the characteristic time in which $f$ changes, then for significant portions of the integrand $\omega \ll \Delta E$ and the energy denominators produce an energy-conserving delta function $-2 \pi i \delta(\Delta E)$ of just the right character to yield the cross section necessary for the Boltzmann equation.

To make this argument more precise, we transform back from $\omega$ to $t$, getting

$$
\begin{array}{rl}
\frac{\partial f(p, t)}{\partial t}=-(N-1) \int \frac{d^{3} q^{\prime}}{(2 \pi)^{3}}\left|v\left(q^{\prime}\right)\right|^{2} \int d^{3} p^{\prime} \int_{-\infty}^{t} & d t^{\prime} e^{i(\Delta E)\left(t-t^{\prime}\right)}+e^{-i(\Delta E)\left(t-t^{\prime}\right)} \\
& \times\left[f^{(2)}\left(p, p^{\prime}, t^{\prime}\right)-f^{(2)}\left(p-q^{\prime}, p^{\prime}+q^{\prime}, t^{\prime}\right)\right] .
\end{array}
$$

Since $\Delta E \sim 1 / \tau$, if $f$ varies slowly on a time scale compared with $\tau$, regions of the integrand with $t-t^{\prime} \gg \tau$ contribute. So we evaluate $f^{(2)}$ at $t^{\prime} \approx t$ and do the time integrals directly. In this limit only $t^{\prime} \approx t$ matters: the system has lost its memory and becomes stochastic. The effective time integral is now $\int_{-\infty}^{\infty} e^{i \Delta E \tau} d \tau$, giving

$$
\frac{\partial f(p, t)}{\partial t}=(N-1) \int d^{3} p^{\prime} \int \frac{d^{3} q^{\prime}}{(2 \pi)^{3}}\left|v\left(q^{\prime}\right)\right|^{2} 2 \pi \delta(\Delta E)\left[f^{(1)}\left(p-q^{\prime}, t\right) f^{(1)}\left(p^{\prime}+q^{\prime}, t\right)-f^{(1)}(p, t) f^{(1)}\left(p^{\prime}, t\right)\right]
$$

which is the usual form of the homogeneous Boltzmann equation, on identifying

$$
v_{p-p^{\prime}} \frac{d \sigma}{d \Omega}=\int \frac{d^{3} q^{\prime}}{(2 \pi)^{3}}\left|v\left(q^{\prime}\right)\right|^{2} 2 \pi \delta(\Delta E)
$$

as the transition rate for $p+p^{\prime} \rightarrow\left(p-q^{\prime}\right)+\left(p^{\prime}-q^{\prime}\right)$. For bosons or fermions, obvious corrections need to be made to the occupation probabilities.

Intuitively, one expects that for a dilute gas the scatterings occur on-shell, and that in the above one can substi- 
tute $v \rightarrow t$, where $t$ describes the scattering to all orders in $v$. This supposition is encouraged by the fact that $\widetilde{f}^{(2)}$ obeys the full two-body scattering equation. For dense systems, one can expect significant energy shifts, off-shell, and lifetime effects. This aspect of the problem has been analyzed by Snider (1960) and others.

The classical collision time for a potential of finite range $a$ is given by $v \tau_{\mathrm{cl}} \approx a$, where $v$ is the relative velocity:

$$
\frac{1}{\tau_{\mathrm{cl}}} \approx \kappa\left|p-p^{\prime}\right| / m
$$

$\kappa \equiv 1 / a$ is the maximum momentum supplied by the potential. In contrast the quantum collision time determined by Eq. (4.39) is not given by $\kappa$ although since $\left|q^{\prime}\right|<\kappa$ there is a bound

$$
\frac{1}{\tau_{\text {coll }}} \leq \frac{\kappa\left|p-p^{\prime}\right|}{m}+\frac{\kappa^{2}}{m}
$$

For that set of collisions having $\left|p-p^{\prime}\right|>\kappa$ the upper bound of Eq. (4.45) coincides with $\tau_{\mathrm{cl}}$.

The relaxation time $\tau$ provides a key to the question of energy conservation. From Eq. (4.42) we can define a relaxation time, describing the scattering out of momentum states $p, p^{\prime}$ :

$$
\frac{1}{\tau}=-\frac{1}{f} \frac{\partial f}{\partial t}=\frac{N}{V} v \sigma
$$

for large $N$, with $V$ the volume and $\sigma$ the usual cross section. Equation (4.46) is equivalent to the traditional mean-free-path formula, $l=(\rho \sigma)^{-1}$ with $\rho=N / V$ the number density. From Eq. (4.46) we see that

$$
\left|\frac{\omega}{\Delta E}\right| \approx \frac{\tau_{\text {coll }}}{\tau},
$$

confirming the argument given after Eq. (4.40). Comparing Eq. (4.44) with Eq. (4.46) gives

$$
\frac{\tau_{\mathrm{coll}}}{\tau} \approx \frac{\alpha}{l} \approx \frac{\rho \sigma}{\kappa}
$$

For a dilute gas, $a / l$ is very small.

Several dimensional parameters play a role when we consider systems of finite size: for example, in a nucleus we have the interparticle distance and the surface thickness as well as the potential size as length parameters.

Baym and Kadanoff (1962) have discussed the form of the Boltzmann equation in the case of a slowly varying external potential. Under suitable circumstances, the net result is to consider Eq. (4.42) locally valid, i.e., each $f(p, t)$ is replaced by $f(p, R, t)$ with a common $R$. For applications to scattering problems as contemplated here, the inhomogeneities are much greater, so that genuine retardation effects must be retained. We now repeat in coordinate space a sequence of approximations similar to that leading to the spatial uniform Boltzmann equation (4.42). We leave in abeyance the question of the relative validity of the factorization approximation in the two cases.

The coordinate-space version of Eq. (4.14) is

$$
\begin{gathered}
{\left[\frac{\partial}{\partial t}+v_{1} \cdot \nabla_{1}\right] f\left(p_{1}, R_{1}, t\right)=-i \int d^{3} r_{1} e^{i\left(p_{1}^{\prime}-p_{1}\right) \cdot r_{1}} \sum_{j=2}^{N} \int d^{3} p_{1}^{\prime} d^{3} p_{j}^{\prime} d R_{j}\left[v\left(R_{1}+\frac{1}{2} r_{1}-R_{j}\right)-v\left(R_{1}-\frac{1}{2} r_{1}-R_{j}\right)\right]} \\
\times f_{2}\left(p_{1}^{\prime} R_{1} p_{2}^{\prime} R_{j} t\right)
\end{gathered}
$$

Our ansatz for $f_{2}$ corresponding to Eq. (4.30) is

$$
\begin{aligned}
& \tilde{f}_{2}\left(p_{1}^{\prime} R_{1} p_{2}^{\prime} R_{2} t\right)= f_{1}\left(p_{1}^{\prime} R_{1} t\right) f_{1}\left(p_{2}^{\prime} R_{2} t\right) \\
&-i \int_{-\infty}^{t} d t^{\prime} \int \frac{d^{3} p_{1}^{\prime \prime} d^{3} p_{2}^{\prime \prime}}{(2 \pi)^{3}} e^{i\left(p_{1}^{\prime \prime}-p_{1}\right) \cdot r_{1}^{\prime}} e^{i\left(p_{2}^{\prime \prime}-p_{1}^{\prime}\right) \cdot r_{2}^{\prime}} \widetilde{f}_{2}\left(p_{1}^{\prime \prime} R_{1}^{\text {ret }} p_{2}^{\prime \prime} R_{2}^{\text {ret }} t^{\prime}\right) \\
& \quad \times\left[v\left(R_{1}^{\text {ret }}+\frac{1}{2} r_{1}^{\prime}-R_{2}^{\text {ret }}-\frac{1}{2} r_{2}^{\prime}\right)-v\left(R_{1}^{\text {ret }}-\frac{1}{2} r_{1}^{\prime}-R_{2}^{\text {ret }}+\frac{1}{2} r_{2}^{\prime}\right)\right],
\end{aligned}
$$

with $R_{i}^{\text {ret }}=R_{i}-p_{i}^{\prime}\left(t-t^{\prime}\right) / m$.

Substituting this in Eq. (4.49), relabeling, and dropping the Vlasov term for simplicity, we obtain a generalization of Eq. (4.38),

$$
\begin{aligned}
& \frac{\partial f_{1}(p, R, t)}{\partial t}+v \cdot \nabla f_{1}(p, R, t) \\
& =-(N-1) \int \frac{d^{3} q_{1} d^{3} q_{2}}{(2 \pi)^{3}} d^{3} R^{\prime} d^{3} p^{\prime} \\
& \times \int_{-\infty}^{t} d t^{\prime} e^{i\left(q_{1}+q_{2}\right)\left(R-R^{\prime}\right)} v\left(q_{1}\right) v\left(q_{2}\right) \exp \left[-i q_{2} \frac{\left[p-p^{\prime}+\frac{1}{2}\left(q_{1}-q_{2}\right)\right]\left(t-t^{\prime}\right)}{m}\right] \\
& \times\left[\tilde{f}_{2}\left(p+\frac{1}{2}\left(q_{1}+q_{2}\right), R-\frac{p+\frac{1}{2} q_{1}\left(t-t^{\prime}\right)}{m}, p^{\prime}, R^{\prime}-\frac{p^{\prime}+\frac{1}{2} q_{2}\left(t-t^{\prime}\right), t^{\prime}}{m}\right)\right.
\end{aligned}
$$




$$
\begin{aligned}
& \left.-\widetilde{f}_{2}\left[p+\frac{1}{2}\left(q_{1}-q_{2}\right), R-\frac{p+\frac{1}{2} q_{1}\left(t-t^{\prime}\right)}{m}, p^{\prime}+q_{2}-\frac{p^{\prime}+\frac{1}{2} q_{1}\left(t-t^{\prime}\right), t^{\prime}}{m}\right]\right] \\
\times & \exp \left[-i q_{2} \frac{\left[p-p^{\prime}-\frac{1}{2}\left(q_{1}-q_{2}\right)\right]}{m}\right]\left(t-t^{\prime}\right) \\
\times & {\left[\widetilde{f}_{2}\left[p-\frac{1}{2}\left(q_{1}+q_{2}\right), R-\frac{p-\frac{1}{2} q_{1}}{m}\left(t-t^{\prime}\right), p^{\prime}, R^{\prime}-\frac{p^{\prime}-\frac{1}{2} q_{2}}{m}\left(t-t^{\prime}\right), t^{\prime}\right]\right.} \\
& \left.-\widetilde{f}_{2}\left[p-\frac{1}{2}\left(q_{1}-q_{2}\right), R-\frac{p-\frac{1}{2} q_{1}}{m}\left(t-t^{\prime}\right), p^{\prime}-q_{2}, R^{\prime}-\frac{p^{\prime}-\frac{1}{2} q_{2}}{m}\left(t-t^{\prime}\right), t^{\prime}\right]\right] .
\end{aligned}
$$

This result is only the spatial analog of Eq. (4.36), but is more amenable to approximations based on space-time intuition. For example, we can study the conditions under which Boltzmann's equation applies locally.

First note that when the distribution functions are uniform in the space coordinates, the retardation factors in the arguments of $f_{2}$ drop out, leaving a time integral which reproduces the energy denominators of Eq. (4.38). The reader can confirm by a straightforward calculation that Eq. (4.51) reduces to Eq. (4.38) in this limit. Note, in particular, that the $R$ independence of $\widetilde{f}_{2}$ yields $\delta\left(q_{1}+q_{2}\right)$, a necessary ingredient in producing the collision cross-section factor. As before, when $f$ varies slowly on the scale of a collision time, one gets the on-shell energy-conserving delta function.

Again, for slowly changing $f(t)$, the $t^{\prime}$ integration is restricted to a range $\Delta t=t-t^{\prime} \approx \alpha / v$ where $\alpha$ is the potential range [cf. Eq. (4.44)]. In that case, the retardation terms are negligible, i.e., $R \pm \alpha \approx R$, provided $f$ changes slowly over the distance $\alpha$. Now consideration of the exponential $\left(q_{1}+q_{2}\right) \cdot\left(R-R^{\prime}\right)$ shows that $R^{\prime}$ cannot differ by more than $\alpha$ from $R$, allowing us to write $R^{\prime} \simeq R$ in $f_{2}$. Finally, we obtain again $\delta\left(q_{1}+q_{2}\right)$ from the $R$ integration, which gives us the local version of Eq. (4.42) upon factorization.

At the surface of a nucleus, the retardation distance is of the same order as the surface thickness, and the local approximation will be wrong. Within the nucleus, the situation is better, insofar as $\alpha / R \approx 0.7 \alpha / A^{1 / 3}$ ( $\alpha$ in fermis). For $\alpha \approx 1.4 f, A=216$, we have $\alpha / R \approx 0.12$, but for $A=27, \quad \alpha / R \approx 0.33$, not negligible. Further, when bound-state effects are included (with oscillating phasespace densities), it is not clear whether the whole approach makes sense. Further research will be required to clarify this situation for realistic problems.

\section{Hydrodynamic equations}

As in the case of the one-particle potential problem, hydrodynamic equations follow immediately on taking suit- able moments of the equations of motion. It is significant to note that these formal structures are quite independent of boundary conditions or assumptions regarding local thermodynamic equilibrium. The deduction of hydrodynamic and transport equations has an extensive history, which is reviewed by Mori, Oppenheim, and Ross (1962) and de Groot and Suttorp (1972).

From the particle density

$$
\rho(R)=\sum_{i} \delta\left(\underline{R}-\underline{R}_{i}\right)
$$

we construct the averaged density

$$
\begin{aligned}
n(\underline{R}, t)=\prod_{i} \int & d^{3} p_{i} d^{3} R_{i} \\
& \times \sum_{\alpha} \delta\left(\underline{R}-\underline{R}_{\alpha}\right) f_{N}\left(p_{1} R_{1} \cdots p_{N} R_{N} t\right) .
\end{aligned}
$$

This definition, which treats all particles symmetrically, differs by a normalization factor $N$ from Eq. (4.9).

Imitating the calculations of Sec. II.G leads directly to the continuity equation [Eq. (128)], with

$$
\begin{aligned}
& n(\underline{R}, t) \underline{u}(\underline{R}, t) \equiv \prod_{i} \int d^{3} p_{i} d^{3} R_{i} \\
& \times \sum_{i} \frac{p_{i}}{m} f\left(p_{1} R_{1} \ldots p_{N} R_{N} t\right) \\
& \times \delta\left(R-R_{i}\right) .
\end{aligned}
$$

Multiplying the equation of motion with the average velocity

$$
\sum_{i} \frac{p_{i}}{m} \delta\left(R-R_{i}\right)
$$

and integrating over phase space gives the momentum equation analogous to Eq. (2.131): 


$$
\begin{aligned}
& i\left[\frac{\partial}{\partial t}(n \underline{u})\right]+\prod_{i} \int d^{3} p_{i} d^{3} R_{i} \sum_{j} \frac{p_{j}}{m} \cdot \nabla_{j} f \sum_{\alpha} \frac{p_{\alpha}}{m} \delta\left(\underline{R}-\underline{R}_{\alpha}\right) \\
& \quad=\prod_{i, j} \int d^{3} p_{i} d^{3} R_{i} d^{3} p_{j}^{\prime} d^{3} r_{j} e^{i\left(p_{j}^{\prime}-p_{j}\right) \cdot r_{j}} \sum_{\alpha} \frac{p_{\alpha}}{m} \delta\left(\underline{R}-\underline{R}_{\alpha}\right)\left(\sum_{i<j}\left[v\left(x_{i}^{\prime}-x_{j}^{\prime}\right)-v\left(x_{i}-x_{j}\right)\right]+\sum_{j}\left[V\left(x_{j}^{\prime}, t\right)-V\left(x_{j}, t\right)\right]\right) .
\end{aligned}
$$

Manipulation of the drift term leads to the analog of Eq. (2.136),

$$
i \frac{\partial}{\partial R_{i}} \int \Pi d^{3} p_{k} d^{3} R_{k} \sum_{\alpha} v_{\alpha}^{i} v_{\alpha}^{k} \delta\left(R-R_{\alpha}\right) f\left(p_{1} R_{1} \cdots p_{N} R_{N} t\right) \equiv i \frac{\partial}{\partial R_{i}}\left[n(R, t)\left\langle U^{i} U^{k}\right\rangle\right] .
$$

On the right-hand side the potential terms act as forces-one due to the external fields $V$ and the other due to all the particles other than the chosen one at point $\underline{R}$.

Explicitly we have for the right-hand side of Eq. (4.56):

$$
-\frac{i}{m} \sum_{\alpha} \int \prod d^{3} p_{k} d^{3} R_{k} \delta\left(R-R_{\alpha}\right)\left[\frac{\partial V}{\partial R_{\alpha}}\left(R_{\alpha}\right)+\sum_{\beta \neq \alpha} \frac{\partial v\left(R_{\alpha}-R_{\beta}\right)}{\partial R_{\beta}}\right] f\left(p_{1} R_{1} \cdots p_{N} R_{N} t\right) \equiv-i \frac{n(R, t)}{m}\left[\nabla V_{\mathrm{ex}}+\nabla V_{\mathrm{int}}\right]
$$

The equation of motion for the momentum density is then very similar to that for the one-particle result [Eq. (2.131)]:

$$
\frac{\partial}{\partial t}\left(n u_{i}\right)+\frac{\partial}{\partial R_{i}}\left(n\left\langle U_{i} U_{j}\right\rangle\right)=-\frac{n}{m}\left(\nabla V_{\mathrm{ex}}+\nabla V_{\mathrm{int}}\right)
$$

As before [Eq. (2.137)], one can manipulate Eq. (4.59) into the form of Euler's equation.

The existence of these formal structures in no way assumes local equilibrium or classical approximations. Naturally, the calculation of the quantities appearing in the equation requires a knowledge of the distribution functions, i.e., a solution of the $N$-body problem.

\section{E. Formulation in second quantization}

The correct description of the many-body system requires that close attention be paid to the particle statistics and the spin and internal symmetry (e.g., isospin) variables of the problem. The preceding development has ignored these cumbersome technical details in order to express more clearly the structural aspects of the theory. Although the structure of the $N$-body wave function has been much studied with regard to these questions, it is often expeditious to use the compact notation made possible by the formalism of "second quantization." The equivalence of this approach to the usual $N$-body Schrödinger equation is explained in textbooks by Huang (1963), Schweber (1961), and Fetter and Walecka (1971).

The dynamical coordinates in the Heisenberg picture $\left[\left(\psi(\underline{x}, t)\right.\right.$ and its adjoint $\left.\psi^{+}(\underline{x}, t)\right]$ obey the commutation or anticommutation rules,

$$
\begin{aligned}
& {\left[\psi_{\alpha}\left(\underline{x}, t, \psi_{\beta}^{+}\left(\underline{x}^{\prime}, t\right)\right]_{ \pm}=\delta_{\alpha \beta} \delta\left(\underline{x}-\underline{x}^{\prime}\right),\right.} \\
& {\left[\psi(\underline{x}, t), \psi\left(\underline{x}^{\prime}, t\right)\right]_{ \pm}=0,}
\end{aligned}
$$

for boson or fermion fields. Here $\alpha$ and $\beta$ denote labels for any discrete internal variables such as spin and isospin.

The generalized (matrix) Wigner function is then defined in terms of the density operator $\rho$ by

$$
\begin{aligned}
& f_{\alpha \beta}(\underline{p}, \underline{R}, t) \\
& \quad \equiv \int \frac{d^{3} t}{(2 \pi)^{3}} e^{-\underline{i} \underline{p} \cdot \underline{\underline{r}}} \operatorname{Tr}\left[\rho \psi_{\alpha}^{+}\left(\underline{R}-\frac{1}{2} \underline{r}, t\right) \psi_{\beta}\left(\underline{R}+\frac{1}{2} \underline{r}, t\right)\right] .
\end{aligned}
$$

For a pure state involving the wave function $|\psi\rangle$, we have $\rho=|\Psi\rangle\langle\Psi|$. In particular, for a one-particle state this is completely equivalent to the definition (2.92) (except the order of indices, which is a matter of convention).

The total number density is now

$$
\begin{aligned}
\sum_{a} \int d^{3} p f_{\alpha \alpha}(\underline{p}, \underline{R}, t) & =\operatorname{Tr} \rho \psi^{+}(\underline{R}, t) \psi(\underline{R}, t) \\
& \equiv\left\langle\psi^{+}(\underline{R}, t) \psi(\underline{R}, t)\right\rangle .
\end{aligned}
$$

If we expand $\psi$ in the momentum basis as in Eq. (2.2), with $C(\underline{p}, t) \rightarrow a_{\alpha}(\underline{p}, t)$ being destruction operators obeying

$$
\begin{aligned}
& {\left[a_{\alpha}(\underline{p}, t), a_{\beta}^{+}\left(\underline{p}^{\prime}, t\right)\right]_{ \pm}=\delta_{\alpha \beta} \delta\left(\underline{p}-\underline{p}^{\prime}\right),} \\
& {\left[a_{\alpha}(\underline{p}, t), a_{\beta}\left(\underline{p}^{\prime}, t\right)\right]_{ \pm}=0,}
\end{aligned}
$$

we find the alternative form

$$
f_{\alpha \beta}=\int \frac{d^{3} q}{(2 \pi)^{3}} e^{-i q \cdot \underline{R}} \operatorname{Tr}\left[\rho a_{\alpha}^{+}\left(p+\frac{1}{2} q, t\right) a_{\beta}\left(p-\frac{1}{2} q, t\right)\right] \text {. }
$$

The pair operator $a_{p+q}^{+} a_{p}$ is useful in describing density fluctuations and plays a prominent role in the many-body problem.

Integrating over phase space gives $\left(d \Gamma \equiv d^{3} p d^{3} R\right)$ 


$$
\int d \Gamma \sum_{a} f_{\alpha \alpha}(\underline{p}, \underline{R}, t)=\operatorname{Tr} \rho N_{\mathrm{op}}=\langle N\rangle,
$$

where $N_{\text {op }}$ is

$$
N_{\mathrm{op}}=\sum_{\alpha} \int d^{3} p a_{\alpha}^{+}(\underline{p}, t) a_{\alpha}(\underline{p}, t)
$$

For number eigenstates $\langle N\rangle=N$; hence the natural normalization here differs from that used before.

In defining higher-order distribution functions, the question of operator ordering immediately arises. The best choice seems to be a suitably normal ordered form; for the two-particle distribution this is

$$
f_{\alpha \beta, \gamma \delta}^{(2)}\left(p_{1} R_{1}, p_{2} R_{2}, t\right) \equiv \int \frac{d^{3} r_{1} d^{3} r_{2}}{(2 \pi)^{3}} d^{-i p_{1} \cdot r_{1}-i p_{2} \cdot r_{2}}\left\langle\psi_{\alpha}^{+}\left(R_{1}-\frac{1}{2} r_{1}, t\right) \psi_{\gamma}^{+}\left(R_{2}-\frac{1}{2} r_{2}, t\right) \psi_{\delta}\left(R_{2}+\frac{1}{2} r_{2}, t\right) \psi_{\beta}\left(R_{1}+\frac{1}{2} r_{1}, t\right)\right\rangle,
$$

where, as usual, the average bracket $\langle 0\rangle \equiv \operatorname{Tr} \rho 0$. This definition is suitable for both boson and fermion fields.

We note the reality condition obeyed by Eq. (4.67):

$$
f_{\alpha \beta, \gamma \delta}^{*}\left(p_{1} R_{1} p_{2} R_{2} t\right)=f_{\beta \alpha, \delta \gamma}^{(2)}\left(p_{1} R_{1} p_{2} R_{2} t\right) \text {. }
$$

Integration over the two-particle phase space gives

$$
\int d \Gamma_{1} d \Gamma_{2} f_{\alpha \beta, \gamma \delta}^{(2)}=\left\langle N_{\alpha \beta}(t) N_{\gamma \delta}(t)\right\rangle-\delta_{\gamma \beta}\left\langle N_{\alpha \delta}(t)\right\rangle .
$$

Tracing out the internal variables gives

$$
\int d \Gamma_{1} d \Gamma_{2} \sum_{\alpha, \gamma} f_{\alpha \alpha, \gamma \gamma}^{(2)}=\left\langle N^{2}\right\rangle-\langle N\rangle,
$$

reducing to $N(N-1)$ for number eigenstates.

The suitability of the definition (4.67) becomes clear when we examine the Hamiltonian and the equation of motion. In second quantization the analog of Eq. (4.1) extended to include internal degrees of freedom (but with a common external potential) is

$$
H=\int d^{3} x \psi_{\alpha}^{+}(x)\left(\frac{-\nabla^{2}}{2 m}\right) \psi_{\alpha}(x)+\int d^{3} x \psi_{\alpha}^{+}(x) V_{\alpha \beta}(\underline{x}, t) \psi_{\beta}(x)+\frac{1}{2} \int d^{3} x d^{3} x^{\prime} \psi_{\alpha}^{+}(x) \psi_{\beta}^{+}\left(x^{\prime}\right) v_{\alpha \beta, \gamma \delta}\left(\underline{x}-\underline{x}^{\prime}\right) \psi_{\delta}\left(x^{\prime}\right) \psi_{\beta}(x)
$$

Hermiticity requires $V_{\alpha \beta}^{*}=V_{\beta \alpha}$ and $v_{\beta \alpha, \delta \gamma}^{*}=v_{\alpha \beta, \gamma \delta}$. Here we have dropped the time label and adopted the summation convention.

An elementary calculation now gives the theorem

$$
\begin{aligned}
\langle H\rangle= & \int d \Gamma\left[\frac{p^{2}}{2 m} \delta_{\alpha \beta}+V_{\alpha \beta}(R, t)\right] f_{\alpha \beta}^{(1)}(p, R, t) \\
& +\frac{1}{2} \int d \Gamma_{1} d \Gamma_{2} v_{\alpha \beta, \gamma \delta}\left(R_{1}-R_{2}\right) f_{\alpha \beta, \gamma \delta}^{(2)}\left(p_{1} R_{1} p_{2} R_{2} t\right) .
\end{aligned}
$$

This result extends the usual theorems for the groundstate energy (Fetter and Walecka, 1971). The latter expressions involve boundary values of Green's functions referred to the ground state. These boundary values actually serve to represent the physical densities given by the Wigner distributions. Expressions of the $N$-body dynamics via the Green's-function method has, of course, the advantage of providing the Feynman graph analysis of the problem. In many cases, one will want to supplement the Wigner method by using these well-developed techniques. The equation of motion involving all the indices is a straightforward extension of Eq. (4.20), whose derivation we leave to the reader.

\section{F. Effect of spin and statistics}

Except in Sec. IV.E, we have treated the particles as spinless and distinguishable but otherwise identical.
Often this is referred to as "Maxwell" statistics. In this case, the labels are attached to definite particles, and, for example, the one-particle distribution

$$
f\left(p_{1} R_{1} t\right)=\int \prod_{k=2}^{N} d \Gamma_{k} f_{N}\left(p_{1} R_{1} \cdots p_{N} R_{N} t\right)
$$

is specific to particle 1 . Since the Hamiltonian is symmetrical in the $N$ particles, we expect that the $f\left(p_{i}, R_{i}, t\right)$ all have the same functional form except when external boundary conditions and potentials may intervene to make the distributions distinct.

For questions not concerning which particle is involved, we may define a generic distribution function which tells whether any of the $N$ particles is at $(p, R)$ in phase space. Introducing the one-particle density in phase space by

$$
\begin{aligned}
\rho & =\sum_{t=1}^{N} \delta\left(\underline{R}-\underline{R}_{i}\right) \delta\left(\underline{p}-\underline{p}_{i}\right) \\
& \equiv \sum_{i} \delta\left(\Gamma-\Gamma_{i}\right),
\end{aligned}
$$

we define the one-particle distribution

$$
f(p, R, t)=\int \prod_{k=1}^{N} d \Gamma_{k} \sum_{i} \delta\left(\Gamma-\Gamma_{i}\right) f_{N} .
$$

In the absence of spin effects, $f_{N}$ is symmetrical under the interchange $\left(p_{i} R_{i}\right) \leftrightarrow\left(p_{k}, R_{k}\right)$ for bosons or fermions, and so each particle contributes equally. Then Eq. (4.75) 
differs only by a normalization factor $N$ from Eq. (4.73), as in the Maxwell case.

Of course, the true wave function typically contains spin variables (and perhaps others) in addition to the space coordinates. Then $f$ must be labeled suitably to handle the spin coordinates. Ordinarily, the wave function will be decomposed into irreducible representations of the permutation group in the form of products of spin and space functions of possibly rather complicated mixed symmetries. In some cases, the spin complications may be significant, each problem being treated on its own merits.

The simplest example is a two-particle fermion system composed of one-particle states,

$$
\psi=\frac{1}{\sqrt{2}}\left[u_{i}(1) u_{j}(2)-u_{i}(2) u_{j}(1)\right],
$$

where the labels $i$ and $j$ indicate the one-particle-state quantum numbers. We take $i \neq j$, since otherwise $\psi$ vanishes. For simplicity, let $u_{i}$ be energy eigenstates carrying spin $\frac{1}{2}$. The two-particle distribution function is

$$
\begin{aligned}
f_{i j}\left(p_{1} R_{1} p_{2} R_{2}\right)= & f_{i i}\left(p_{1} R_{1}\right) f_{j j}\left(p_{2} R_{2}\right) \\
& +f_{i i}\left(p_{2} R_{2}\right) f_{j j}\left(p_{1} R_{1}\right) \\
& -f_{i j}\left(p_{1} R_{1}\right) f_{j i}\left(p_{2} R_{2}\right) \\
& -f_{i j}\left(p_{2} R_{2}\right) f_{j i}\left(p_{1} R_{1}\right) .
\end{aligned}
$$

Integration according to the rule (4.75) gives

$$
f(p, R)=f_{i i}(p, R)+f_{j j}(p, R) .
$$

We can, of course, decompose the wave functions according to spin 0 and spin 1, i.e., symmetric or antisymmetric space states,

$$
\begin{aligned}
& \Psi_{0}=\frac{1}{\sqrt{2}}\left[\varphi_{\alpha}\left(x_{1}\right) \varphi_{b}\left(x_{2}\right)+\varphi_{b}\left(x_{1}\right) \varphi_{a}\left(x_{2}\right)\right] \chi^{S=0} \\
& \Psi_{M}^{1}=\frac{1}{\sqrt{2}}\left[\varphi_{a a}\left(x_{1}\right) \varphi_{b}\left(x_{2}\right)-\varphi_{b}\left(x_{1}\right) \varphi_{a}\left(x_{2}\right)\right] \chi_{M}^{S=1}
\end{aligned}
$$

In analogy to the one-particle case (2.125), we write a Wigner function in the coupled spin basis

$$
f_{\underline{M} \underline{M}^{\prime}}^{S S^{\prime}} \sim \int \Psi_{S^{\prime} \underline{M}^{\prime}}^{+} \Psi_{S \underline{M}} .
$$

Tracing on the spin components gives $\delta_{S S^{\prime}}$ and independent Wigner functions for each spin. Of course, the potential may couple the $f^{S}$ in such a way as to require the full matrix set (4.80). The basis (4.80) [more general than the example wave function of (4.79)] is generally the most useful in practical problems.

Next consider the one-particle distribution for a Slater determinant of one-particle functions (Moyal, 1949; de Groot and Suttorp, 1972):

$$
\Psi=\frac{1}{(N !)^{1 / 2}} \sum_{P}(-1)^{P} P u_{i}(1) u_{j}(2) \cdots u_{P}(N),
$$

where the subscripts label the occupied single-particle states (all distinct by the antisymmetrization). Integration according to Eq. (4.75) immediately leads to

$$
f(p, R, t)=\sum_{i} f_{i i}(p, R, t)
$$

where $f_{i i}$ is the one-particle Wigner function for state $i$. Equation (4.82) corresponds to representing the $N$-particle density matrix as the sum of one-particle density matrices.

The one- and two-particle distributions for Maxwell, Bose, and Fermi product wave functions are given in Chap. VII of de Groot and Suttorp (1972).

A semiclassical evaluation of the Wigner function for a one-dimensional Fermi gas in a potential well has been given by Balazs and Zipfel (1973). Shell-model calculations involving a "smeared" Wigner function have been presented by Baker, McCarthy, and Porter (1960). Comparison with the Thomas-Fermi model has been made in both these works.

\section{POSSIBLE APPLICATIONS IN INTERACTIONS OF COMPOSITE SYSTEMS}

\section{A. Composite particle scattering in the Liouville formalism}

The enormous variety of possible final-state channels, and the complexity of the time evolution leading to those final states, require a cumbersome formal apparatus reviewed by Goldberger and Watson (1964) and Newton (1966) for the usual Hamiltonian formalism. The additional complications caused by particle identity and statistics will be generally ignored in our outline. A cartoon of the physical situation is given in Fig. 9. We envision the collision of two bound systems having $N_{1}, N_{2}$ particles. Figure (9b), suitable for short-range forces, indicates interaction in the overlapping region. Following an extensive interaction in the collision volume [Fig. 9(c)], the system separates into final states comprising all imaginable bound-state configurations [Fig. 9(d)]. In describing these final states, it is traditional to speak of "channels" and "channel Hamiltonians" in which interactions between constituents in distinct bound states are ignored. A given channel involves a partition of the total number of final bound particles $N=N_{\alpha_{1}}^{+} N_{\alpha_{2}}+\cdots+N_{\alpha_{k}}$, with the individual final particles labeled by $\alpha_{i}$. Each of these partitions corresponds to an asymptotic wave function $\Phi_{\alpha}$, with the total wave function given by

$$
\Psi \underset{t \rightarrow \infty}{\rightarrow} \sum_{\alpha} C_{\alpha} \Phi_{\alpha}
$$

There is some model dependence tacit, in that an excited state with lifetimes greater than the transit time to the detector will count as a "bound state."

We should also note the expression of translation invariance, which allows one to factor out the overall $\mathrm{cm}$ motion in direct extension of the two-body problem, as in Eq. (3.11)ff. Introducing total and relative coordinates by 


$$
\begin{aligned}
& X=\frac{1}{N}\left(x_{1}+x_{2}+\cdots x_{N}\right), \\
& x_{12}=x_{1}-x_{2}, \\
& x_{23}=x_{2}-x_{3}, \\
& \vdots \\
& x_{N-1, N}=x_{N-1}-x_{N},
\end{aligned}
$$

and factoring out the $\mathrm{cm}$ motion as

$$
\Psi=\chi(X, t) \Phi\left(x_{i j}, t\right)
$$

leads to the expression for the Wigner function:

$$
f_{N}\left(p_{1} R_{1} \cdots p_{N} R_{N} t\right)=f_{0}(\underline{P}, \underline{R}, t) f_{N}\left(p_{i j}, R_{i j}, t\right),
$$

where the variables are

$$
\begin{aligned}
& P=\sum_{i=1}^{N} P_{i}, \\
& R=\sum_{i=1}^{N} R_{i} / N, \quad r=\sum r_{i} / N, \\
& R_{j}=R_{i}-R_{j}, \quad r_{i j}=r_{i}-r_{j}, \\
& p_{i j}=\left(p_{i}-p_{j}\right) / N,
\end{aligned}
$$
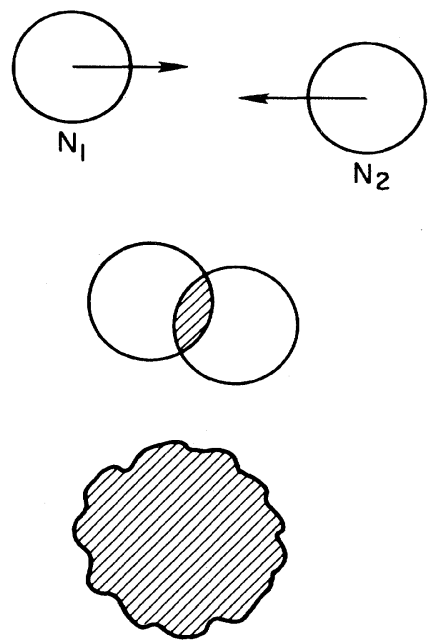

FIG. 9. Simplified version of the stages of a nucleus-nucleus collision. In (a) the incident density is $f_{N_{1}}^{(0)} f_{N_{2}}^{(0)}$. In (b) the nuclei overlap, initiating a kinetic phase (c), prior to the separation (d). and we have used the identity

$$
\sum_{i=1}^{N} \underline{r}_{i} \cdot \underline{p}_{i}=\underline{r} \cdot \underline{P}+\frac{1}{2} \sum_{i, j=1}^{N} \underline{r}_{i j} \cdot \underline{p}_{i j} .
$$

In the continuum limit $f_{0}$ reduces to $\delta\left(\sum p_{i}-P_{\text {tot }}\right)$ [cf. Eq. (2.17)] and the internal motion is described by the correct number of variables. Often we shall leave the overall momentum conservation tacitly understood.

Next consider the asymptotic Wigner function corresponding to the wave function (5.1). Each constituent $\Phi_{\alpha_{i}}$ is composed of a wave packet peaked at velocity $V_{\alpha_{i}}$ and an internal wave function. For large times the (finite) wave packets are nonoverlapping, so that the off-diagonal parts of the Wigner function vanish, giving

$$
f_{N}(-\infty)=f_{N_{1}}^{0} f_{N_{2}}^{0} \rightarrow \sum_{\alpha} \xi_{\alpha} f_{\alpha}(\infty),
$$

where $\xi_{\alpha}=\left|C_{\alpha}\right|^{2}$ is a function of the $\underline{P}_{\alpha}$ and

$$
f_{\alpha}=\prod_{\alpha_{i}} f_{\alpha_{i}} .
$$

Each $f_{\alpha_{i}}$ has as a factor its $\mathrm{cm}$ packet peaked at $P_{\alpha_{i}}^{0}$ and a stationary bound-state distribution describing the internal motion. Here the $P_{\alpha_{i}}$ are the overall momenta of bound state $\alpha_{i}$ :

$$
P_{\alpha_{i}}=\sum_{i \subset \alpha_{i}} P_{i}
$$

characterizing the free final packet $f_{0}^{\alpha_{i}}\left(R_{\alpha_{i}}-V_{\alpha_{i}} t\right)$ when

$$
R_{\alpha_{i}}=\sum_{i \subset \alpha_{i}} R_{i}
$$

In order to obtain the exclusive differential cross section for channel $\alpha$ due to an individual component of Eq. (5.7), we fix $\underline{P}_{\alpha_{1}} \cdots \underline{P}_{\alpha_{k}}$ and integrate over the cm positions $\underline{R}_{\alpha_{i}}$ and internal coordinates $\Gamma_{\alpha_{i}}$ of the final fragments, at $t \rightarrow \infty$ :

$$
\begin{aligned}
& \frac{d N_{\text {scatt }}}{d^{3} p_{\alpha_{1}} \cdots} d^{3} p_{\alpha_{k}} \\
& \quad=\prod_{i} \xi_{\alpha}\left(P_{\alpha_{i}}\right) \int \prod_{i} d^{3} R_{a_{i}} \prod_{i} \int d \Gamma_{\alpha_{i}}^{f} f_{\alpha_{i}}(t \rightarrow \infty) .
\end{aligned}
$$

By normalization the internal factors integrate to unity, and the overall motion due to the packets is peaked around $P_{\alpha_{i}}^{0}$. [For the Gaussian example, each factor gives $\delta\left(P_{\alpha_{i}}-P_{\alpha_{i}}^{0}\right) /(2 \pi)^{3}$ in the continuum limit.]

The foregoing analysis is, of course, only intended to expose the kinematical content of the phase-space approach. No panacea is recommended for the calculation of the dynamical information contained in $\xi_{\alpha}$, which is essentially the square of the $S$ matrix for production of channel $\alpha$. In principle, the $\xi_{\alpha}$ are found by propagating the initial density $f_{N_{1}}^{0} f_{N_{2}}^{0}$ for a long enough time and picking off the coefficients of an expansion in the chan- 
nels $f_{\alpha_{i}}$.

In the formal analysis of the channel dynamics, it is useful to define various Liouville operators. For a given subsystem $\alpha_{i}$, we have

$$
\begin{aligned}
L_{a_{i}} f_{\alpha_{i}} \equiv & -i \sum_{i \subset \alpha_{i}} v_{i} \cdot \nabla_{i} f_{\alpha_{i}} \\
& +i \int d^{3} p_{\alpha_{1}}^{\prime} \cdots d^{3} p_{\alpha_{k}}^{\prime} K_{\alpha_{i}}\left(p_{\alpha_{i}}^{\prime}-p_{\alpha_{i}}, R_{\alpha_{i}}\right) f_{\alpha_{i}},
\end{aligned}
$$

where $K_{\alpha_{i}}$ is computed from the potentials $v_{i j}$ acting among the particles in the fragment $\alpha_{i}$. The corresponding interacting evolution operator is

$$
i D_{\alpha_{i}} \equiv i \frac{\partial}{\partial t}+L_{\alpha_{i}},
$$

where $f_{\alpha_{i}}$ obeys

$$
D_{\alpha_{i}} f_{\alpha_{i}}=0 \text {. }
$$

[The time dependence of Eq. (5.14) is contained in the cm motion of the fragment $\alpha_{i}$.]

Corresponding to the channel $\alpha$ we write in analogy

$$
\begin{aligned}
& L_{\alpha} \equiv \sum_{\alpha_{i}} L_{\alpha_{i}}, \\
& i D_{\alpha} \equiv i \frac{\partial}{\partial t}+L_{\alpha},
\end{aligned}
$$

and note the identity

$$
D_{\alpha} \prod_{\alpha_{i}} f_{\alpha_{i}}=\prod_{\alpha_{i}}\left(D_{\alpha_{i}} f_{\alpha_{i}}\right) .
$$

The detailed analysis of the complicated dynamics of rearrangements, etc., is left to another occasion. We note, however, the papers of Remler (1981) and Hoffman, Kouri, and Top (1979) dealing with similar problems using the density-matrix formalism.

\section{B. Models of nucleus-nucleus collisions}

Contemporary interest in this subject centers on heavyion collisions, which often are conducted in a regime sufficiently relativistic to require extensions of the formalism thus far developed. In addition, creation of secondary quanta (usually pions) lies outside the usual Schrödinger formalism but is conveniently treated using the relativistic second-quantized version of the phase-space distribution, discussed briefly in Sec. VI.

The description of these collisions is generally couched in classical geometry, suitably modified by statistics. The main approaches are:

(1) intranuclear cascade models,

(2) classical equation of motion,

(3) hydrodynamical models,

(4) statistical phase-space models, and

(5) hot spot models.
Clearly, each of these topics bears reexamination in the light of the phase-space dynamical framework developed in this paper. Further discussion of the standard models, along with extensive references, may be found in the review of Gyulassy (1980).

A related topic much in need of clarification is the detailed mechanism of bound-state formation in the separation phase of the collision. For mass-shell particles in collision, the intervention of one or more other particles is required to satisfy energy-momentum conservation. Present models, particularly for deuteron production, have been assessed by Kapusta (1980). Hopefully the ad hoc character of these models can be reduced by an application of the present formalism, which combines quantum evolution with kinetic behavior in a convenient way.

In order to place these topics in proper perspective, it is useful to analyze the length and time scales relevant in a typical nuclear collision. (Throughout we have in mind short-range forces only.) The lengths are:

(a) nuclear radius $R$;

(b) nuclear surface thickness $\delta$;

(c) potential radius $a$;

(d) nucleon mean-free path $l$;

(e) Fermi wavelength $\lambda_{F}=\hbar / P_{F}$.

Corresponding to these lengths, we find characteristic times by dividing by a typical velocity $V$,

$$
\begin{aligned}
& \left(\mathrm{a}^{\prime}\right) \tau_{\mathrm{coll}} \equiv R / V ; \\
& \left(\mathrm{b}^{\prime}\right) \tau_{\mathrm{pen}} \equiv \delta / V ; \\
& \left(\mathrm{c}^{\prime}\right) \tau_{\mathrm{rel}} \equiv l / V ; \\
& \left(\mathrm{d}^{\prime}\right) \tau_{\mathrm{int}} \equiv \alpha / V ; \\
& \left(\mathrm{e}^{\prime}\right) \tau_{F} \equiv \hbar / V_{F} P_{F} .
\end{aligned}
$$

Here coll refers to the entire collision time of a nucleon interacting with the nucleus, $\tau_{\text {pen }}$ is the penetration time going from vacuum to the nuclear interior, $\tau_{\text {rel }}$ is the relaxation time during which the distribution function changes appreciably. Note that at low energies, $l$ (and $\tau_{\text {rel }}$ ) will be greatly enhanced over the interparticle distance because of Pauli blocking. Finally $\tau_{\text {int }}$ measures the time of a typical collision. In Sec. IV.C we already noted how $\tau_{\text {int }} \ll<\tau_{\text {rel }}$ was required in order to have nearly on-shell collisions necessary for a standard Boltzmann collision term. Further, we found that retardation terms could be dropped whenever $f_{1}$ changes slowly over a distance $\alpha$, leading to a "local" Boltzmann equation. Finally, if the relaxation rate $1 / \tau_{\text {rel }}$ of the medium is fast compared with the collision frequency $1 / \tau_{\text {coll }}$, we have local equilibrium and hence hydrodynamics supplemented by an equation of state. The statistical model can be regarded as an extreme limit of the hydrodynamical model, in which equilibrium extends over the entire interacting system prior to dissociation.

The Fermi wavelength refers to particles at the top of the Fermi sea and is relevant to the possibility of classical description of the motion. The Fermi "frequency" 
$1 / \tau_{F}=2 E_{F} / \hbar$ measures the rate of transfer of information inside the nucleus (quantal as opposed to the hydrodynamical time $R / v_{\text {sound }}$ with $v_{\text {sound }}$ computed from the equation of state: $\left.v^{2}=\partial P / \partial \rho\right) . \tau_{F}$ is typically of the order of $10^{-23} \mathrm{sec}$.

For fairly fast nucleons of several hundred $\mathrm{MeV}$, we have a mean free path $1 \sim 1 / \rho \sigma \sim 2 \mathrm{fm}$, and for $A \sim 10^{2}$

$$
R \approx 10 \mathrm{fm}, 1 \approx 2 \mathrm{fm}, \alpha \approx 1 \mathrm{fm},
$$

giving the inequalities

$$
\tau_{\text {int }}<\tau_{\text {rel }}<\tau_{\text {coll }}
$$

If the "less thans" were replaced by "much-less-thans," we would have confidence in most of the foregoing approaches, at least for portions of the overall reaction. At low energies, $l$ increases to the point that relaxation cannot occur within the interaction region, ruling out local thermodynamic equilibrium.

As shown in Sec. IV.D, the continuity equation and Euler's equation hold formally in $N$-particle systems independently of the assumption of local thermal equilibrium. However, these equations are not dynamically complete unless supplemented by further moment equations, or the traditional assumption of an equation of state (and hence equilibrium). It may be important to understand which hydrodynamic results depend crucially on equilibrium and which are implied by the structural character of the two basic hydrodynamic equations (2.128) and (4.59).

The hydrodynamic model of heavy-ion collisions has been studied by many authors. A review of this approach has been given by Nix (1979). Many further references are given in this work. Nix and Strottman (1981) have reassessed these results on the basis of newer data.

The traditional intranuclear cascade model (Metropolis et al., 1958) is essentially a billiard-ball model refined by the use of experimental cross sections and implemented by Monte Carlo techniques. There are obscurities connected with Fermi motion and the self-consistent binding potential. Quantum effects of the colliding wave packets and reflection as particles enter the potential well are usually neglected. An extensive treatment of the multiple scattering theory and Monte Carlo cascade theory has been developed in the Wigner formalism by Remler (1975) and Remler and Sathe (1978).

As mentioned before, the initial and final states involve bound systems, and non-negligible correlations exist, in contrast to fluidlike situations, where one- and twoparticle distribution functions often suffice. However, in the region of overlap shown in Figs. 9(b) and 9(c), it is quite plausible to use the Vlasov (TDHF)-Boltzmann approach of Sec. IV. Various models suggest themselves in order of increasing complexity.

(1) Quantum-mechanical evolution of the initial Wigner distribution

$$
f_{N_{1}}^{0} f_{N_{2}}^{0},
$$

using the best available wave functions for the nuclei.
The overlapping, interacting system can then be evolved in time up to the point of separation according to

(2a) classical evolution (implemented by the Boltzmann equation, treated by Monte Carlo methods), or

(2b) quantum Boltzmann evolution (as developed here, the only differences are the use of the quantum cross section unless retardation effects are taken into account), or

(2c) allowing the one-particle motions in the nucleus (taken from the TDHF equations) to collide by action of the Boltzmann equation.

At separation time, one then has a product of oneparticle distributions

$$
f_{N}\left(t_{\mathrm{sep}}\right)=\prod_{i=1}^{N} f_{i}\left(p_{i}, R_{i}, t_{\mathrm{sep}}\right) .
$$

(3) Coalescence into final bound states. As mentioned earlier, this part of the reaction needs further clarification.

(4) Quantum evolution of final-state wave packets for $t>t_{\text {sep }}$.

A few remarks on this rough sketch are in order. The motions of a sharply defined interaction region and separation time need to be carefully considered. For central collisions of equal-size objects, these concepts are reasonable, whereas for peripheral collisions a more sophisticated approach may be required. The separation time probably has to be defined in terms of the mean interparticle separation in a possibly reaction-dependent way. For example, if some particles are instantly blown off, they may have traveled some distance before the rest of the system dissociates.

Classical evolution of part of (or even the entire) reaction has been used with success in chemical physics (see references in the following subsection) and in nuclear physics (Bodmer and Panos, 1977; Wilets, Henley, Kraft, and MacKellar, 1977; Callaway, Wilets, and Yariv, 1979). We note that a classical description will be spoiled by Fermi-Pauli effects if the collision energy is too low. Since $E_{f} \approx 50 \mathrm{MeV}$, we might expect to apply classical methods in the range $100-300 \mathrm{MeV}$ particle $^{-1}$, cutting off at the higher end where particle production sets in.

The intermediate evolution described in (2c) above is clearly very ambitious and may exceed present computational strength. Even within TDHF the evolution is extremely complicated. Köhler and Flocard (1979) have studied the one-dimensional TDHF dynamics of colliding slabs and calculated the phase-space flow of the Wigner function. This example is already rather complicated. Another instructive one-dimensional example is given by Richert, Brink, and Weidenmüller (1979), who compute the modification of the Wigner distribution caused by the addition of an ad hoc collision term.

Particle transport in nuclei from the density-matrix and phase-space point of view has also been studied by Remler and Sathe (1978) and Thies (1979). Other kinetic approaches have been studied and reviewed by Weidenmüller (1980).

Finally we mention the "hot spot" models recently 
studied by Weiner and collaborators (Weiner, 1978; Stelte and Weiner, 1981). Local deposition of energy can be expected to occur, for example, in glancing collisions of nuclei. At present, the energy propagation from the excited hot spot is treated as a diffusive heat conduction process. In the usual way of thinking, this requires local thermodynamic equilibrium. Even so, the general ideas and qualitative phenomenology should survive more refined approximations.

\section{Atomic and molecular collisions}

Studies of chemical reaction theory and pure quantum theory have led to analyses of semiclassical, or just classical, approaches to scattering and bound-state problems. The classical Liouville appoach to potential scattering is discussed by Prigogine (1959); the formal structure of this approach is analyzed in detail by Miles and Dahler (1970) in close analogy to the quantum treatment of the scattering problem. For a discussion of the three-body problem, we refer the reader to $\mathrm{Eu}$ (1971).

There exist many more investigations focusing on the semiclassical limit, for example, the review of Miller (1974) emphasizing transformation theory and the WKB method, and the path-integral methods of Laing and Freed (1975) and others, whereby $\mathrm{cm}$ coordinates of $R(t)$ of molecules become semiclassical orbits, with internal motion remaining purely quantum mechanical. These lines of research have merged with the Wigner phasespace-distribution approach in the hands of Heller (1976, 1977) and others. In the case of bound states, the investigations of Berry and collaborators are especially to be noted (Berry, 1977; Berry and Balazs, 1979).

\section{Solid-state physics}

The tradition of kinetic equations and collective motions is very deeply rooted in condensed matter physics. We have already described in Sec. IV.C the Vlasov equation for a Coulomb gas treated in this context. We also mention the books of Kadanoff and Baym (1962) and Klimontovich (1967).

There has been little emphasis on the Wigner function per se in the many-body problem, largely due to the large number of equivalent and equally effective alternate formalisms. An especially nice application of this formalism has been made, however, by Kubo (1964) and Jannusis, Streklas, and Vlachos (1981), to the description of electrons moving in a magnetic field.

\section{E. Second-quantization approach to inclusive reactions}

In Sec. IV.E we considered the $N$-body problem for a system of identical particles in the framework of second quantization. Here we approach the scattering problem via the route of reduction formulas and in-out fields (Goldberger and Watson, 1964). For the Hamiltonian
(4.71), dropping indices, we find the equation of motion for the Heisenberg interpolating field $\psi$ to be

$$
\left(i \frac{\partial}{\partial t}-\frac{\nabla^{2}}{2 m}\right) \psi=j
$$

where the current $j$ is

$$
j(x)=\int d^{3} x^{\prime} \psi^{+}\left(x^{\prime}\right) v\left(x-x^{\prime}\right) \psi\left(x^{\prime}\right) \psi(x) .
$$

In the sense of weak convergence, we have the limits

$$
\begin{aligned}
& \psi \rightarrow \psi_{\text {in }}, \quad t \rightarrow-\infty, \\
& \psi \rightarrow \psi_{\text {out }}, \quad t \rightarrow+\infty .
\end{aligned}
$$

The particle coordinates are defined by

$$
\psi(\underline{x}, t)=\int \frac{d^{3} p}{(2 \pi)^{3 / 2}} e^{i \underline{p} \cdot \underline{x}} a(\underline{p}, t),
$$

where $a_{\text {out, in }}(\underline{p}, t)=a_{\text {out, in }}(\underline{p}) \exp \left(-i p^{2} t / 2 m\right)$, with the $a$ 's obeying Eq. (4.63). The $a_{\text {out }}$ and $a_{\text {in }}$ coordinates destroy suitable out and in field quanta.

In order to integrate formally the equation of motion (5.18), we need the retarded Green's function

$$
\left\lceil i \frac{\partial}{\partial t}+\frac{\nabla^{2}}{2 m}\right) G_{R}(\underline{x}, t)=\delta(t)
$$

Fourier transforming $G$ as

$$
G_{R}(\underline{x}, t)=\int \frac{d \omega d^{3} p}{(2 \pi)^{4}} e^{-i \omega t+\underline{p} \cdot \underline{x}} G_{R}(\underline{p}, \omega),
$$

we find in momentum space

$$
\begin{aligned}
G_{R}(\underline{p}, \omega) & =\left(\omega-p^{2} / 2 m+i \epsilon\right)^{-1} \\
i G_{R}(\underline{x}, t) & =\int \frac{d^{3} \underline{p}}{(2 \pi)^{3}} e^{i \underline{p} \cdot \underline{x}-i p^{2} / 2 m t} \vartheta(t), \\
i G_{R}(\underline{x}, t) & =e^{+i t \nabla^{2} / 2 m} \delta(\underline{x}) \\
& =\frac{e^{i m \underline{x}^{2} / 2 t}}{[2 \pi t / m i]^{3 / 2}} .
\end{aligned}
$$

In terms of the in field, therefore, we have

$$
\begin{aligned}
\psi(\underline{x}, t)= & \psi_{\mathrm{in}}(\underline{x}, t) \\
& -i \int d^{3} x^{\prime} \int \frac{d^{3} p}{(2 \pi)^{3}} \int_{-\infty}^{t} d t^{\prime} j\left(\underline{x}^{\prime}, t^{\prime}\right) \\
& \times e^{i p_{1}\left(x-x^{\prime}\right)-i p^{2} / 2 m\left(t-t^{\prime}\right)} .
\end{aligned}
$$

The out field similarly involves the advanced Green's function. Using (in the sense of weak convergence)

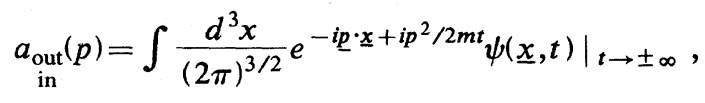

we easily derive 


$$
\begin{aligned}
& a_{\text {out }}(\underline{p})=a_{\text {in }}(\underline{p})-i j\left(\underline{p}, \omega=p^{2} / 2 m\right) /(2 \pi)^{3 / 2}, \\
& \left.j(\underline{p}, \omega) \equiv \int d^{3} x d t e^{i \omega t-i \underline{p} \cdot \underline{x}_{j}} j \underline{x}, t\right) .
\end{aligned}
$$

Now consider the reaction

$$
A+B \rightarrow n+X,
$$

where $X$ denotes a sum over all other possibilities and $N$ denotes one of the $\psi$ quanta. The outgoing number distribution

$$
\frac{d N_{\text {out }}}{d^{3} p_{n}}=\left\langle A B \text { in }\left|a_{\text {out }}^{+}\left(p_{n}\right) a_{\text {out }}\left(p_{n}\right)\right| A B \text { in }\right\rangle
$$

is, apart from the flux factor, the inclusive differential cross section for production of particle $n$.

To exhibit the connection with the Wigner distribution in its simplest form, we assume that $\mid A B$ in $\rangle$ contains no $n$ quanta. Then Eq. (5.32) is

$$
\int d^{4} x d^{4} x^{\prime} e^{-i p_{n} \cdot\left(x-x^{\prime}\right)}\left\langle A B \text { in }\left|j^{+}(x) j\left(x^{\prime}\right)\right| A B \text { in }\right\rangle,
$$

using four-vector notation. Changing variables to $R=1 / 2\left(X+X^{\prime}\right), r=x^{\prime}-x$, we find

$$
\begin{aligned}
\int d^{4} R \int d^{4} r e^{i p_{n} \cdot r} & \\
& \times\left\langle A B \text { in }\left|j^{+}\left(R-\frac{1}{2} r\right) j\left(R+\frac{1}{2} r\right)\right| A B \text { in }\right\rangle .
\end{aligned}
$$

Since $\mid A B$ in $\rangle$ is a normalized state, we cannot and do not want to use translation invariance to remove the $R$ dependence.

Comparison with Eq. (2.18) for the one-particle wavefunction case reveals a very similar structure. The cross section naturally involves currents, while the simplest theoretical object involves the fields.

Defining the second-quantized Wigner function as

$$
\begin{aligned}
F(p, R) \equiv \int & d^{4} r e^{i p \cdot r} \\
& \times\left\langle A B \text { in }\left|\psi^{+}\left(R-\frac{1}{2} r\right) \psi\left(R+\frac{1}{2} r\right)\right| A B \text { in }\right\rangle,
\end{aligned}
$$

an obvious generalization of Eq. (4.61) for $\rho=\mid A B$ in $\rangle\langle A B$ in $|$, we find that the corresponding function involving currents is

$$
\begin{aligned}
\widetilde{F}(p, R) \equiv \int & d^{4} r e^{i p \cdot r} \\
& \times\left\langle A B \text { in }\left|j^{+}\left(R-\frac{1}{2} r\right) j\left(R+\frac{1}{2} r\right)\right| A B \text { in }\right\rangle .
\end{aligned}
$$

We define $\widetilde{F}(p, q)$ by

$$
\begin{aligned}
\widetilde{F}(p, q)= & \int d^{4} R e^{-i q \cdot R} \widetilde{F}(p, R) \\
= & \int d^{4} x d^{4} x^{\prime} e^{i(p-q / 2) \cdot x^{\prime}} e^{-i(p+q / 2) \cdot x} D^{+}(x) \\
& \times\left\langle A B \text { in }\left|\psi^{+}(x) \psi\left(x^{\prime}\right)\right| A B \text { in }\right\rangle \mathbf{D}\left(x^{\prime}\right) .
\end{aligned}
$$

where $D(x) \psi(x) \equiv j(x)$. Integrating by parts, we can factor out free inverse propagators, giving

$$
\widetilde{\boldsymbol{F}}(p, q)=\boldsymbol{G}^{-1}\left(p+\frac{1}{2} q\right) \boldsymbol{F}(p, q) \boldsymbol{G}^{-1}\left(p-\frac{1}{2} q\right),
$$

where $F(p, q)$ is defined from Eq. (5.35) in analogy to the first line of Eq. (5.37).

The physical cross section (5.34) is determined by

$$
\frac{d N}{d^{3} p_{n}}=\widetilde{F}\left(p_{n}, q=0\right) \text {. }
$$

To find $\widetilde{F}$, we write the equation of motion for $F(p, R)$ using Eq. (5.18). Clearly this leads again to a hierarchy structure to be solved in some approximate way. For example, factorization of the fourfold operator leads directly to TDHF for fermions. Having found $F(p, q)$, one amputates external legs and takes the limit $q \rightarrow 0$ as in Eq. (5.39).

Our formalism is now set up in a form suitable for extension to the relativistic regime. Note that formally there is no problem in setting up the reduction formulas for outgoing composites.

Finally we note that, in the continuum limit, Eq. (5.34) is nothing but a generalized absorptive part. Hence the coupled hierarchy equations for the generalized Wigner functions constitute an approach to evaluating the absorptive parts of the theory.

\section{F. Relativistic formulation of the phase-space approach to scattering and production processes}

After this long essay we finally reach the starting point of the whole investigation-the formulation of a suitable unified transport theory approach to the description of inclusive multiparticle production processes in the ultrarelativistic domain. This technique was designed to avoid the naive oversimplification of perturbative approaches such as the multiperipheral model, which typically discard the extensive final-state interactions which play a dominant role in collective effects. In many ways this program was too ambitious in attempting to bypass the complex structural questions already posed by the nonrelativistic many-body problem. In addition, the special problems posed by QCD and quark confinement seemed to require too extensive an investment of effort in the absence of a clear understanding of the analogous nonrelativistic problems.

In order to express the basic ideas of this approach, we consider the production of spinless neutral particles (Carruthers and Zachariasen, 1976). Given an incoming normalized Heisenberg state $\Psi_{\text {in }}$, we define a covariant oneparticle distribution function $F(p, R)$ by

$F(p, R) \equiv \int d^{4} r e^{i p \cdot r}\left\langle\Psi_{\text {in }}\left|\varphi\left(R-\frac{1}{2} r\right) \varphi\left(R+\frac{1}{2} r\right)\right| \Psi_{\text {in }}\right\rangle$,

where $\varphi(x)$ is the usual Klein-Gordon field obeying the equation of motion 


$$
\left(\square+m^{2}\right) \varphi(x)=j(x),
$$

and where $j(x)$ is the current source depending on the detailed theory under consideration. $\square=\partial_{\mu} \partial^{\mu}$ is the Dalembertian, with a metric $(+---)$.

Since we wish to retain information about the spacetime localization, we use normalized states $\Psi_{\text {in }}$ so that the $R$ dependence does not cancel out of the matrix elements. In analogy to Eq. (5.32), we have

$$
\frac{d N}{d^{3} p}=\left\langle\Psi_{\text {in }}\left|a_{\text {out }}^{+}(p) a_{\text {out }}(p)\right| \Psi_{\text {in }}\right\rangle
$$

for the outgoing number distribution of the quanta of the field $\varphi$. Using standard reduction formula techniques leads to the formula

$$
\begin{aligned}
2 \omega \frac{d N}{d^{3} p}=\frac{1}{(2 \pi)^{3}} \int d^{4} x_{1} \int d^{4} x_{2} e^{i p \cdot\left(x_{2}-x_{1}\right)} \\
\times\left\langle\Psi_{\text {in }}\left|j\left(x_{1}\right) j\left(x_{2}\right)\right| \Psi_{\text {in }}\right\rangle .
\end{aligned}
$$

The right-hand side can be written as

$$
2 \omega \frac{d N}{d^{3} p}=\left[\frac{\left(p^{2}-\mu^{2}\right)^{2}}{(2 \pi)^{3}} \int d^{4} R F(p, R)\right]_{p^{2}=\mu^{2}}
$$

When $\Psi_{\text {in }}$ describes two colliding incident particles, $2 \omega d N / d^{3} p$ is the usual one-particle inclusive differential cross section apart from the incident flux factor.

As in the previous section, it is useful to define an auxiliary function in terms of the current,

$$
\widetilde{F}(p, R) \equiv \int d^{4} r e^{i p \cdot r}\left\langle\Psi_{\text {in }}\left|j\left(R-\frac{1}{2} r\right) j\left(R+\frac{1}{2} r\right)\right| \Psi_{\text {in }}\right\rangle .
$$

Defining Fourier tranforms as in Eq. (5.37) leads to

$$
\widetilde{F}(p, q)=\left[\left(p+\frac{1}{2} q\right)^{2}-\mu^{2}\right]\left[\left(p-\frac{1}{2} q\right)^{2}-\mu^{2}\right] F(p, q) \text {. }
$$

In this notation we can write Eq. (5.44) as

$$
2 \omega \frac{d N}{d^{3} p}=\left.\frac{1}{(2 \pi)^{3}} \widetilde{F}(p, q)\right|_{q=0} .
$$

An equation-of-motion approach paralleling the usual Boltzmann approach can be easily found for the function $F(p, R)$ by subtracting the two equations of motion

$$
\left(\square_{1}+\mu^{2}\right)\left\langle\varphi\left(x_{1}\right) \varphi\left(x_{2}\right)\right\rangle=\left\langle j\left(x_{1}\right) \varphi\left(x_{2}\right)\right\rangle
$$

and

$$
\left(\square_{2}^{2}+\mu^{2}\right)\left\langle\varphi\left(x_{1}\right) \varphi\left(x_{2}\right)\right\rangle=\left\langle\varphi\left(x_{1}\right) j\left(x_{2}\right)\right\rangle .
$$

Defining $R=\left(x_{1}+x_{2}\right) / 2$ and $r=x_{2}-x_{1}$, we note the identity

$$
\square_{2}^{2}-\square_{1}^{2}=2 \frac{\partial}{\partial R} \cdot \frac{\partial}{\partial r} .
$$

The basic equation is then

$$
\begin{aligned}
2 i p \cdot \frac{\partial}{\partial R} F(p, R)=\int d^{4} r e^{i p \cdot r} & \\
\times[ & \left\langle j\left(R-\frac{1}{2} r\right) \varphi\left(R+\frac{1}{2} r\right)\right\rangle \\
& \left.\quad-\left\langle\varphi\left(R-\frac{1}{2} r\right) j\left(R+\frac{1}{2} r\right)\right\rangle\right] .
\end{aligned}
$$

The operator $p \cdot \partial / \partial R=p_{0}\left(\partial / \partial t+\mathbf{p} / p_{0} \cdot \nabla\right)$ is the covariant analog of the usual $D / D t$ of ordinary hydrodynamics. The right-hand side is highly model dependent.

Ordinarily the interaction Lagrangian is such that the right-hand side of Eq. (5.50) cannot be expressed in terms of the one-particle distribution itself, but instead will require the introduction of higher-order distribution functions which will, in turn, generate still more complicated objects on the right-hand side of their equation of motion.

The simplest case-analogous to the simple harmonic oscillator driven by a $C$-number source-involves a Lorentz scalar source $V(x)$ coupled linearly to the meson field, i.e.,

$$
L_{\text {int }}=V(x) \varphi(x) .
$$

For this simple case, the current is $j(x)=V(x)$ and the formal solution to the equation of motion,

$$
\varphi(x)=\varphi_{\text {in }}(x)-\int d^{4} x^{\prime} \Delta\left(x-x^{\prime}\right) V\left(x^{\prime}\right)
$$

where $\Delta(x)$ is the free retarded Green's function, is an explicit solution.

Particle production from the incoming vacuum is now easily computed in terms of $\widetilde{F}$ as

$$
\widetilde{F}(p, q)=V\left(p+\frac{1}{2} q\right) V^{*}\left(p-\frac{1}{2} q\right),
$$

giving the usual number distribution:

$$
\frac{d N}{d^{3} p}=\frac{|V(p)|^{2}}{2 \omega(2 \pi)^{3}} \text {. }
$$

In order of complexity, the next most difficult problem is the pair model, with

$$
L_{\text {int }}=\frac{1}{2} V(x) \varphi^{2}(x) .
$$

This problem is basically just a relativistic Schrödinger problem. In momentum-space the equation of motion is

$$
\begin{aligned}
2 p \cdot q F(p, q)=\int \frac{d^{4} q^{\prime}}{(2 \pi)^{4}} V\left(q^{\prime}\right) & \\
\times & {\left[F\left(p+\frac{1}{2} q^{\prime}, q-q^{\prime}\right)\right.} \\
& \left.\quad-F\left(p-\frac{1}{2} q^{\prime}, q-q^{\prime}\right)\right] .
\end{aligned}
$$

Consider the solution of this equation in an iterative scheme, beginning with an incoming vacuum, having an unperturbed distribution

$$
F_{0}(p, q)=(2 \pi)^{3} \delta(q) \delta_{-}\left(p^{2}-\mu^{2}\right)
$$

appropriate to uniform space-time; $\delta_{-}$means that only the $p_{0}<0$ root of the $\delta$ function is to be taken.

To second order, we find 


$$
\begin{aligned}
F(p, q)= & (2 \pi)^{3} \delta_{-}\left(p^{2}-\mu^{2}\right) \delta(q)-2 \pi\left(\frac{\delta_{-}\left[\left(p+\frac{1}{2} q\right)^{2}-\mu^{2}\right]}{\left(p-\frac{1}{2} q\right)^{2}-\mu^{2}}+\frac{\delta_{-}\left[\left(p-\frac{1}{2} q\right)^{2}-\mu^{2}\right]}{\left(p+\frac{1}{2} q\right)^{2}-\mu^{2}}\right] \\
& \times\left(V(q)-\int \frac{d^{4} q^{\prime}}{(2 \pi)^{4}} \frac{V\left(q^{\prime}\right) V\left(q-q^{\prime}\right)}{\left[\left(p+p^{\prime}+\frac{1}{2} q\right)^{2}-\mu^{2}\right]}\right)+\frac{2 \pi}{\left[\left(p+\frac{1}{2} q\right)^{2}-\mu^{2}\right]\left[\left(p-\frac{1}{2} q\right)^{2}-\mu^{2}\right]} \\
& \times \int \frac{d^{4} p^{\prime}}{(2 \pi)^{4}} V\left(q^{\prime}\right) V\left(q-q^{\prime}\right) \delta_{-}\left[\left(p+p^{\prime}-\frac{1}{2} q\right)^{2}-\mu^{2}\right] .
\end{aligned}
$$

Inspection of these contributions (and higher-order terms) shows that Eq. (5.58) is the sum of the discontinuities of a scattering amplitude for mesons of initial and final momenta $p \pm \frac{1}{2} q$ off the potential $V$ (Fig. 10 ). $\widetilde{F}(p, q)$ corresponds to including discontinuities of internal lines only and amputating external legs. The lowest-order contribution to $\widetilde{F}$ is

$$
\begin{aligned}
\widetilde{F}(p, q)=2 \pi \int & \frac{d^{4} p^{\prime}}{(2 \pi)^{4}} V\left(p^{\prime}\right) V\left(q-p^{\prime}\right) \\
& \times \delta_{-}\left[\left(p+p^{\prime}-\frac{1}{2} q\right)^{2}-\mu^{2}\right],
\end{aligned}
$$

giving the number distribution of produced particles as

$$
\frac{d N}{d^{3} p}=\frac{1}{2 \omega(2 \pi)^{3}} \int d^{4} q|V(p+q)|^{2} \delta_{+}\left(q^{2}-\mu^{2}\right) \text {. }
$$

Generalization to higher-order inclusive cross sections and possible collective modes can be found in the cited reference (Carruthers and Zachariasen, 1976). However, few insights into the collision problem were yielded by this investigation.

For the purposes of the present article, the main point of the present section is to show that relativistic kinematics sees no barrier to the definition of appropriate phasespace distributions, cross sections, and equations of motion. In describing particles with spin, however, tedi-

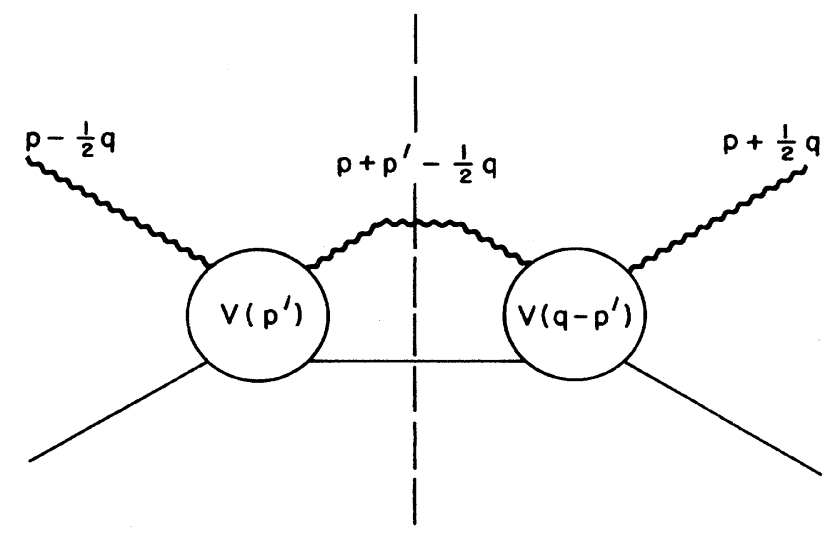

FIG. 10. Discontinuity (represented by the vertical dashed line) of the scattering amplitude for meson $p-\frac{1}{2} q$ to meson $p+\frac{1}{2} q$ in the presence of the potential $V$ (schematically indicated by the straight lines) in second-order perturbation theory. ous technical complications arise. For Dirac particles of spin $\frac{1}{2}$, a fully general treatment requires a $4 \times 4$ matrix phase-space density, for example (Hakim, 1978). At present the study of extended relativistic systems in QCD and their equations of state is a fully developed subject (Hakim, 1978, Shuryak, 1980). Still, the application to relativistic scattering is terra incognita. The formalism of this section is, however, especially suited for the production of nonconserved quanta, such as pions in nucleusnucleus collisions. For example, in the popular model of $\Delta N \pi$ coupling with the Lagrangian

$$
L_{\text {int }}=g(\bar{\Delta} N \pi+\text { H.C. }),
$$

the current is $j=g(\bar{\Delta} N+$ H.C. $)$, giving a structure to the right-hand side of Eq. (5.50) of the form

$$
\left\langle\Psi\left|\bar{\Delta} N \frac{1}{\square+\mu^{2}} \bar{N}\right| \Psi\right\rangle,
$$

which gives, for positive energy states, a covariant version of the $\Delta$-hole model. Naturally, the development of this formal structure into a full-blooded cross section requires extensive work. Nevertheless, the present theory provides all the ingredients for a satisfactory description of this and similar processes.

\section{ACKNOWLEDGMENTS}

We are indebted to Dr. E. Heller for useful conversations and remarks on the manuscript. This work was supported in part by the U.S. Department of Energy.

\section{REFERENCES}

Agarwal, G. S. and E. Wolf, 1968, Phys. Rev. 21, 180.

Ali, S. T., and E. Prugovečki, 1977a, J. Math. Phys. 18, 219.

Ali, S. T., and E. Prugovečki, 1977b, Int. J. Theor. Phys. 16, 689.

Baker, G. A., 1958, Phys. Rev. 109, 2198.

Baker, G. A., I. E. McCarthy, and C. E. Porter, 1960, Phys. Rev. 120, 254.

Balazs, N. L., 1980, Physica A 102, 236.

Balazs, N. L., and G. G. Zipfel, Jr., 1973, Ann. Phys. (N.Y) 77, 139.

Barut, A. O., 1957, Phys. Rev. D 108, 565.

Berry, M. V., 1977, Philos. Trans. R. Soc. London 287, 30. 
Berry, M. V., and N. L. Balazs, 1979, J. Phys. A 12, 625.

Berry, M. V., N. L. Balazs, M. Tabor, and A. Voros, 1979, Ann. Phys. (N.Y.) 122, 26.

Bezzerides, B., and D. F. DuBois, 1972, Ann. Phys. (N.Y.) 70, 10.

Bialynicki-Birula, I., 1977, Acta Phys. Austriaca Suppl. XVIII, p. 112.

Bodmer, A. R., and C. N. Panos, 1977, Phys. Rev. C 15, 1342.

Brittin, W. E., 1971, in Topics in Modern Physics-A Tribute to E. U. Condon, edited by W. E. Brittin and H. Odabasi (Colorado Associated Universities Press, Boulder), p. 45.

Brout, R., and P. Carruthers, 1968, Lectures on the ManyElectron Problem (Gordon and Breach, New York).

Brown, R. C., and E. J. Heller, 1982, "Classical Trajectory Approach to Photodissociation: The Wigner Method," UCLA preprint.

Callaway, D. J. E., L. Wilets, and Y. Yariv, 1979, Nucl. Phys. A 327, 250.

Carruthers, P., 1974, Ann. N. Y. Acad Sci. 229, 91.

Carruthers, P., 1982, in Proceedings of the 5th High Energy

Heavy Ion Study LBL, May 1981 (LBL, Berkeley), p. 499.

Carruthers, P., and Minh Duong-Van, 1973, Phys. Rev. D 8, 859.

Carruthers, P., and M. M. Nieto, 1965, Am. J. Phys. 33, 537.

Carruthers, P., and M. M. Nieto, 1968, Rev. Mod. Phys. 40, 411.

Carruthers, P., and F. Zachariasen, 1965, in Theories and Experiments in High Energy Physics, edited by B. Kursunoglu, A. Perlmutter, and S. Widmayer (Plenum, New York), p. 349.

Carruthers, P., and F. Zachariasen, 1974, in Particles and Fields, edited by Carl Carlson, AIP Conference Proceedings No. 23 (AIP, New York), p. 481.

Carruthers, P., and F. Zachariasen, 1976, Phys. Rev. D 13, 950.

Cohen, E. G. D., Ed., 1968, Fundamental Problems in Statistical Mechanics (North-Holland, Amsterdam), Vol. II.

Cohen, Leon, 1966, J. Math. Phys. 7, 781.

Cohen, L., and Y. I. Zaparovanny, 1980, J. Math. Phys. 21, 794.

Dahl, Jens Peter, 1981, "Dynamical equations for the Wigner

function," Technical University of Denmark preprint.

Davies, R. W., and K. T. R. Davies, 1975, Am. J. Phys. 89, 261.

de Boer, W. P. H., 1979, Proc. Kon. Ned. Akad. Wet., Ser. B 82 (4), 337, 353

de Boer, W. P. H., 1980, Proc. Kon. Ned. Akad. Wet., Ser. B 83 (1), 1,16

de Groot, S. R., and L. G. Suttorp, 1972, Foundations of Electrodynamics (North-Holland, Amsterdam).

Eu, B. C., 1971, J. Chem. Phys. 54, 559.

Eu, B. C., 1973, J. Chem. Phys. 58, 1352.

Eu, B. C., 1975, J. Chem. Phys. 63, 298.

Fetter, A. L., and J. D. Walecka, 1971, Quantum Theory of Many-Particle Systems (McGraw-Hill, New York).

Ginocchio, J., 1978, Phys. Rev. C 17, 195.

Glauber, R., 1959, in Lectures in Theoretical Physics, edited by W. E. Brittin and L. G. Dunham (Interscience, New York), Vol. I, p. 315.

Glauber, R. J., 1963, Phys. Rev. 131, 2766.

Goldberger, M. I., and K. M. Watson, 1964, Collision Theory (Wiley, New York).

Gottfried, K., 1966, Quantum Mechanics (Benjamin, New York).

Groenewald, H. J., 1946, Physica 12, 405.

Grossman, A., and P. Huguenin, 1978, Helv. Phys. Acta 51, 252.
Gyulassy, M., 1980, Relativistic Nuclear Collisions Theory, Report No. LBL-11040.

Hakim, R., 1978, Riv. Nuovo Cimento 1 (No. 6), 1.

Heller, Eric J., 1976, J. Chem. Phys. 65, 1289.

Heller, Eric J., 1977, J. Chem. Phys. 67, 3339.

Hoffman, D. K., D. J. Kouri, and Z. H. Top, 1979, J. Chem. Phys. 70, 4640.

Huang, K., 1963, Statistical Mechanics (Wiley, New York).

Huguenin, P., 1973, Z. Naturforsch., Teil B 28a, (H. 7), 1090.

Imre, K., E. Özizmir, M. Rosenbaum, and P. F. Zweifel, 1967, J. Math. Phys. (N.Y.) 8, 1097.

Irving, J. H., and R. W. Zwanzig, 1951, J. Chem. Phys. 19, 1173.

Jannussis, A., A. Streklas, and K. Vlachos, 1981, Physica A $107,587$.

Kadanoff, L. P., and G. Baym, 1962, Quantum Statistical Mechanics: Green's Function Methods in Equilibrium and Non-Equilibrium Problems (Benjamin, New York).

Kan, K.-K., and J. J. Griffin, 1977, Phys. Rev. C 15, 1126.

Kapusta, J. I., 1980, Phys. Rev. C 21, 1301.

Klimontovich, Iu. L., 1967, Statistical Theory of Non-

Equilibrium Processes in a Plasma (MIT, Cambridge, Mass.)

Köhler, H. S., and H. Flocard, 1979, Nucl. Phys. A 323, 189.

Kubo, R., 1964, J. Phys. Soc. Jpn. 19, 2127.

Laing, J. R., and K. F. Freed, 1975, Phys. Rev. Lett. 34, 849.

Landau, L. D., 1953, Izv. Akad. Nauk SSSR 17, 51.

Lax, M., 1968, Phys. Rev. 172, 350.

Lee, H.-W., and M. O. Scully, 1980, J. Chem. Phys. 73, 2238.

Madelung, E., 1926, Z. Phys. 40, 322.

Metropolis, N. R. Bivens, M. Storm, A. Turkevich, J. M. Miller, and G. Friedlander, 1958, Phys. Rev. 110, 185.

Miles, J. R. N., and J. S. Dahler, 1970, J. Chem. Phys. 52, 616.

Miller, W. H., 1974, Adv. Chem. Phys. 25, 69.

Mori, H., I. Oppenheim, and J. Ross, 1962, in Studies in Statistical Mechanics, edited by J. de Boer and G. E. Uhlenbeck (North-Holland, Amsterdam), Vol. I.

Mori, H., and J. Ross, 1958, Phys. Rev. 109, 1877.

Moyal, E., 1949, Proc. Cambridge Philos. Soc. 45, 99.

Newton, R. G., 1966, Scattering Theory of Waves and Particles (McGraw-Hill, New York).

Nix, J. R., 1979, in Progress in Particle and Nuclear Physics, edited by D. Wilkinson (Pergamon, Oxford and New York), Vol. II.

Nix, J. R., and D. Strottman, 1981, Phys. Rev. C 23, 2548.

O'Connell, R. F., and E. P. Wigner, 1981a, Phys. Lett. A 85, 121.

O'Connell, R. F., and E. P. Wigner, 1981b, Phys. Lett. A 83, 145.

Prigogine, I., 1959, Non-Equilibrium Statistical Mechanics (Interscience, New York).

Prugovečki, E., 1978a, Physica A 91, 202.

Prugovečki, E., 1978b, Ann. Phys. (N.Y.) 110, 102.

Prugovečki, E., 1978c, Physica A 91, 229.

Remler, E. A., 1975, Ann. Phys. 95, 455.

Remler, E. A., 1981, Ann. Phys. 136, 293.

Remler, E. A., and A. P. Sathe, 1975, Ann. Phys. 91, 295.

Remler, E. A., and A. P. Sathe, 1978, Phys. Rev. C 18, 2293.

Ross, J., and J. Kirkwood, 1954, J. Chem. Phys. 22, 1094.

Schiff, L. I., Ed., 1968, Quantum Mechanics (McGraw-Hill, New York).

Schweber, S. S., 1961, An Introduction to Relativistic Quantum Field Theory (Row, Peterson, Evanston).

Shlomo, S., and M. Prakash, 1981, Nucl. Phys. A 357, 157.

Shuryak, E. V., 1980, Phys. Rep. 61, 71. 
Snider, R. F., 1960, J. Chem. Phys. 32, 1051.

Snider, R. F., and B. C. Sanctuary, 1971, J. Chem. Phys. 35, 1555.

Stelte, N., and R. Weiner, 1981, Phys. Lett. B 103, 275.

Takabayasi, T., 1954, Prog. Theor. Phys. 11, 341.

Thies, M., 1979, Ann. Phys. 123, 411.

Tolman, R. C., 1938, The Principles of Statistical Mechanics (Oxford University, London).

Uehling, E. A., and G. E. Uhlenbeck, 1933, Phys. Rev. 43, 552.

Uhlhorn, U., 1976, Ark. Fys. 11, 87.

von Neumann, J., 1955, Mathematical Foundation of Quantum Mechanics (Princeton University, Princeton).
Weidenmüller, H. A., 1980, in Progress in Particle Physics, edited by D. Wilkinson (Pergamon, Oxford and New York), Vol. III.

Weiner, R. M., 1978, Phys. Rev. D 13, 1363.

Weyl, H., 1927, Z. Phys. 46, 1.

Wigner, E. P., 1932, Phys. Rev. 40, 749.

Wigner, E. P., 1971, in Perspectives in Modern Physics, edited by W. Yourgrau and A. VanderMere (MIT, Cambridge, Mass.), p. 25.

Wilets, L., E. M. Henley, M. Kraft, and A. D. MacKellar, 1977, Nucl. Phys. A 282, 341.

Yariv, Y., and Z. Fraenkel, 1979, Phys. Rev. C 20, 222. 